\title{
The Development and Characterization of a Ball Mill for Mechanical Alloying
}

\author{
By
}

Tawfik Taher Ajaal

A thesis submitted to the Department of Materials and Metallurgical Engineering in conformity with the department requirements for the degree of Master of Science (Engineering)

\author{
Queen's University \\ Kingston, Ontario, Canada
}

December, 1999

Copyright @ Tawfik Ajaal, 1999 
National Library of Canada

Acquisitions and Bibliographic Services

395 Wellington Street Ottawa ON K1A ONA Canada
Bibliothèque nationale du Canada

Acquisitions et services bibliographiques

395, rue Wellington Ottawa ON K1A ON4

Canada
The author has granted a nonexclusive licence allowing the National Library of Canada to reproduce, loan, distribute or sell copies of this thesis in microform, paper or electronic formats.
L'auteur a accordé une licence non exclusive permettant à la Bibliothèque nationale du Canada de reproduire, prêter, distribuer ou vendre des copies de cette thèse sous la forme de microfiche/film, de reproduction sur papier ou sur format électronique.

L'auteur conserve la propriété du droit d'auteur qui protège cette thèse. $\mathrm{Ni}$ la thèse ni des extraits substantiels de celle-ci ne doivent être imprimés ou autrement reproduits sans son autorisation. 


\section{Abstract}

Ball milling has been used to produce fine particles from coarse feed for an extended period. However, in the traditional ball mill, the energy exchange between the tumbling balls themselves and the feedstock tends to be chaotic in some circumstances. If the trajectory of the ball can be controlled at will, then, a far more selective comminution process can be devised, which offers considerable advantages in reducing grinding costs by conserving momentum for feed stock fracture rather than grinding time and ball consumption. To overcome the limitations of the conventional ball mill, an improved ball mill has been constructed. The new ball mill is a hollow cylinder made from a hard paramagnetic material, with permanent magnets mounted outside the chamber. The strength of the magnets and the fact that the magnets can be moved around the chamber along an arc centered on the axis of the rotation of the chamber can be used to control the ball movement. The magnet's field is used to control the movement of the steel balls when the chamber is rotated. By controlling the ball movement inside the chamber using an external magnetic field, the limitations of the conventional ball mill may be reduced.

In commercially available ball mills, the movement of the balls is chaotic, driven by the accidental collision between the balls themselves or with the walls of the chamber. The distribution of milling energies is quite broad. In the improved ball mill, the balls can move according to a pre-set pattern, which results in a well-defined value of the milling energy. The ball movement pattern is controlled externally. The designing, building and testing of an improved ball mill is the subject of this study. 
Under the same operating conditions, the improved ball mill required less energy and produced finer and more homogeneous particles as compared with conventional ball milling. The energy consumed in the particle size reduction is calculated for the both modes of operation of the improved and the conventional ball mill. It is found that the improved ball mill saves $40 \%$ of the energy consumed by the conventional ball mill to produce an assigned particle size. The improved ball mill reduces the grinding time to the half of that of the conventional ball mill for an assigned particle size. The optimum condition of operating this particular improved ball mill $(3.5 \mathrm{~cm}$ length and $5 \mathrm{~cm}$ diameter) was determined to be 350 grams of media weight, the diameter of the steel ball is $1.25 \mathrm{~cm}, 87 \%$ of the theoretical critical speed and 50 grams of sample weight. 


\section{Acknowledgements}

I would like to thank Dr. R.W. Smith and Dr. T. Yen for supervising this project. Also, I would like to thank the technicians and the staff of the Materials and Metallurgical Engineering Department for their help and cooperation.

Many thanks go to Darrel Dietrich at the workshop for his valuable help on constructing the device. Also, I would like to express my gratitude to my friend Mohamed Hassan for the assistance in the computer work whenever necessary.

Finally, I would like to acknowledge the Libyan Government and the School of Graduate Studies at Queen's University for their tinancial support. 
Abstract ii

Acknowledgements iv

Table of Contents $\quad$ v

List of Figures viii

List of Tables $\quad x$

I- Introduction 1

1-1 Scope of the Study

1-2 Background 1

1-3 The Improved Ball Mill 2

1-4 Objectives $\quad 2$

1-5 The Technical Field of Study 4

II- Literature Review 5

2-1 Principles of Milling 5

2-2 Milling Processes 9

2-2-1 Microforging 9

2-2-2 Fracture 9

2-2-3 Agglomeration 9

2-3 Mechanism of Milling 9

2-4 Objective of Milling 10

2-4-1 Impact 11

2-4-2 Attrition II

2-4-3 Shear 12

2-4-4 Compression $\quad 12$

2-5 Action between Grinding Media and Particles $\quad 12$

2-5-1 Conventional Ball Mill 12

2-5-2 Improved Ball Mill 12

2-6 Milling Equipment $\quad 12$

2-6-1 Tumbler Ball Mills 13

2-6-2 Vibrating Ball Mills $\quad 13$

2-6-3 Attrition Mills 15

2-7 Conventional Ball Mill Specifications 17

2-8 Mode of Operation 21

2-8-1 Conventional Ball Mill 21

2-9 Kinetic Energy in Milling Devices $\quad 24$ 
2-10 Conversion and Transfer of Energy during Milling 25

2-11 Energy Consumed by Material 26

III- Design of the Improved Ball Mill 30

3-1 Concept of the Milling Device 30

3-2 Description of the Device 33

3-3 Design Description 33

3-3-1 Shell 33

3-3-2 Size 33

3-3-3 Magnet Holder $\quad 33$

3-3-4 Magnets 33

3-3-5 Magnets' Specifications $\quad 34$

3-4 Ball Mill Test Design 34

3-5 Improved Ball Mill Design 34

3-6 Configuration of the Magnetic Field 46

3-6-1 Field of Magnet 46

3-6-2 Force on a Magnet in a Magnetic Field 48

IV-Testing of the Improved Ball Mill 51

4-1 Mode of Operations

4-1-1 Mode A

4-1-2 Mode B

4-1-3 Mode C $\quad 52$

4-1-4 Mode D

4-1-5 Mode E $\quad 52$

4-2 Experimental Procedures and Results 54

4-2-1 Critical Speed $\quad 54$

4-2-2 Effect of Rotational Speed $\quad 55$

4-2-2-1 Conventional Ball Mill $\quad 55$

4-2-2-1 Improved Ball Mill 56

4-2-3 Particle Size Reduction $\quad 58$

4-2-3-1 Expressions of Size Reduction $\quad 59$

4-2-4 Effect of Grinding Media $\quad 62$

4-2-4-1 350g Media and 80\% of the Critical Speed 62

4-2-5 Ball Mill Grindability Test 63

4-2-5-1 Procedure 63

4-2-4-2 Calculation of the Bond Work Index 65

4-2-4-3 Bond Mill Grindability Test 66

4-2-6 Time/ Particle Size Relationship and Effect of Magnet 67

4-2-6-1 Example of Calculation $\quad 68$

4-2-7 Energy Consumed on the Particle Size Reduction 70

4-2-8 Particle Size / Energy 
4-2-9 Optimum Condition of Operation

4-2-9-3 Effect of Rotational Speed

4-2-10 Discussion

4-2-11 Physical Properties of the Grinding Material

4-2-11-1 Conventional Ball Mill

V-Conclusions

VI- Recommendations for Future Work

VII- References

VIII- Appendix 


\section{List of Figures}

Figure 1 Model of impact event at a time of maximum impacting force.................6

Figure 2 Process of trapping an incremental volume of powder between two balls........8

Figure 3 Tumbling ball mill......................................................14

Figure 4 Attrition ball mill ...................................................... 16

Figure 5 Path, forces, and zones of typical ball in a ball mill..........................20

Figure 6 Types of motion of balls in a planetary mill.................................23

Figure 7 Ball trajectories generated by different intensity and geometry

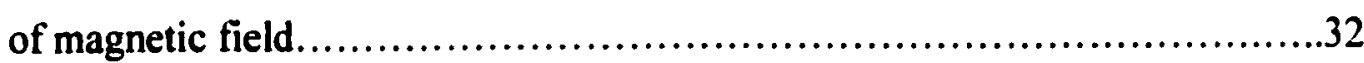

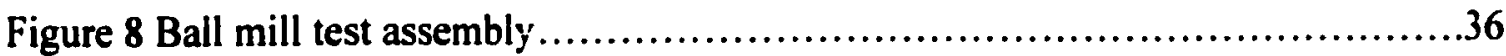

Figure 9 Ball mill test assembly showing cover removed............................37

Figure 10 Exploded view of the test ball mill....................................38

Figure 11 Pictorial view of improved ball mill............................................

Figure 12 Exploded view of improved ball mill....................................40

Figure 13 Pictorial view of improved ball mill showing full $12.5 \mathrm{~cm}$ length.............41

Figure 14 Pictorial view of improved ball mill showing reduced $5 \mathrm{~cm}$

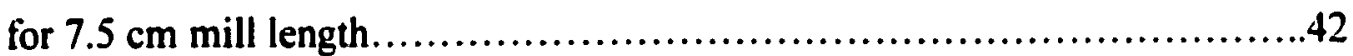

Figure 15 Pictorial view of improved ball mill showing reduced $10 \mathrm{~cm}$

for $2.5 \mathrm{~cm}$ mill length.....................................................

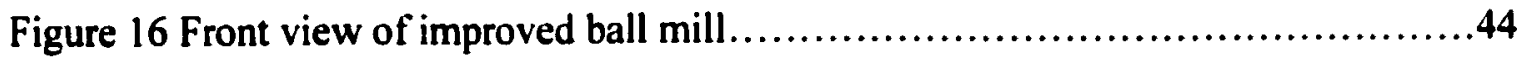

Figure 17 Plan view of improved ball mill...........................................45

Figure 18 Field produced at any point $\mathrm{P}$ by presence of bar magnet NS...............50

Figure 19 Modes of operation of the improved ball mill................................53

Figure 20 Effect of rotational speed on conventional ball mill efficiency.................56

Figure 21 Effect of rotational speed on the efficiency of the improved ball mill............57

Figure 22 Comparison between the improved and conventional mode of operation at $87 \%$ of the C.S. in terms of grinding ability under the same conditions ......57

Figure 23 Particle size reduction at $87 \%$ of the critical speed, 350 grams of media, and 50 grams of sample for the both modes of operation. 
Figure 24 Particle size reductions for both modes of operations at 30 minutes, $350 \mathrm{~g}$ media, and $87 \%$ of the critical speed...............................62

Figure 25 Effect of media loading on the grinding process for both modes................63

Figure 26 Effect of magnet on the particle size reduction at $350 \mathrm{~g}$ media,

$80 \%$ of the critical speed. and $50 \mathrm{~g}$ sample................................69

Figure 27 Energy consumed on the particle size reduction at $350 \mathrm{~g}$ of media loading,

$87 \%$ of the C.S. and $50 \mathrm{~g}$ of the sample weight for both modes of operation.....71

Figure 28 Comparison of the energy consumed on the particle size reduction of an improved and conventional ball mill at $750 \mathrm{~g}$ media, $87 \%$ of the critical speed, and 50g sample.................................................. 72

Figure 29 Effect of weight of sample on the grinding ability...........................74

Figure 30 Effect of media loading on the grinding ability ..............................75

Figure 31 Effect of rotational speed on the grinding ability...........................76 


\section{List of Tables}

Table 1 Rotational speed effect on the grinding process for a conventional ball mill......55

Table 2 Effect of rotational speed on the efficiency of the advanced ball mill..............56

Table 3 Particle size reduction in the advanced and conventional ball mill.................61

Table 4 Typical calculations in the Bond ball mill circuit grindability test.................66

Table 5 Calculation of the energy consumed on particle size reduction

of $50 \mathrm{~g}$ sample at $350 \mathrm{~g}$ media, and $89 \%$ of the critical speed.....................69

Table 6 Energy consumed in particle size reduction.................................71

Table 7 Illustration of the energy consumed on particle size reduction at $750 \mathrm{~g}$ media, and $87 \%$ of the critical speed................................72

Table 8 Effect of the sample weight on the grinding ability .............................73

Table 9 Effect of the media loading in the improved ball mill and its effect on the particle size reduction......................................74

Table 10 Effect of rotational speed on the grinding ability in the improved

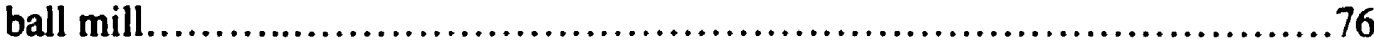




\section{I-Introduction}

\section{1-1 Scope of the Study}

For many years, the Department has been involved in the production and characterization of metastable and novel materials using quenching techniques. Interest is now moving to the use of mechanical alloying in order to produce fine-grained (nanocrystalline) materials. This project was conceived in order to develop an improved ball mill to be used ultimately in mechanical alloying. The thesis reports the work concerned with the development of the device and its characterization using a commercial ore, limestone.

\section{1-2 Background}

This project concerns ball milling. More particularly, it concerns the development of an improved ball mill for use in grinding and mechanical alloying. Ball mills and attritors have been used for many years to produce fine powders. In ball mills, the energy input to the powder charge is provided by the rotation of the mill, so that the hard balls within the mill are tumbled with, or onto, the charge in the mill. In attritors, metal arms are used to stir the ball charge. Using attritor mills, hardened steel balls are caused to impact vertically upon the powder charge. Local overheating of the particles can occur. This local overheating is difficult to remove. Also, the mixing of the particles is very low ${ }^{[1]}$. In the rotating mills, the steel balis roll along a circular arc on the inner wall of the mill chamber, and the powder charge is spread on the inner surface of the chamber. This ensures that the heat generated within the chamber is removed by conduction through the cylindrical wall, and there is a good mixing of the powder particles ${ }^{[2 !}$. However, the 
rotating mills are not able to provide the impact energy of the balls that is achieved in the attritor mills.

\section{1-3 The Improved Ball Mill}

In the case of a conventional ball mill operating at low speed, the balls move along the mill inner surface to a point where the component of gravity exceeds the centrifugal force and the balls fall from the mill shell in a parabolic path until they hit the mill shell. At higher speed, the balls begin to centrifuge but finally tumble in a cascading motion. For the case of the improved bail mill, at least one magnet is introduced to control the operation of the ball mill. By introducing magnet(s), new controllable ball trajectories are created, the exact nature of which depend on the position(s) of the magnet(s).

To create a cataracting action and very little cascading of the balls, the mill operates at an optimum speed. In the case of the improved ball mill, the magnet(s) create a magnetic field, which exerts a radial force on each ball. This affects the ball trajectories which as a result are controllable and are more uniform. The balls which are falling can hit balls at the bottom depending on the ball loading and the rotational speed. Consequently strong impact and shearing forces are created between the colliding masses. The grinding process appears to be controlled by impact. The flying path of the balls is controllable and reproducible.

\section{1-4 Objective}

The objective of the present study is to provide an improved ball mill in which the impact energy of the attritor mill can be achieved while the cooling and powder mixing of the rotating mill are maintained. Two models have been designed in this study, an improved ball mill test design, "Model I", and an improved ball mill design, "Model 2". 
The testing model, "Model l" is a simple design, with a clear plastic end for monitoring the ball movements while adjusting the magnet(s) positions around the mill. Pre-set patterns of operations are determined for each mode of operations. In general, by adjusting the magnetic field around the mill, different modes of operation may be experienced. In the improved ball mill design, "Model 2" different volumes of the mill can be obtained through an adjustable end plate. Also, the environment of milling is controllable in this design from vacuum to several hundred KPa over- pressures. Model 2 of the improved ball is not built and tested at the present study due to some technical difficulties in constructing it. Thus the objective of this study is designing, building and testing the test model "Model l"of the improved ball mill in which the energy of the milling process is variable and controllable.

The prototype model, "Model l" of the improved ball has been tested in terms of the particle size reduction and the energy consumed in the grinding process. A commercial ore, limestone, with a particle size all finer than $1200 \mu \mathrm{m}$, has been used in these tests. The results are compared with the results of a conventional ball mill under the same conditions and parameters, as illustrated in the Appendix. The energy consumed in the grinding process is calculated for the both modes of operation, and it is found to be less by $40 \%$ when using the improved mode of operation. The test model of the improved ball mill reduces the grinding time to the half of that of the conventional ball mill for an assigned particle size. Only one mode of operation of the improved ball mill is used, the high-energy mode, case E, Figure 19. Effects of the rotational speed, the weight of media, the weight of sample, and the effect of the magnetic force have been determined. The optimum condition of operating this improved ball mill in terms of the rotational 
speed, the media loading and the weight of sample is found to be $87 \%$ of the theoretical critical speed, 350 grams media loading and 50 grams weight of sample.

\section{1-5 The Technical Field of the Study}

The technical field of this study is summarized as follow: -

i) The improved ball mill provides the impact energy of the attritor mill and the cooling and the powder mixing of the rotating mill.

ii) Reduces the time of the grinding process and as a result the energy consumed in the particle size reduction reduces.

iii) Employs a magnetic field to control the ball motion in order to reduce the cost of efficient grinding and the mill wear.

vi) Provides a homogenous product with a narrow distribution of the particle size. 


\section{II-Literature Review}

\section{2-1 Principles of Milling}

The phenomenon of milling is typified in dry and wet milling operations that use colliding milling media such as rotating mills or attritor mills. The number of particles caught between two colliding balls is variable ranging from one particle to several thousand particles. It is dependent on the particle size and the density of the balls used in the milling operation. Also, there are other factors affecting the trapped particles between the collided balls such as the ball diameter relative to the particle diameter, and the amount of powder particles used ${ }^{[4]}$. Figure 1 shows the moment of the collision of two balls trapping an amount of particles between them. The trapped volume consists of particles within the region $B B^{\circ}-B^{\prime}$ and embraces many particles. Outside this region, the density of the powder particles decreases to the density of the loose powder in the mill. Figure 2 shows how particles are trapped between impacting balls. If the impacting force is sufficient, the trapped particles are compacted to form agglomerate as shown in Figure 2, d. If impact bonding between particles occurs and is sufficient, the agglomerate does not break down after the impact force is released. There is also a binding action between the particles and the surfaces of the balls. If the force applied on the particles is sufficient, the particles could be fractured or compacted within a zone of a diameter $\boldsymbol{X}_{\boldsymbol{c}}$ and thickness $t$ in the range $B B^{\prime}-B B^{\prime}$, Figure 1 . Theoretically, the zone of compaction is the region $A A^{\circ}-A A^{*}$. This compacted volume consists of some of the original particles and fragments of the others. The density of this trapped volume of particles varies from a maximum at the point of first contact between the two balls $C C^{\prime}$ to the density of the loose powder agitated outside the two $\boldsymbol{A A}^{\wedge}$ boundaries Figure ${ }^{\left[{ }^{[j]}\right.}$. 


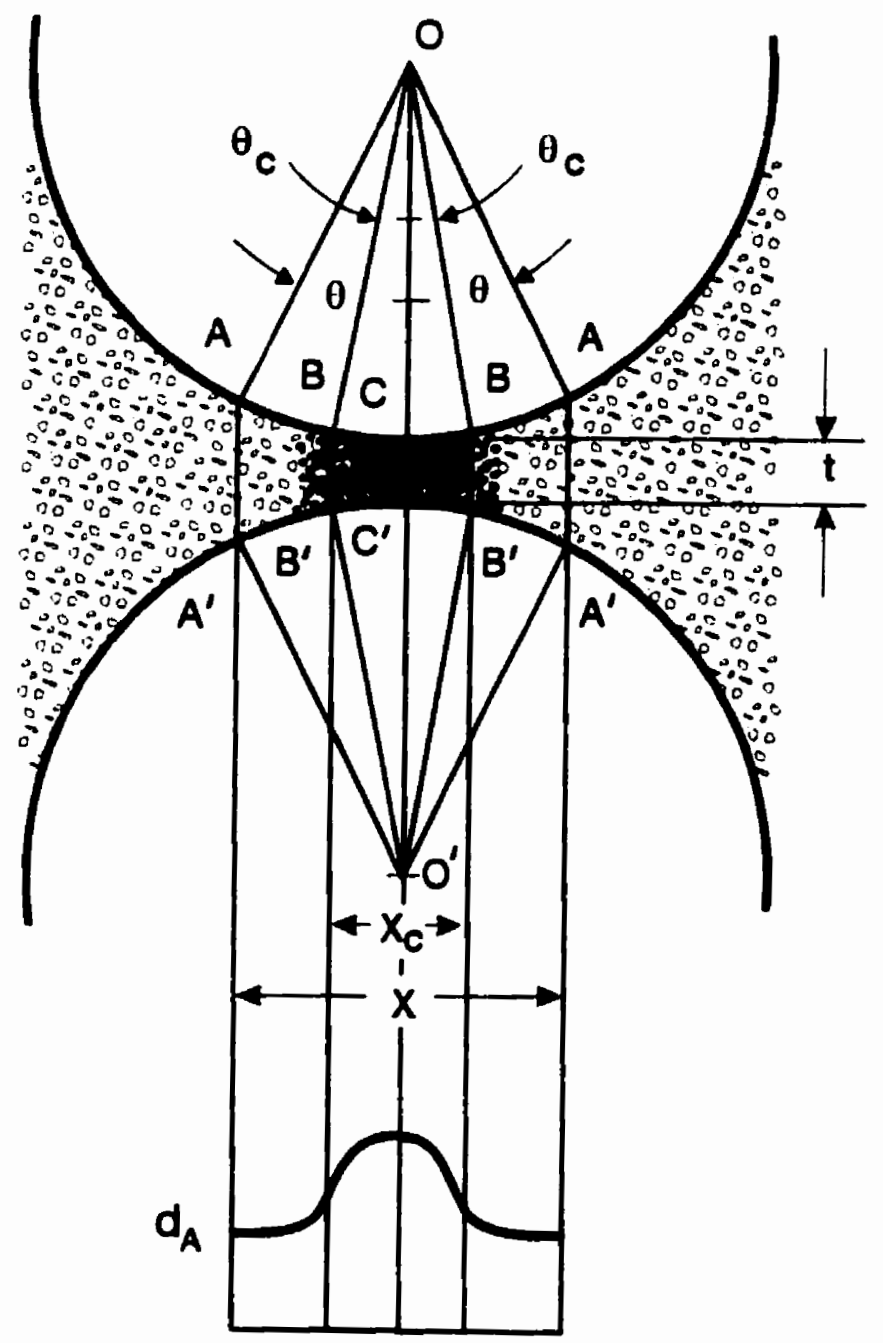

Figure 1 Model of impact event at a time of maximum impacting force. [ref.5] 
As the balls are decelerated during the impact, a radial displacement of powder particles occurs in the direction of least resistance to the particle flow, Figure 1 . The resistance to the displacement is due to the friction between the particles and the ball surfaces ${ }^{[6]}$. Such displacement is dependent on the surface roughness of both the particle and ball, and the degree of binding or cold welding between the agglomerated particles and the bail surfaces. The first stage of compaction begins with a powder mass that is characterized by a relatively large space between particles compared to the particle size. The finished product is a mass of powder with comparatively small pores. This stage of compaction consists of the rearrangement and restocking of the particles. During this stage the particles slide past one another producing some fines, which partially fill the void spaces. The second stage of compaction involves the elastic and plastic deformation of particles. Cold welding may also occur between the particles. Plastic deformation and cold welding have major effects in this stage of processing, depending on the ductility of the materials and the milling environment. The third stage of compaction involves particle fracture, which results in further deformation of the particles. The density of the powder bed reaches a maximum value at the zone $C^{\prime}$, Figure 1 . The strength of the compact formed depends on the strength of the bonds between particles, which in turn depends on factors such as the surface cleanliness, the temperature, and the impact force. The size of the impact depends on the above factors, as well as on the particle shape, the surface roughness of the balls and the particles, the size of the balls and of the particles. Particle-to-particle bond strength depends on the type of material being milled ${ }^{[7 \cdot 9 !}$. 
(a) through (c) Trapping and compaction of particles. (d) Agglomeration.

(e) Release of egglomerate by elastic energy.

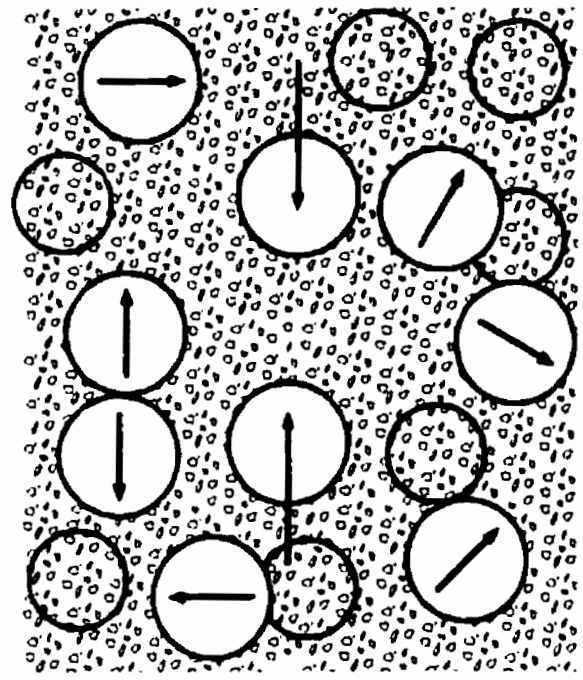

(a)

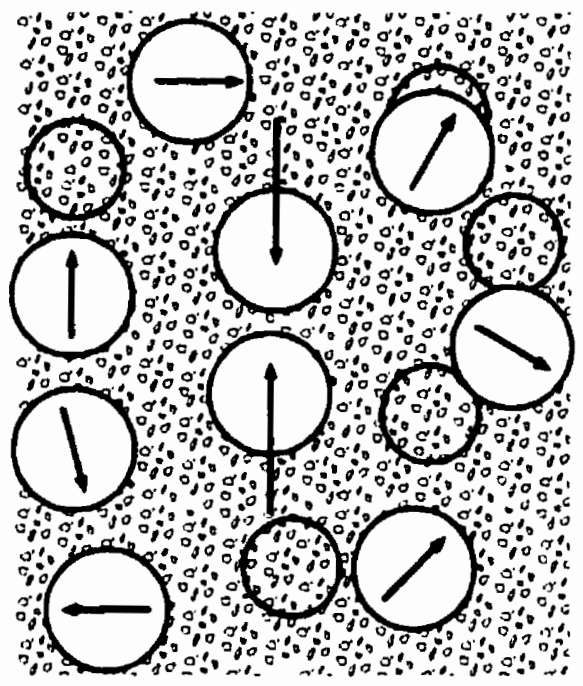

(b)

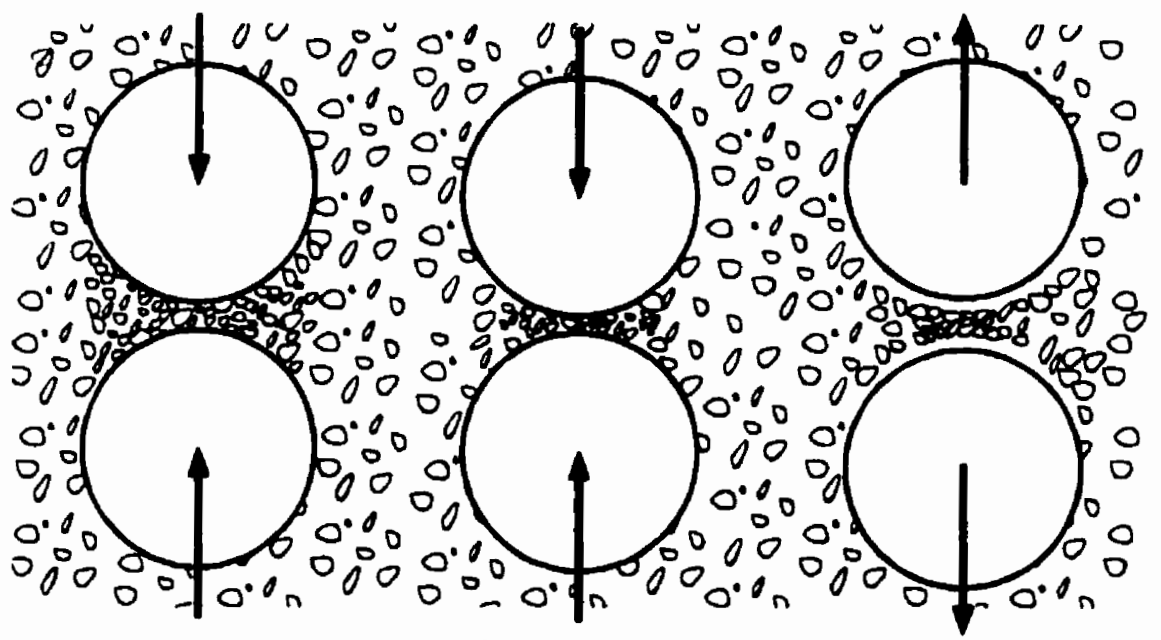

(c)

(d)

(e)

Figure 2 Process of trapping an incremental volume of powder between two balls.[ref.5] 


\section{2-2 Milling Processes}

The changes in powder particle morphology that occur during the milling of a material powder are produced by the following processes:

2-2-1 Microforging: The initial and predominant process during milling is the compression shape forming of ductile material particles by impact from the milling medium, (grinding balls, rods). Individual particles, or a cluster of particles. are impacted repeatedly by the milling medium so that they deform with little or no net change in individual mass $^{(8-10)}$.

2-2-2 Fractures: After a period of milling, individual particles deform to the extent that cracks initiate, propagate, and ultimately fracture the particles. Cracks, defects, and inclusions in particles facilitate fracturing. Particles formed from irregular or spongy particles contain fissures and cracks that facilitate compression, fatigue failure, and fragmentation, compared to the smooth, relatively nonporous spheroidal particles formed by atomization ${ }^{88-10]}$.

2-2-3 Agglomeration of particles may occur by welding, mechanical interlocking of spongy or rough surfaces, or autohesion. Autohesion is the molecular interaction of particles among themselves, characterized by van der Waals forces. Deagglomeration is

a process that breaks up agglomerates formed by autohesion without further disintegration of the individual powder particles ${ }^{[8-10]}$.

\section{2-3 Mechanism of Milling}

The milling mechanism consists of an initial stage of microforging. In this stage the particles deform by fracture and cold welding. As the particles become deformed, they 
enter a secondary stage caused by fatigue failure and fragmentation. Fragments generated may continue to the reduced in size by alternate microforging and fracture. When fracture dominates, particles with flake-like shape continue to become smaller. As these flakes become finer, the coupling forces tend to become greater and agglomerates become stronger. When the milling forces that deagglomerate the particles reach equilibrium with the coupling forces, a fixed agglomerate particle size results. The secondary stage of this mechanism involves a closed loop process of fracture, microforging, and agglomeration by welding. Constant kneading, fracturing, and rewelding produces a composite particle that consists of fragments of original and composite particles. The degree of refinement and blending of the composite structure is a function of the milling time ${ }^{[5.6]}$. After a period of time, cold welding and fracturing of the particles attain a steady state of equilibrium. The average particle size that can be obtained at this stage depends on the relative ease with which the agglomerates can be formed by welding, fatigue, fracture, and resistance of particles to the deformation. When a large number of fine particles are produced, the degree of refinement is very high. If the particles are rough, spongy, or have their surfaces covered with asperities, microforging may be accommodated by agglomeration $^{[8,9.11 !}$.

\section{2-4 Objective of Milling}

Increasing interest in material powder with particle sizes that are finer than the particie sizes of powders produced by atomization has reactivated interest in milling, particularly in solid-state alloying. Research conducted on the ball milling of materials is primarily proprietary and empirical. In most cases, the objective of milling is particle size reduction. The milling of material powder include the following objectives: 
- Particle size reduction.

- Particle size growth.

- Shape change.

- Agglomeration.

- Solid-state alloying (mechanical alloying).

- Solid-state blending (incomplete alloying).

- Modifying, changing, or altering properties of a material, (density, flowability, or work hardening).

- Mixing or blending of two or more materials or mixed phases.

The specific effect that milling will have on a powder depends on the physical and mechanical properties of the powder, and the environment in which the operation is conducted.

The selection of any milling process is based on the result desired of the milling operation, the behavior of the powder under milling conditions, the characteristics of the powder, and the physical and mechanical properties of the material.

During milling, four types of forces act on the material particles: impact. attrition. shear, and compression ${ }^{[7,12-16]}$.

2-4-1 Impact is the instantaneous striking of one object by another. Both objectives may be moving or one may be stationary ${ }^{[12,16]}$.

2-4-2 Attrition is the productions of wear debris or particles created by the rubbing action between two bodies ${ }^{[12,16]}$. 
2-4-3 Shear consists of cutting or cleaving of particle and usually is combined with other types of force. Shear contributes to fracturing by breaking particles into individual pieces with a minimum of fines ${ }^{[2,16]}$.

2-4-4 Compression is the application of compressive forces to a body (crushing or squeezing of particle material) ${ }^{[12.16\}}$.

\section{2-5 Actions between Grinding Media and Particles}

2-5-1 Conventional Ball mill: The grinding media, which are steel balls in this case, tumble over each other in the bed. Little cataracting action and more attrition action do the grinding process, the grinding process seems to be predominated by abrasion. The particles are rubbed between the bodies of the grinding media, and the mill wall. Most of the ground product in this type of mill is obtained at the expense of the small particles, due to the attrition action.

2-5-2 Improved Ball mill: In the improved ball mill, the steel balls are lifted by the magnetic force applied to the mill to a certain position. As the mill rotates the balls fall down on top of the other balls in the bed or on the wall mill striking number of particles between them. The grinding process is dominated by an impact action. Most of the ground product is obtained at the expense of the coarser particles because of the impact action.

\section{2-6 Milling Equipment}

A great variety of mills are available at the present time, differing in their type, size, design, and performance. The milling machine applies mechanical stresses to the 
individual particles in a powder mass to initiate fracture with a minimum of energy. The motion of milling medium and charge varies between types of mills with respect to: movement and trajectories of individual balls, movement of the mass of the balls, the degree of energy applied to impact, shear, attrition, and compression forces acting on powder particles, the optimum grinding medium charge in terms of fraction of mill volume occupied. Above the optimum, the tumbling action begins to reduce, until at $100 \%$ filling, the tumbling action is zero. The optimum filling depends on the type of the ball mill and grinding elements employed but is usually about $40 \%$ of the mill volume. Also the weight of the sample and the rotational speed affects the grinding process ${ }^{16.9 !}$.

\section{2-6-1 Tumbler Ball Mills}

In the tumbler mill, the motion of the balls depends on the rotation speed of the chamber walls. Balls may roll, or cascade down the suriace of the charge in a series of parallel layers or they may be projected, or cataract into space, falling on the balls and particles at the toe of the charge below, Figure 3. This mechanism is a function of the mill configuration such as the type and volume of the mill, the loading of the grinding medium and its size, the weight of sample, and the grinding ball density ${ }^{[6,9,10]}$.

\section{2-6-2 Vibratine Ball Mills}

Vibrating ball mills are high frequency, short stroke ball mills that impart the impact energy many more times per minute than the conventional ball mills. Therefore, they are ideally suited for fine grinding, reducing materials from coarse particles to finer ones. Most vibrating ball mills are constructed so that no force is imparted to the foundation; most of the force generated is directed at the material to be ground. They require no special foundation support and are very efficient ${ }^{[9,14]}$. 
HARDINGE MILL

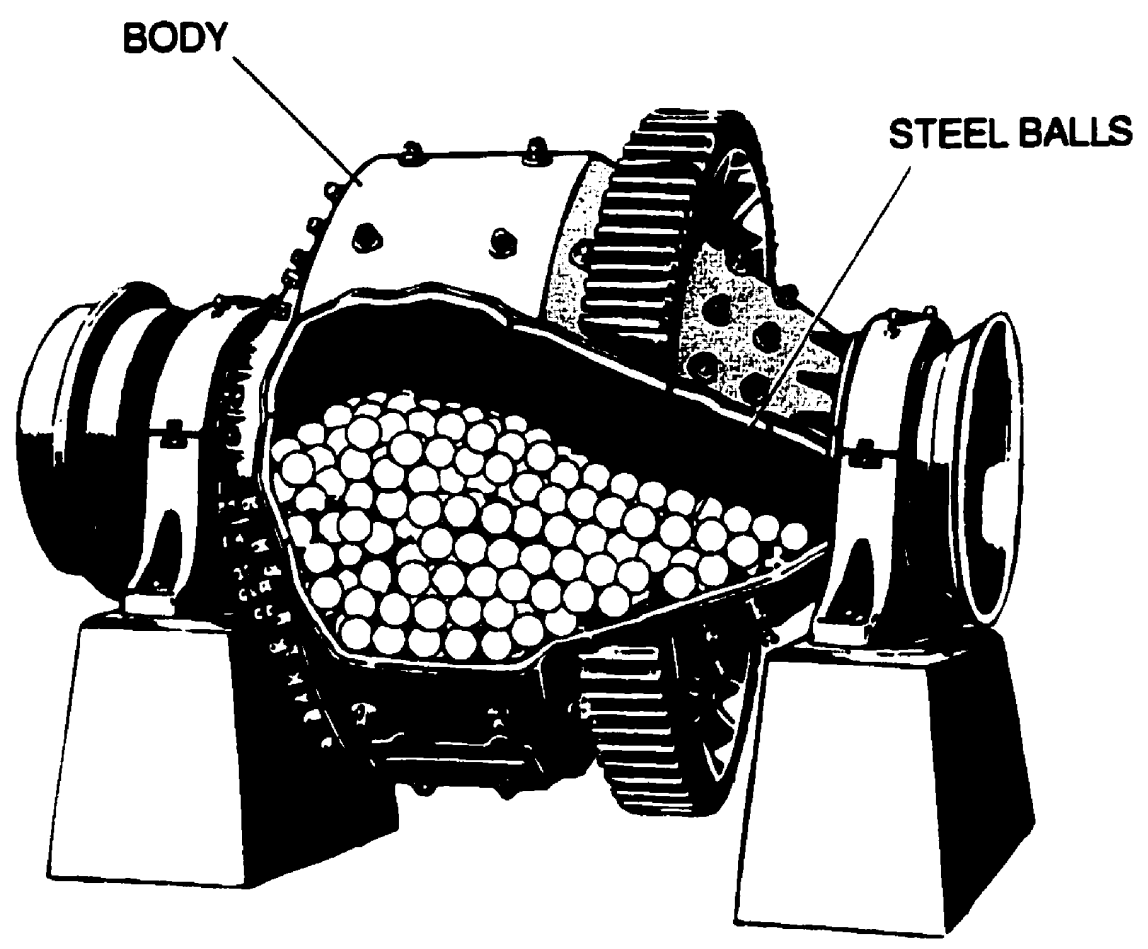

Figure 3 Tumbling ball mill, [ref.6]. 
In vibrating mills, the grinding medium receives rapid impulses at a rate proportional to the vibrating frequency of the mill. Impact forces acting on the powder exceed shearing and friction forces. The entire charge slowly revolves counter clockwise to the oscillatory vibrations, so that grinding and intensive mixing occur simultaneously. Vibratory mills utilize smaller media balls because of higher impact forces, frequencies, and acceleration; thus, a higher specitic surface is available for milling ${ }^{[9.14]}$.

\section{2-6-3 Attrition Mills}

Milling in an attrition mill is affected by the stirring action of an agitator that has a vertical rotating shaft with horizontal arms, Figure 4 . This motion causes a differential movement between the balls and the material being milled, thus providing a substantially higher degree of surface contact than is achieved in tumbler or vibratory mills.

Milling is accomplished by impact and shear forces. The rotating charge of the balls and the milling product form a vortex at the upper end of the stirring shaft, into which the milling product and balls are drawn. Balls travel in various trajectories that collide within the dilated charge of medium and powder. Power input to the attrition mills is used to agitate the medium, not rotate or vibrate the heavy containment vessel. In attrition mills,

constant movement causes impact. Random movement of the balls in different rotational direction produces a shearing action ${ }^{[6.7 .10]}$. 


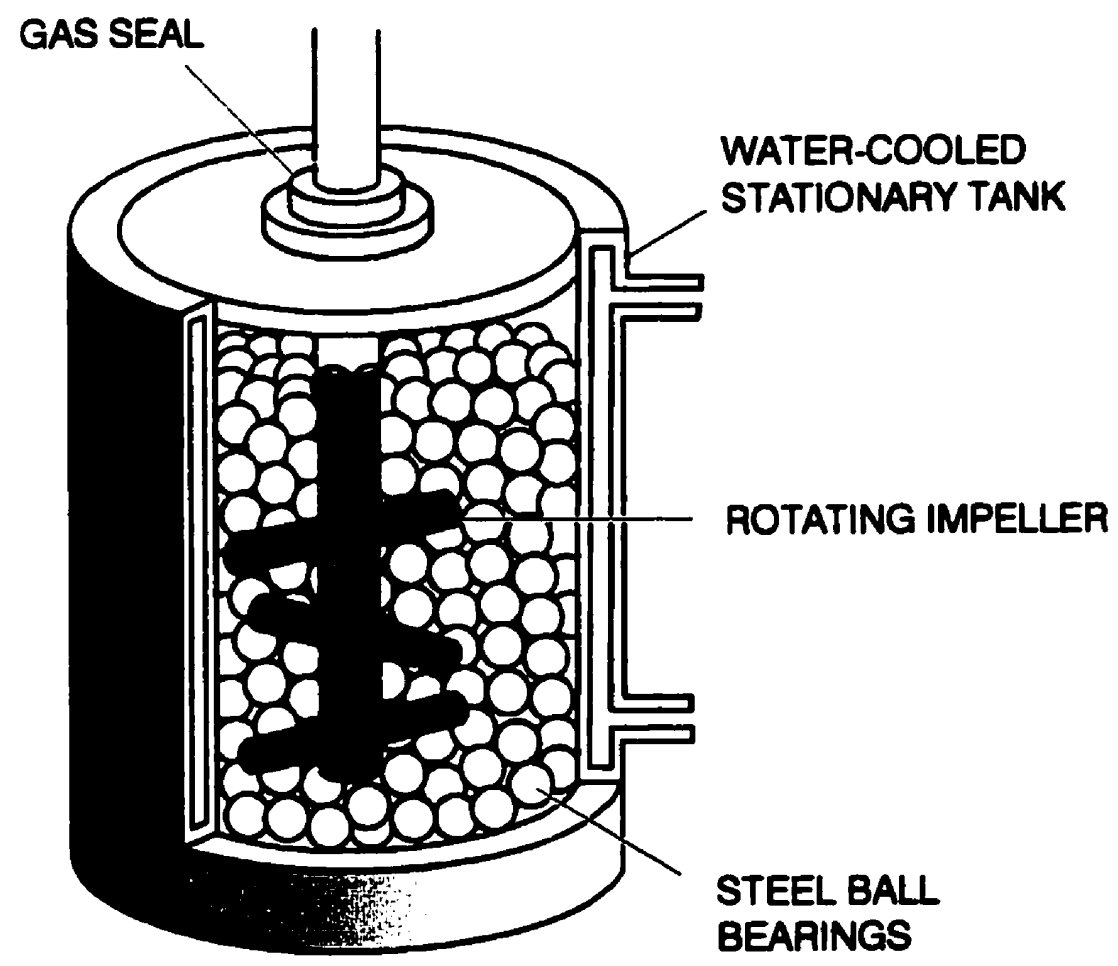

Figure 4 Attrition ball mill, [ref.7] 


\section{2-7 Conventional Ball Mill Specification}

The length to diameter ratio is 1.5 to 1.0 and less. The shell is constructed from a hard ferromagnetic material. The grinding media are made from a hard material and they should be as small as possible for fine grinding. The ratio of the ball size to the ore size can be calculated by the equation $\boldsymbol{d}=\boldsymbol{K} \boldsymbol{D}^{0.5-1}$ where $\boldsymbol{d}$ is the ball diameter, $\boldsymbol{D}$ is the feed size, $\boldsymbol{K}$ is a constant. For fine grinding products the charge volume should be not more than $70 \%$ of the internal volume of the mill. The milling process mechanism is shown in figure 5 . When the weight of the ball is just balanced by the centrifugal force:

$$
\frac{\mathrm{mv}^{2}}{\mathrm{R}}=\mathrm{mg} \cos \alpha
$$

where $m$ is the mass of the ball, $v$ is the linear velocity of the ball; $g$ is the acceleration due to gravity, and $\boldsymbol{R}$ is the radius of the mill, Figure $5, \mathrm{~b}$.

$$
\frac{m v^{2}}{R}=m g \cos \alpha
$$

$\alpha$ is the angle between the line of the centrifugal force and the line of the gravitational force, Figure 5,b.

and

$$
v=\frac{2 \pi R N}{60}
$$

Where $\boldsymbol{N}$ is the number of rotations of the shell in unit time. 


$$
\cos \alpha=\frac{4 \pi^{2} \mathrm{~N}^{2} R}{60^{2} \mathrm{~g}}=0.0011 \mathrm{~N}^{2} \mathrm{R}
$$

When the diameter of the ball is taken into account, the radius of the outermost path is $(D-d) / 2$ where $D$ is the mill diameter and $d$ is the ball diameter and therefore

$$
\cos \alpha=0.001 \mathrm{IN}^{2}\left(\frac{(\mathrm{D}-\mathrm{d})}{2}\right)
$$

The critical speed of the mill occurs when $\alpha=0$, i.e. the ball abandons its circular path at the highest vertical point. At this point $\cos \alpha$ equals 1 .

$$
\mathrm{N}_{\mathrm{c}}=\frac{42.3}{\sqrt{\mathrm{D}-\mathrm{d}}} \mathrm{rev} / \mathrm{min} .
$$

$N_{c}$ is the critical speed of the mill.

The speed at which a ball mill is run is important since it governs the nature of the product. At relatively low speed the medium shapes tends to roll down to the toe of the mill and essentially abrasive comminution occurs. This cascading leads to finer grinding with increased slime production. At higher speeds the medium shapes are projected clear of the crop to describe a series of parabolas before landing on or about the toe of the charge. This cataracting leads to comminution by impact. At the critical speed of the mill, 
the theoretical trajectory of the medium is that which would cause it to fall outside the shell. Centrifuging occurs at higher speeds and the medium is carried around in a fixed position against the shell[ ${ }^{[6,9.11]}$.

During cataracting, the potential energy of the medium is converted into the kinetic energy of impact on the toe. Cascading media utilize energy for abrasive grinding during the decent. Most of the grinding in the mill takes place at the toe of the charge, where not only is there the direct impact of the cataracting medium onto the charge, but also ore packed between the cascading medium shapes receives the transmitted shock ${ }^{[6.9 .11]}$. 


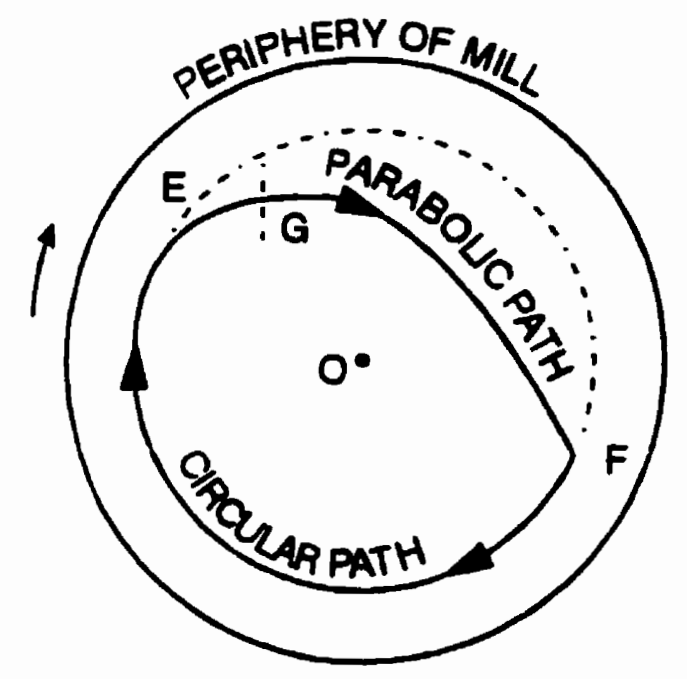

(a) PATH OF TYPICAL BALL IN BALL MILL

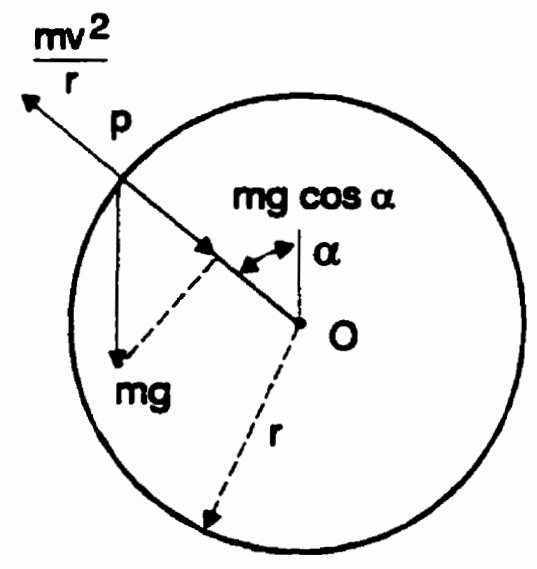

(b) FORCES ACTING ON BALL AT DISTANCE $r$ FROM CENTER OF MILL

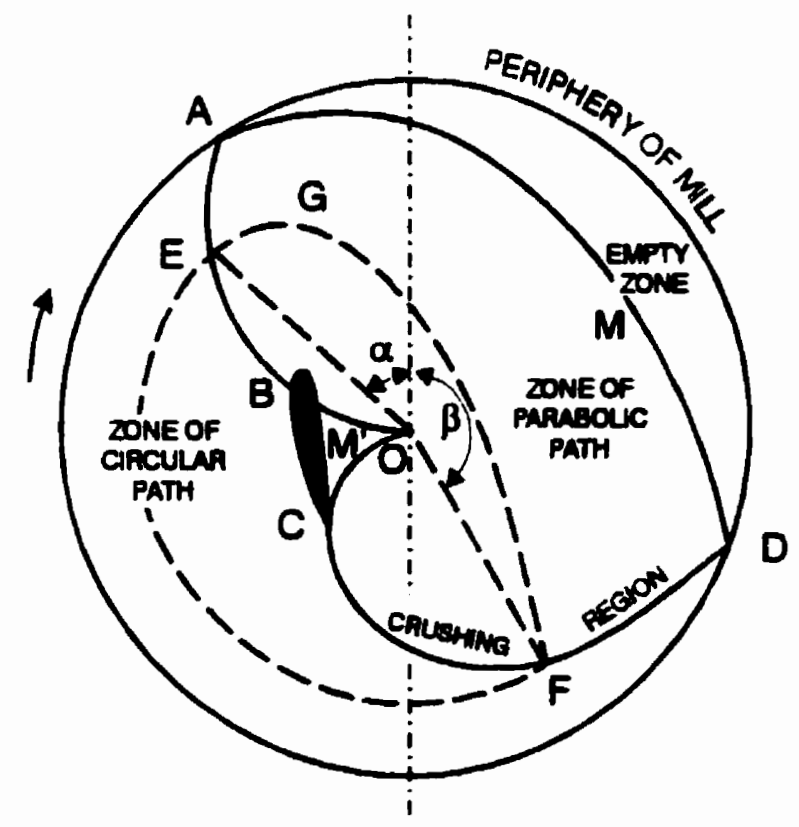

(c) ZONES IN A BALL MILL

Figure 5 Path, forces, and zones of typical ball in a ball mill, [ref.6] 


\section{2-8 Mode of Operation}

In this section, as part of the introduction to the improved ball mill, detailed information will be given on the mode of operation of the conventional ball mill due to its similar mechanism with that of the improved ball mill. The vibrator and the attritor mills have different mechanisms of operation than the improved ball mill; therefore, their mode of operation is not given a detailed explanation in this thesis.

\section{2-8-1 Mode of Operation of the Conventional Ball Mill}

Dry-mills are often employed to produce an extremely fine product. This is because of the high settling speed of the solids suspended in air as compared with the solids suspended in water. The rotation of the mill causes the balls and powders to move in the mill. As the balls tumble down they strike powder that is nipped against other balls. In addition, the general movement of the balls in the bed results in the shearing of particles between balls. Several different types of fracture can occur. Firstly, a massive impact will result in complete disintegration of the particles, (i.e. fracture). Secondly, a glancing blow can chip off a corner of a particle, (i.e. chipping); this mechanism rounds irregular particles into roughly spherical ones. Thirdly, rubbing will cause the wear of surfaces (i.e. abrasion). Milling roughly rounded particles formed by chipping will produce smooth particles similar to beach pebbles. Chipping and abrasion will lead to the production of fine material and their combined effects are referred to as attrition. The bed is in a cascading state at slow speed of rotation. At higher rotational speeds, more of the balls are ejected from the surface at the top of the mill and a cataract of balls is formed ${ }^{[6.15]}$.

Three possible modes of grinding media movement are shown in Figure 6. Under the cascading mode of operation the charge moves counter to the drum rotation, Figure 6,a. 
Velocity gradients in the feed, as well as between the feed and the shell, create favourable conditions for effective attrition. In the cataracting mode of operation Figure $6, b$ the feed is concentrated in the narrow zone, where it tumbles along a curved trajectory. A similar trajectory has been detected in the so-called hurricane mode of operation, Figure 6,c, although in this case the material is distributed over the entire volume of the drum. The hurricane mode of operation is characterised by the combined action of compression. shear and impact stresses. Shear resulting from the attrition of particles caught between balls is the prevailing mode of stress in the attritor mills. The action of shear forces is supplemented with compression ${ }^{[6.11 .16 .17]}$. 


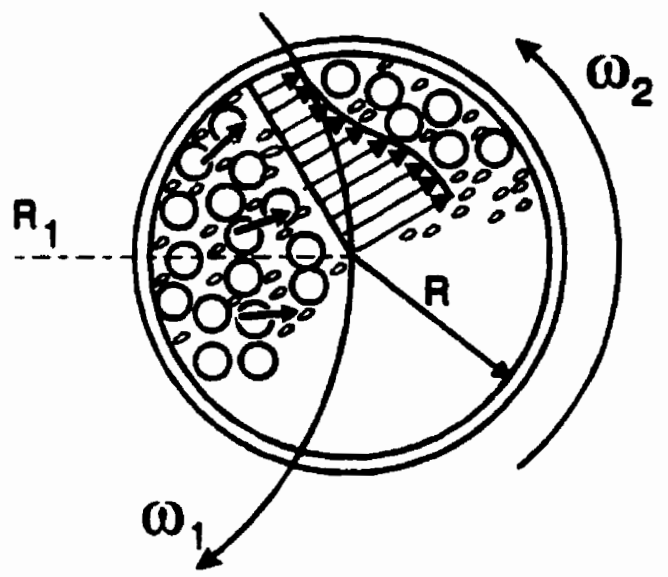

(a) CASCADING

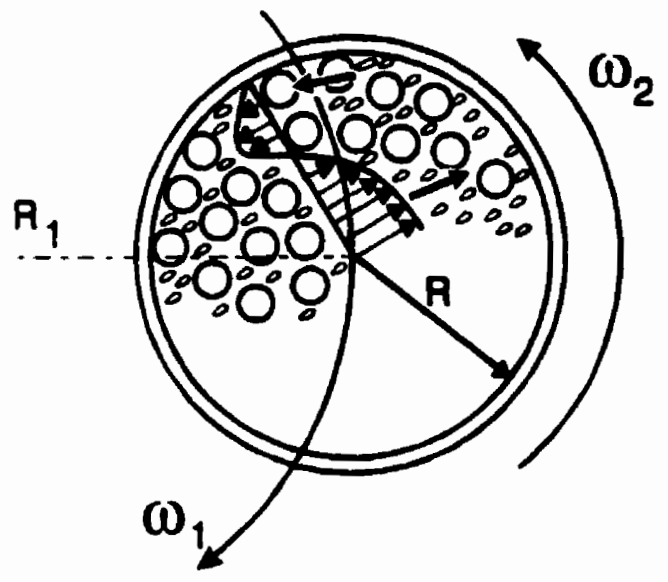

(b) CATARACTING

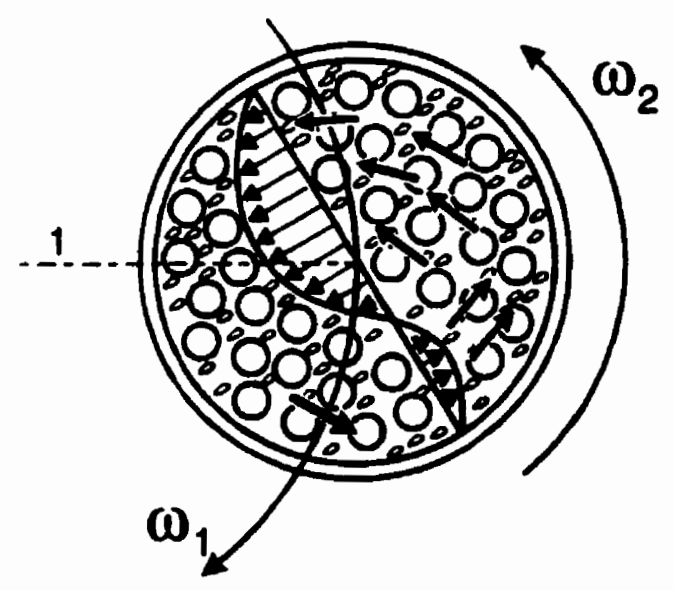

(c) HURRICANE

Figure 6 Types of motion of balls in a planetary mill, [ref.15] 


\section{2-9 Kinetic Energy in Milling Devices}

According to the way the kinetic energy of the drive is transmitted to the grinding media and the material to be ground, the grinding mills can be divided into three main groups:

1- Kinetic energy is transmitted to the mill body, and then it is transferred to the grinding medium and charge to be ground by friction; the charge is subjected to centrifugal and gravitational forces (mills with loose media).

2- Kinetic energy is transmitted directly to the ground particles (various types of peripheral speed and roller mills).

3- Kinetic energy is transmitted directly to the material being ground by means of a flowing stream of the carrier gas which creates vigorous collisions between particles and leads to their impingement on a rigid obstacle (fluid energy mills) ${ }^{[1,8,15]}$.

It has been reported that the grinding effect increases with the density of the grinding medium ${ }^{[6]}$. Selection of the size and the shape of the medium depend upon the properties of the material being ground, and upon the required particle size and/or chemical activity of the final product ${ }^{[1,68,18]}$.

In tumbling ball mills the charge inside the mill chamber performs two relative motions: a rotary motion around the mill axis, and also a planetary motion around the drum axis ${ }^{[9]}$.

In attritors, the material is comminuted by means of free moving beads, which are set in motion by a stirrer. The grinding effect depends upon the stirrer speed, on the stirrer and the chamber geometry. Attritors are used for grinding materials with considerably different properties, e.g. soft pigment, but also very hard ceramics ${ }^{[2,7]}$. 
It is known that the different motions of grinding media in a tumbling mill bring about differences in the mode of grinding action.

1- Under the cascading mode of operation, the feed moves counter to the drum rotation. Velocity gradients in the feed, as well as between the feed and the shell, create favorable conditions for effective attrition.

2- In the cataracting mode of operation, the feed is concentrated in the narrow zone, where it tumbles along a curved trajectory. Impact action and a little shearing dominate the particle size reduction.

3- In the hurricane mode of operation, the material is distributed over the entire volume of the drum. This mode of operation is characterized by the combined action of compression, shear and impact stresses ${ }^{16.8 !}$.

The main problems of grinding techniques and technology have not been solved so far. A challenging task is the design of an energy-intensive grinding mill that could grind large amounts of material continuously. Solutions of this problem are greatly hindered by the intensive stressing of the structural parts and by the extensive wear of grinding tools at high accelerations ${ }^{[15,17,19,20]}$.

\section{2-10 Conversion and Transfer of Energy during Milling}

Theoretically, there are three main stages of conversion and transfer of energy during milling.

1- Conversion of the drive's kinetic energy to a mechanical action.

2- The transfer of the mechanical action to the particles being ground. 
3- Matching the stress produced by the mechanical action to the failure stress of the particles.

Particles are stressed when they come into contact with moving or rigid tools or when they collide with each other. Particles undergo elastic or anelastic deformation with the simultaneous formation of a contact stress field. From the sites of primary concentration of stress energy, waves travel into the bulk, both from the particles and the tools. Depending upon the size, shape and internal structure of the particles and tools, the energy waves are reflected so that the energy distribution is highly structured. While energy waves travel through the particles, energy dissipation takes place, so that a part of the energy is converted into heat ${ }^{[5,6.21]}$.

The elastic stress is completely reversible if the rate of the stressing is low and the force acting does not induce changes in the distribution of magnetic moments or in the concentration and distribution of lattice defects. Variable mechanical stress result after a certain number of cycles in anelastic and/or plastic deformation, depending upon the magnitude of the stress created ${ }^{[16,17\rceil}$.

\section{2-11 Energy Consumed by Material}

As a background to the theory of grinding, basically there have been three proposals of how to determine the energy consumed by a mill. The first was proposed by Rittinger in 1867 , who stated that the area produced by comminution breakage is directly proportional to the useful work input, i.e. "The surface theory" ${ }^{25 !}$.

$$
E=C_{R}\left(\frac{1}{X_{2}}-\frac{1}{X_{1}}\right)
$$


or,

$$
E=C_{R}\left(S_{2}-S_{1}\right)
$$

where $\boldsymbol{E}$ the energy input per unit mass

$\boldsymbol{X}_{l}$ is the initial particle diameter

$\boldsymbol{X}_{\mathbf{2}}$ is the final particle diameter

$\mathbf{S}_{1}$ is the initial specific surtace

$S_{2}$ is the final specific surface

$C_{R}$ is the Rittinger's constant (Energy * Length $*$ Mass $^{-1}$ ).

The second theory proposed by Kick in 1885 stated the work required is proportional to the reduction in volume of the particle concerned, i.e. "The volume theory" $[25]$.

Kick's Law may be set out as

$$
\frac{d E}{d X}=C \frac{1}{X}
$$

or in an integrated form, as

$$
E=-C_{K} \log _{e} \frac{X_{2}}{X_{1}}
$$

or more conveniently, as 


$$
E=C_{K} \log _{10} \frac{X_{1}}{X_{2}}
$$

where $\mathbf{C}_{\mathbf{K}}$ is the Kick's constant with dimensions of Energy * Mass ${ }^{-1}$.The other symbols are as above.

The third theory proposed by Bond 1952, after identifying that the first two theories failed for most grinding calculations, stated that the work input is proportional to the new crack tip length produced and equals the work represented by the product minus the work represented by the feed, i.e. "The third theory of comminution" 26$]$.

$$
W=2 C_{B}\left(\frac{1}{\sqrt{X_{2}}}-\frac{1}{\sqrt{X_{1}}}\right)
$$

From his standard work index calculation, Bond determined the constant values so the formula achieved its well-known form including the work index parameter $\boldsymbol{W}_{\boldsymbol{i}}$.

$$
W=\frac{10 W_{i}}{\sqrt{p}}-\frac{10 W_{i}}{\sqrt{F}}
$$

or more conveniently, as

$$
E=W_{i}\left(\frac{10}{\sqrt{X_{2}}}-\frac{10}{\sqrt{X_{1}}}\right)
$$

where $C_{B}$ is Bond's constant with dimensions of Energy $*$ Length $^{0.5} *$ Mass $^{-1} . \mathbf{W}_{i}$ is Bond's "Work Index" which represents the work required to reduce the size of the charge 
from infinite size to $100 \mu \mathrm{m}$. The dimensions of $W_{i}$ are not the same as those of the $C_{B}$ being Energy / Mass ${ }^{-1}$ and the units are usually quoted in the literature as $\mathrm{kWhr} / \mathrm{st}$.

In Bond's Law the sizes $X_{1}$ and $X_{2}$ are " $80 \%$ passing" sizes i.e. the size in microns of a sieve aperture, which would permit the passage of $80 \%$ of the material.

The work index can be determined in laboratory and was defined by Bond as the quantity of energy in kilowatts necessary to reduce one short ton of material from intinite size to $80 \%$ passing 100 microns $^{[26.27]}$

Holmes Law (1957). Since Bond's Law was published there have been attempts to refine it still further. As, for example, Holmes proposed the form ${ }^{[25 \mid}$.

$$
E=W_{i}\left[1-\left(\frac{1}{R}\right)^{r}\right]\left(\frac{100}{X_{2}}\right)^{r}
$$

where $E$ and $\boldsymbol{W}_{1}$ and $\boldsymbol{X}_{2}$ have the same connotation as in the Bond's Law.

$R$ is the reduction ratio i.e. $X_{1} / X_{2}, r$ is an exponent.

If $r=0$ the equation reduces to Kick's Law.

If $r=0.5$ the equation reduces to Bond's Law.

If $r=1.0$ the equation reduces to Rittinger's Law.

It has been a common practice to regard each of the laws as being the integral of the basic differential equation

$$
\frac{d E}{d X}=-C \frac{1}{X^{N}}
$$

when $N=2$ this is the differential form of Rittinger's Law and when $N=1$ this is the differential form of Kick's Law, and finally when $N=1.5$ this is the differential form of Bond's Law ${ }^{[23]}$. 


\section{III-Design of the Improved Ball Mill}

In designing any type of ball milling a primary task of the process engineer is to size the ball mill for a desired output rate with a desired quality of product. Powder feeds into one end of the mill, passes down the mill receiving breaking actions due to the tumbling balls, and leaves as a product with a finer size distribution. This can be considered as a continuous reactor, where energy input is converted to mechanical breakage action and the reaction accomplished is size reduction. In designing a ball mill the following factors must be considered: ${ }^{[2,4,18]}$

1- Mill size.

2- Mill power, specific grinding energy.

3- Grinding media, size and volume.

4- Recycle classification efficiency.

5- Mill circuit behavior under varying conditions.

6- Mill selection for complex circuits.

7. Economic optimization.

\section{3-1 Concept of the Milling Device}

The device is a planar-type ball mill and consists of a few hardened steel balls confined to a stainless steel, horizontal type chamber. The ball movement during the milling process is confined vertically to the chamber walls, and is controlled by an external magnetic field. The intensity and direction of the field can be externally controlled. By adjusting the spatial dimensions of the magnetic field, the ball trajectories, the impact energy and the shearing energy can be varied. In this design of the mill, 
permanent magnets are used. There are three general patterns of the ball movement that can be achieved using this device.

When the magnet is positioned below the mill, the magnetic field holds the balls in the bottom part of the chamber rotating with a frequency $\omega_{c}$ as shown in Figure 7. Friction causes the balls to rotate in the same direction with the frequency $\omega_{b}=\omega_{c} R / r$, where $R$ is the radius of the chamber. and $\boldsymbol{r}$ is the radius of the ball. Periodically, the outer ball on the right-hand side gets released, completes the most of the circle being pushed against the chamber wall by the centrifugal force, and hits the left-most ball at the bottom. The powder is worked both by impact and by shearing. Two useful variations of this case could be achieved as shown in Figure 7, b\& c. By slowing down the chamber mill rotational speed, a situation may achieved when the ball released from the bottom is not fully pinned to the wall by the centrifugal force and can either hit one of the bottom balls or the opposite chamber wall.

The balls may be confined to the bottom part of the chamber for all time either by increasing the intensity of the magnet or by decreasing the rotational speed, as shown in Figure 7, d. In this case the balls both rotate and oscillate around the equilibrium position at the bottom and the powder is worked mostly by shearing.

In figure 7, e the ball movement caused by the centrifugal force can be halted in two opposite positions, at the lowest, and the highest point inside the chamber. The ball trapped by the magnetic attraction in the upper position rotates with the rotation of the mill chamber and can be released to fall vertically on top of one of the bottom balls. The two colliding balls rotate in the opposite directions, which results in a combination of 

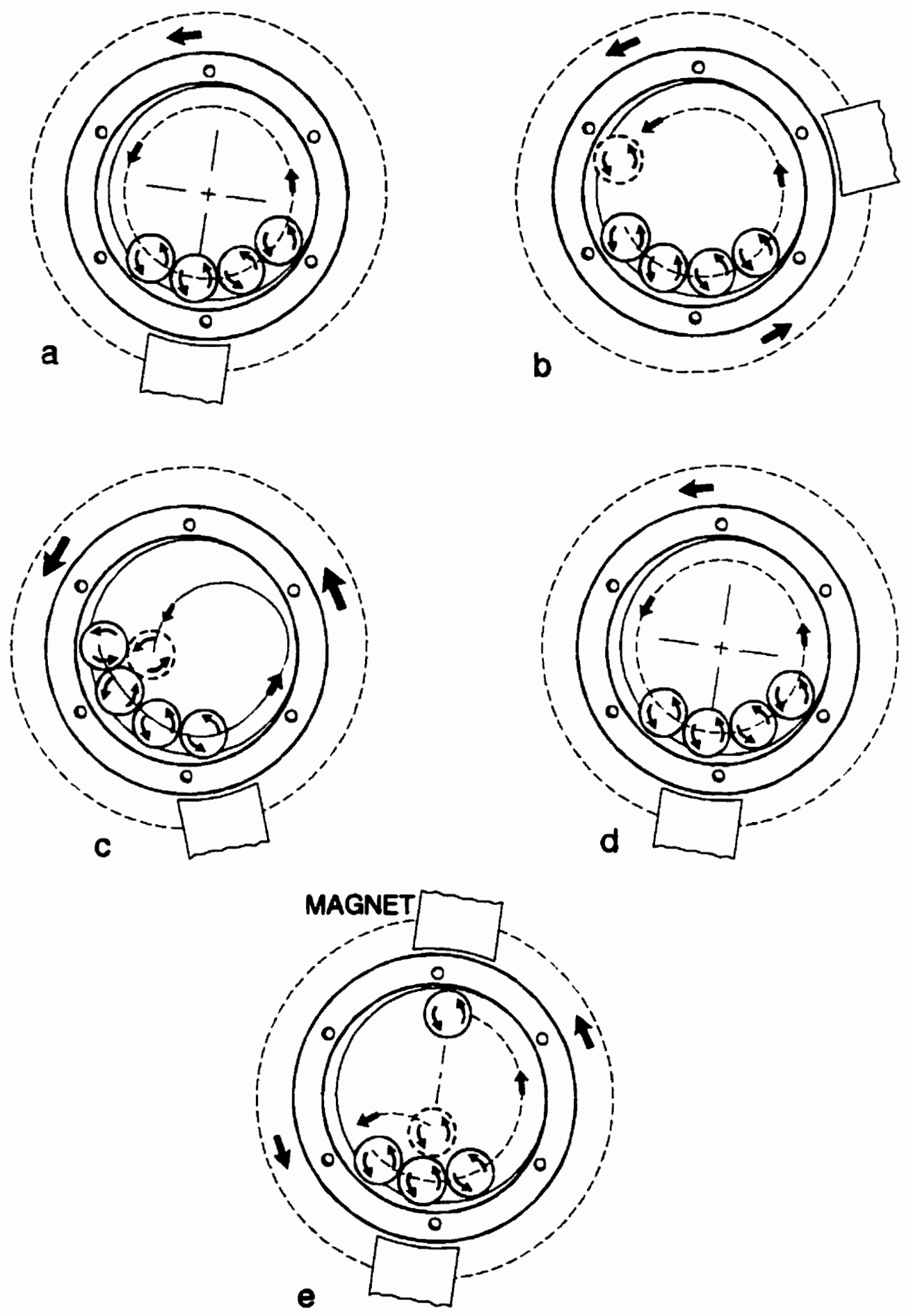

Figure 7 Ball trajectories generated by different intensity and geometry of magnetic field. [ref.3] 
shearing and uniaxial pressure at the surface of contact. The milling conditions in the three cases are similar ${ }^{[3,22]}$.

\section{3-2 Description of the Device}

It is a ball mill comprising a number of hardened steel balls within a cylindrical chamber made of paramagnetic material. The chamber has a substantially horizontal axis of rotation. At least one magnet is mounted outside the chamber to produce a magnetic field within the chamber. The magnet is physically movable, relative to the chamber, between a series of locations on an arc centered on the axis of the rotation of the chamber.

\section{3-3 Design Description}

3-3-1 Shell is in a cylindrical shape made from a hard paramagnetic material, austenitic stainless steel 304.

3-3-2 Size: the design of "Model 2" of the improved ball mill offers different sizes. started from $12.5 \mathrm{~cm}$ in length, $8.75 \mathrm{~cm}$ in diameter as a maximum size and lower through $2.5 \mathrm{~cm}$ adjustment as shown in figure 15 . The test model of the improved ball mill, "Model l" has one size, $3.5 \mathrm{~cm}$ length and $5 \mathrm{~cm}$ diameter.

3-3-3 The magnet holder: In the case of "Model 2" of the improved ball mill, the magnet holder moves up and down by a step of $2.5 \mathrm{~cm}$, and also makes possible the adjustment of the distance between the mill and the magnet. An arc could be built between the upper and the lower holder and have its center of curvature substantially at the axis of rotation of the cylinder. The magnet is physically movable relative to the cylinder over a significant distance between a series of locations. In "Model l" of the improved ball mill, 
the magnet position can be adjusted physically around the mill chamber as shown in Figures $9 \& 10$.

3-3-4 Magnets: The magnets are rectangular, $5 \mathrm{~cm}$ long, $1.25 \mathrm{~cm}$ width, and $1.25 \mathrm{~cm}$ thick.

\section{3-3-5 Marnet Specifications:}

The maximum energy product $(\mathrm{BH})$ max. is 30 MGOe.

The residual induction $(\mathrm{Br})$ is $11000 \mathrm{Gauss}$.

The coercive force is $(\mathrm{Hc})$ is 10000 Oersteds.

The intrinsic coercive force (Hci) is 18000 Oersteds.

\section{3-4 The Improved Ball Mill Test Design}

The test model "Model 1 " of the improved ball mill is shown in Figure 8. It is a cylindrical chamber $5 \mathrm{~cm}$ diameter and $3.5 \mathrm{~cm}$ wide, connected to a motor through a bearing block, pulley, and a belt. The motor speed is controllable. A number of hardened steel balls, $1.25 \mathrm{~cm}$ diameter, are within the mill. Adjustable magnet(s) holder is connected to the base plate, Figure 9. Standoff adjusting screws are fixed on the magnet holder to adjust the distance between the mill and the magnet(s) positions. The face of the mill is made of Plexiglas to monitor the ball movements while adjusting the magnetic field. Pre-set patterns could be determined for each material to be milled or ground. Figure 10 shows an exploded view of this design.

\section{3-5 The Improved Ball Mill Design}

As noted above the mill is a cylindrical shell and must be made from a hard paramagnetic alloy such as austenitic stainless steel. The width of the cylinder of 
"Model 2" of the improved ball is $12.5 \mathrm{~cm}$ and its diameter is $8.75 \mathrm{~cm}$. The cylinder is mounted for rotation about a substantially horizontal axis, which is also the axis of the chamber, Figure 11. Within the shell are number of balls made from a hard ferromagnetic alloy with a diameter of $1.25 \mathrm{~cm}$. The magnet(s) can be repositioned relative to the chamber by movement around the arc which has its center of curvature substantially coincident with the axis of rotation of the chamber, or by physically moving them from one discrete mounting location to another by adjusting the chamber, Figure 11 . The magnet holder can be adjusted to the mill through $2.5 \mathrm{~cm}$ step as shown in Figure 12. The design offers different volumes of the mill as shown in Figures $13,14 \& 15$. By adjusting the lift-side-end of the mill, different volumes of the ball mill are obtained. The adjustment process takes place through $2.5 \mathrm{~cm}$ step. The maximum volume is $12.5 \mathrm{~cm}$ wide by $8.75 \mathrm{~cm}$ diameter, and the minimum volume is $2.5 \mathrm{~cm}$ wide by $8.75 \mathrm{~cm}$ diameter. The criterion used for getting the volume variation is the ratio of length to diameter less than or equal 1.5. The lift-side-end is bored to accommodate a slip fitting, for introducing gas to the mill, Figure 12. The design offers a controllable milling environment from vacuum to some hundred $\mathrm{KPa}$ over pressure of gas (or liquid). Figure 12 shows an exploded view of this design. Front view of the improved ball mill is shown in Figure16, and the plan view is illustrated in Figure 17. 


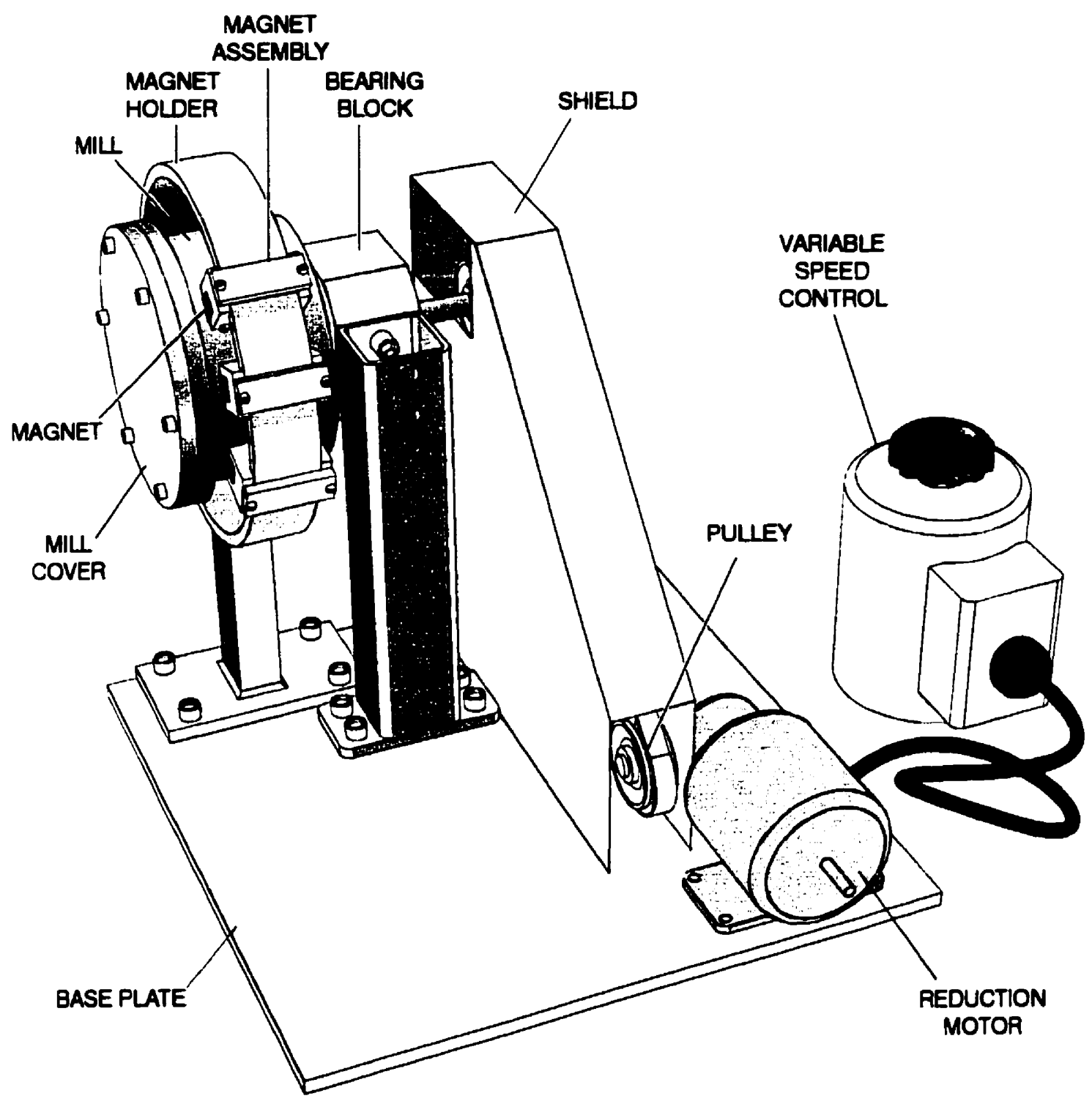

Figure 8 Model 1 of the improved ball mill assembly. 


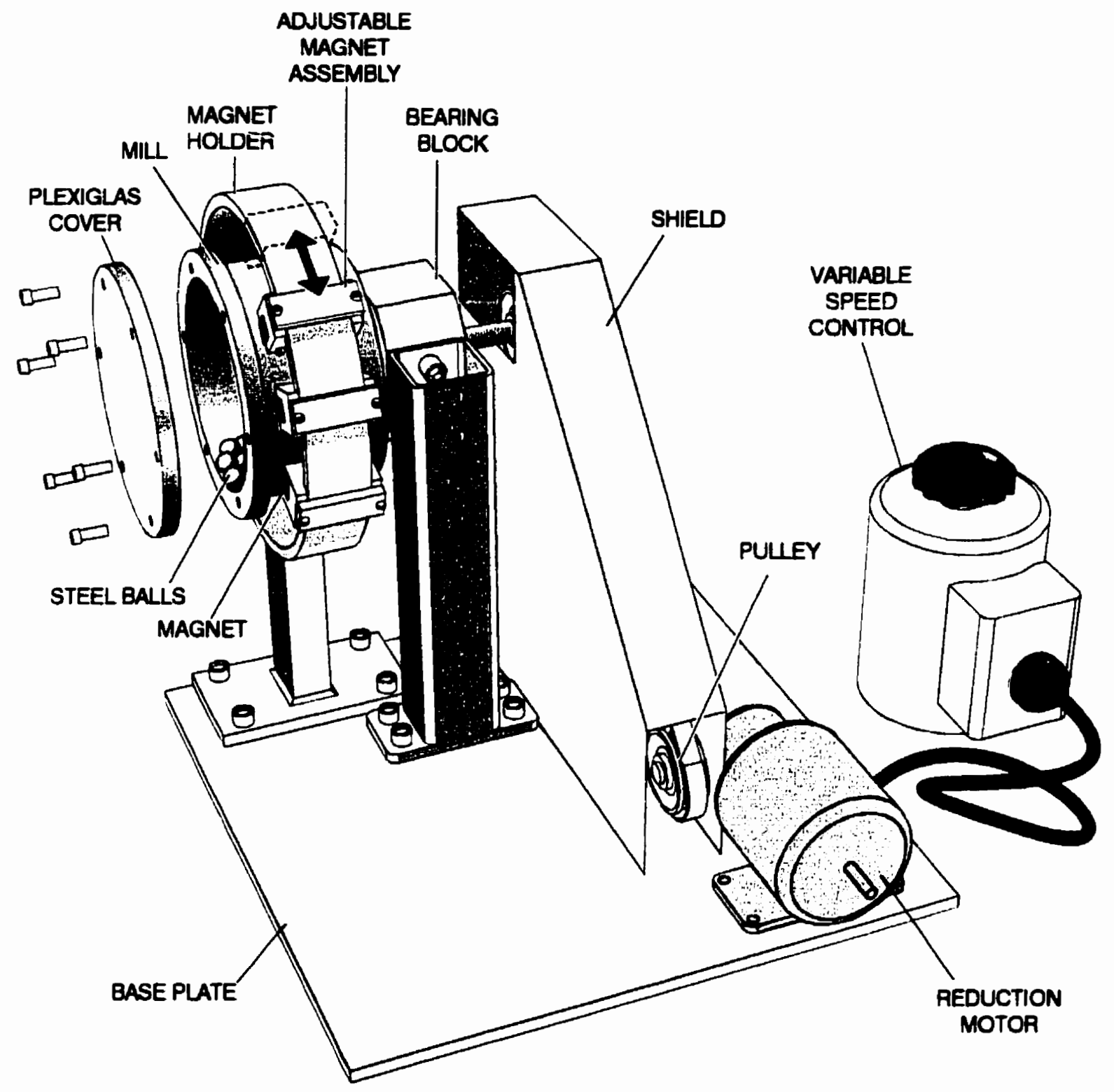

Figure 9 The improved ball mill "Model 1" showing cover removed. 


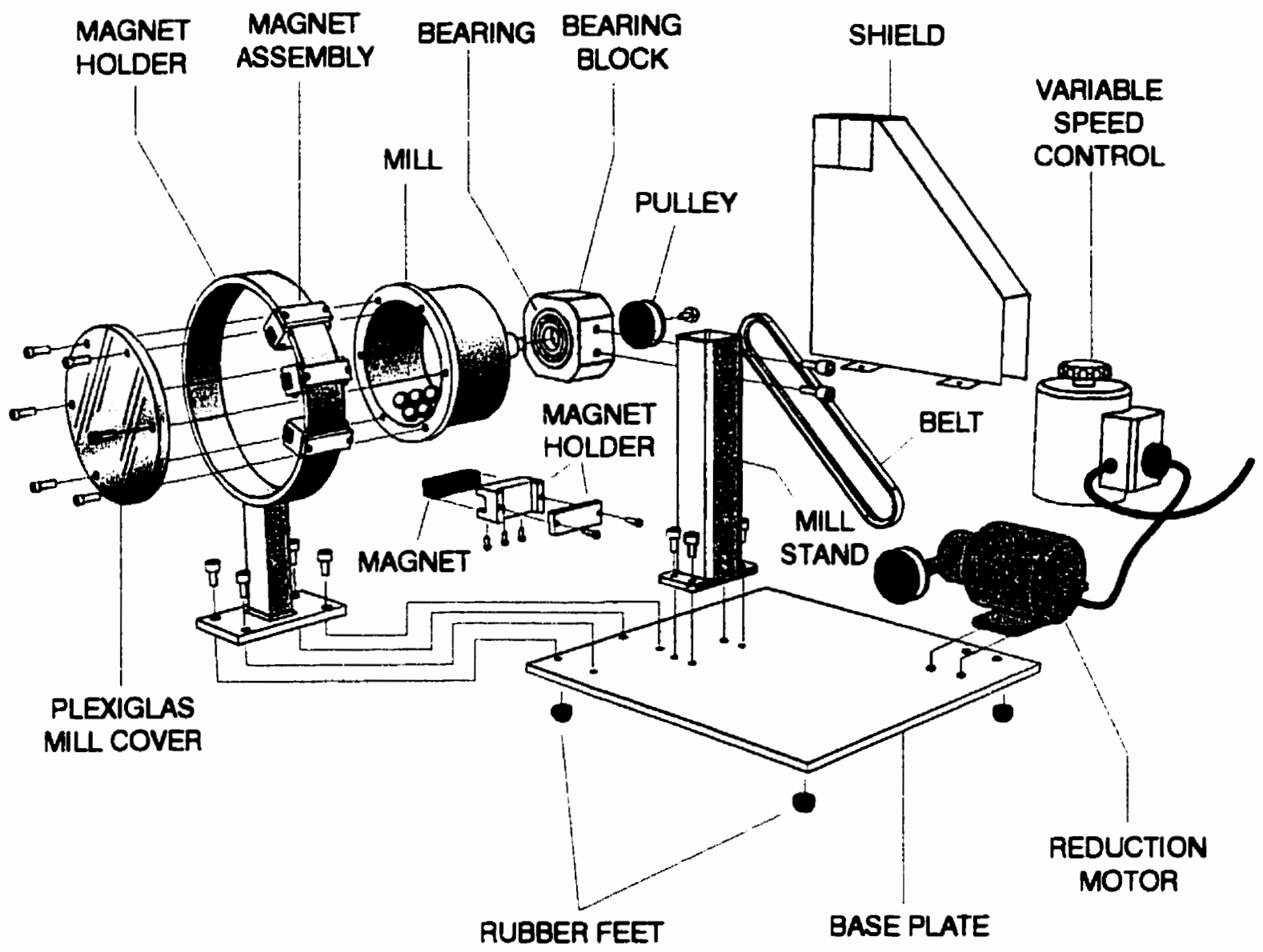

Figure 10 Exploded view of model 1 of the improved ball mill. 


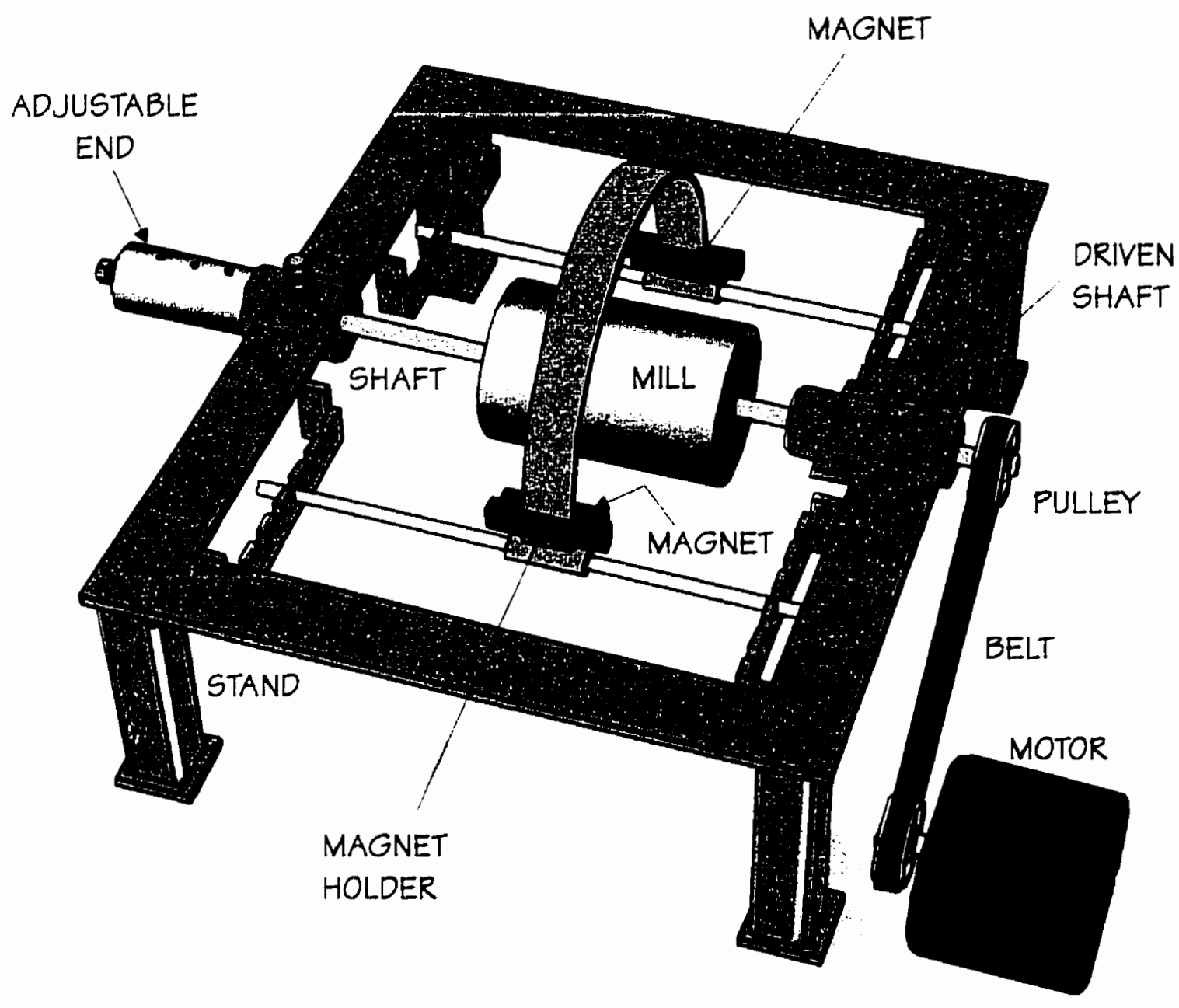

Figure 11 Pictorial view of model 2 of the ball mill. 


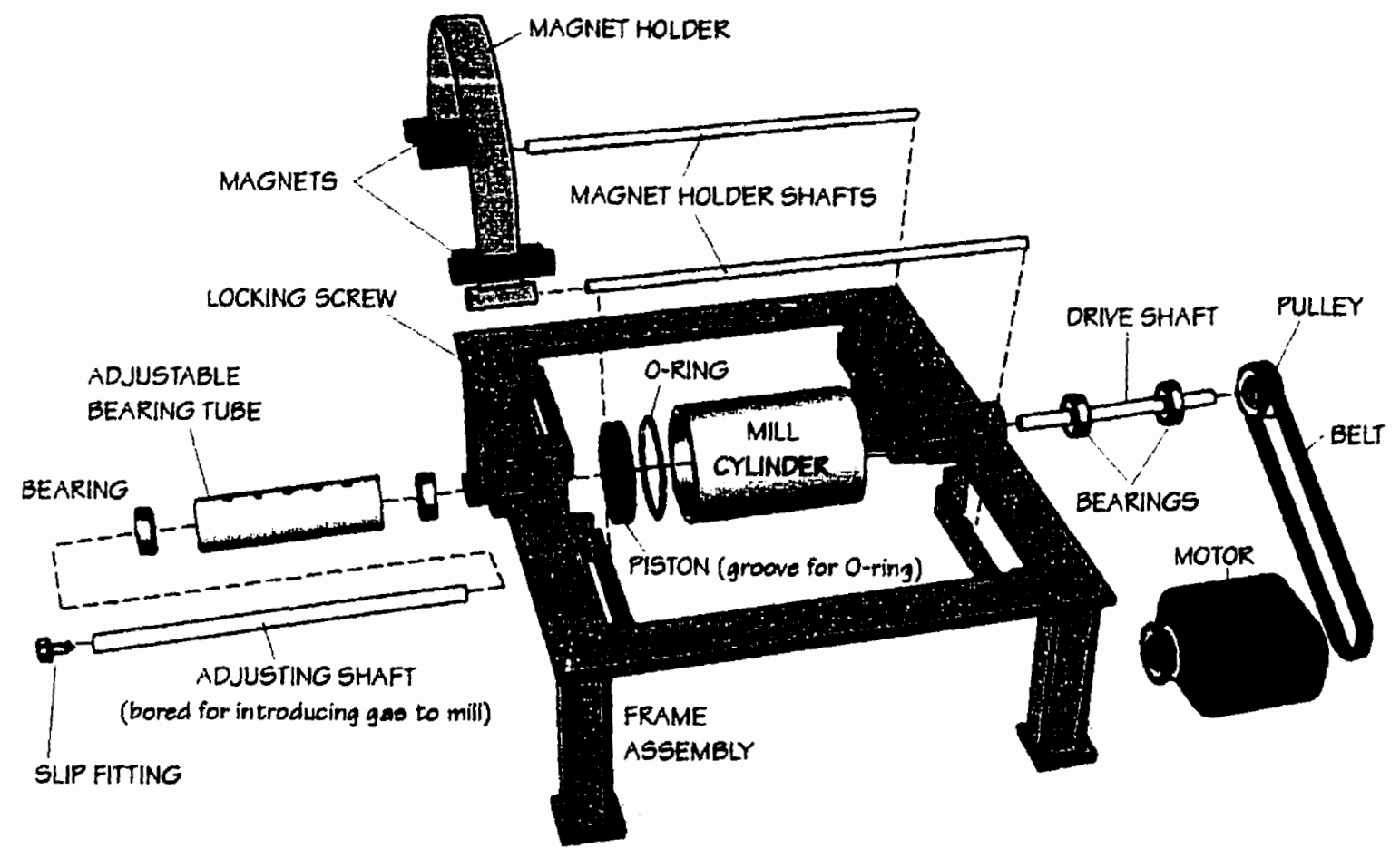

Figure 12 Exploded view of the improved ball mill "Model 2". 


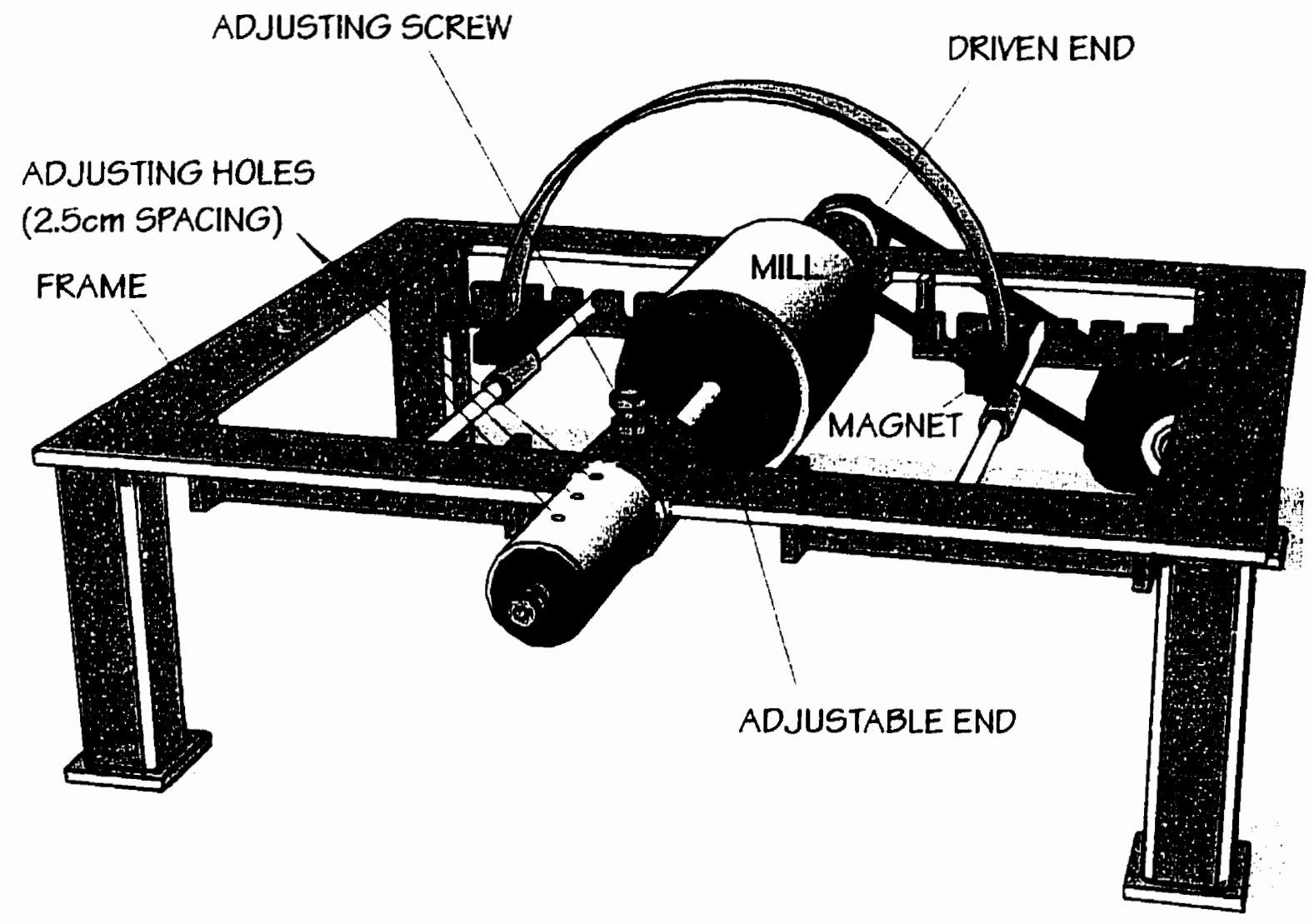

Figure 13 Pictorial view of the improved ball mill "Model 2" showing full $12.5 \mathrm{~cm}$ length. 


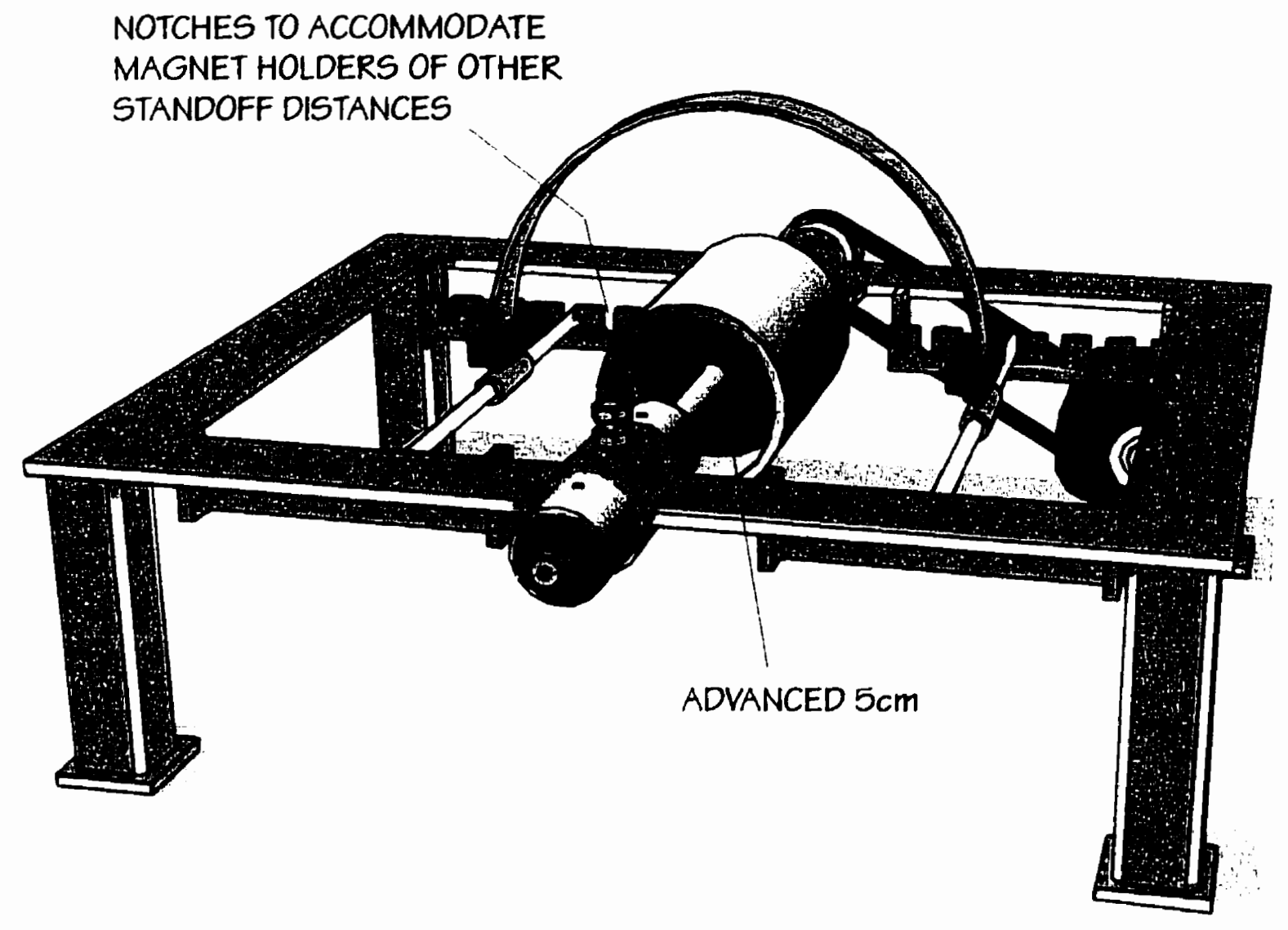

Figure 14 Pictorial view of the improved ball mill "Model 2" showing reduced $5 \mathrm{~cm}$ for $7.5 \mathrm{~cm}$ mill length. 


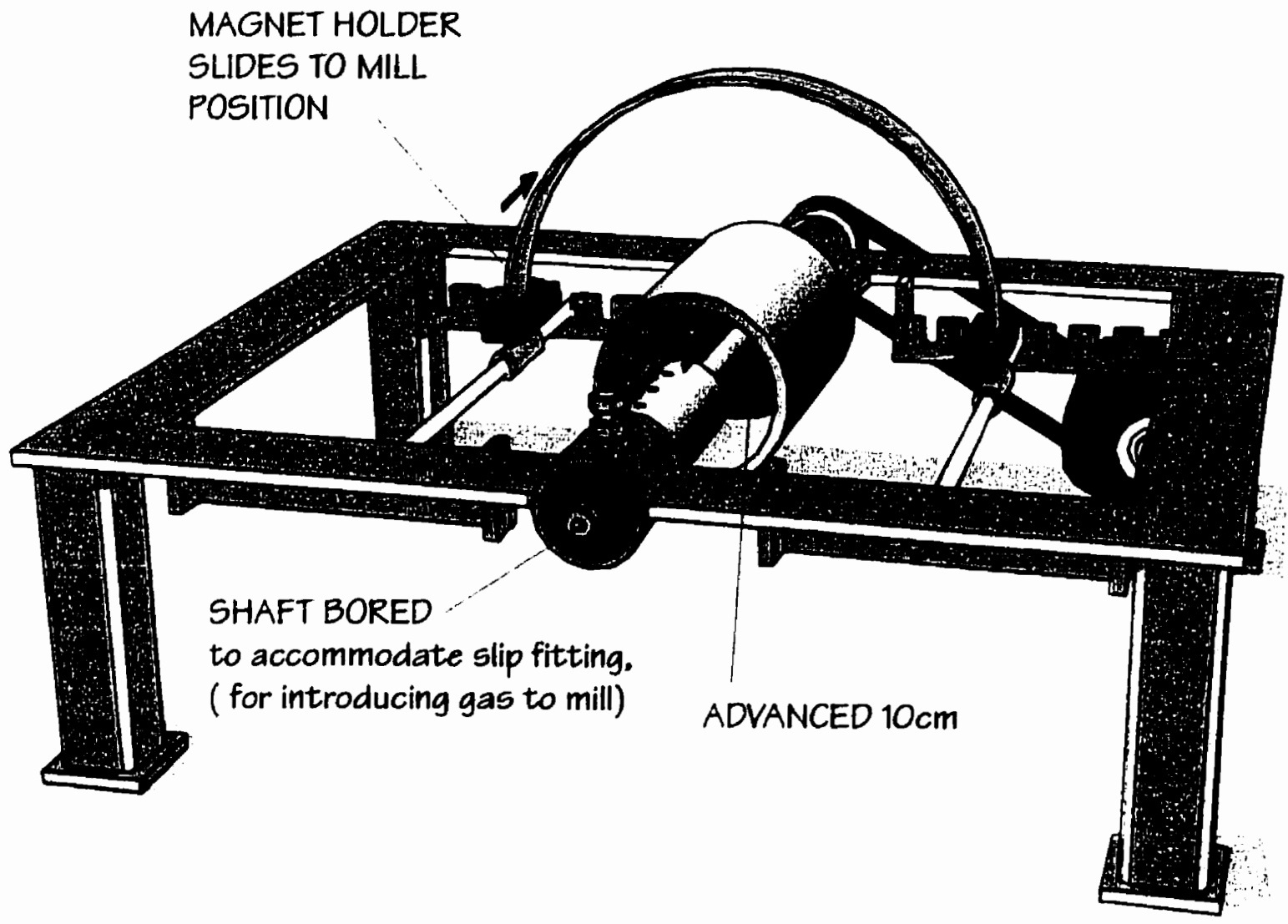

Figure 15 Pictorial view of the improved ball mill "Model 2" showing reduced $10 \mathrm{~cm}$ for $2.5 \mathrm{~cm}$ mill length. 


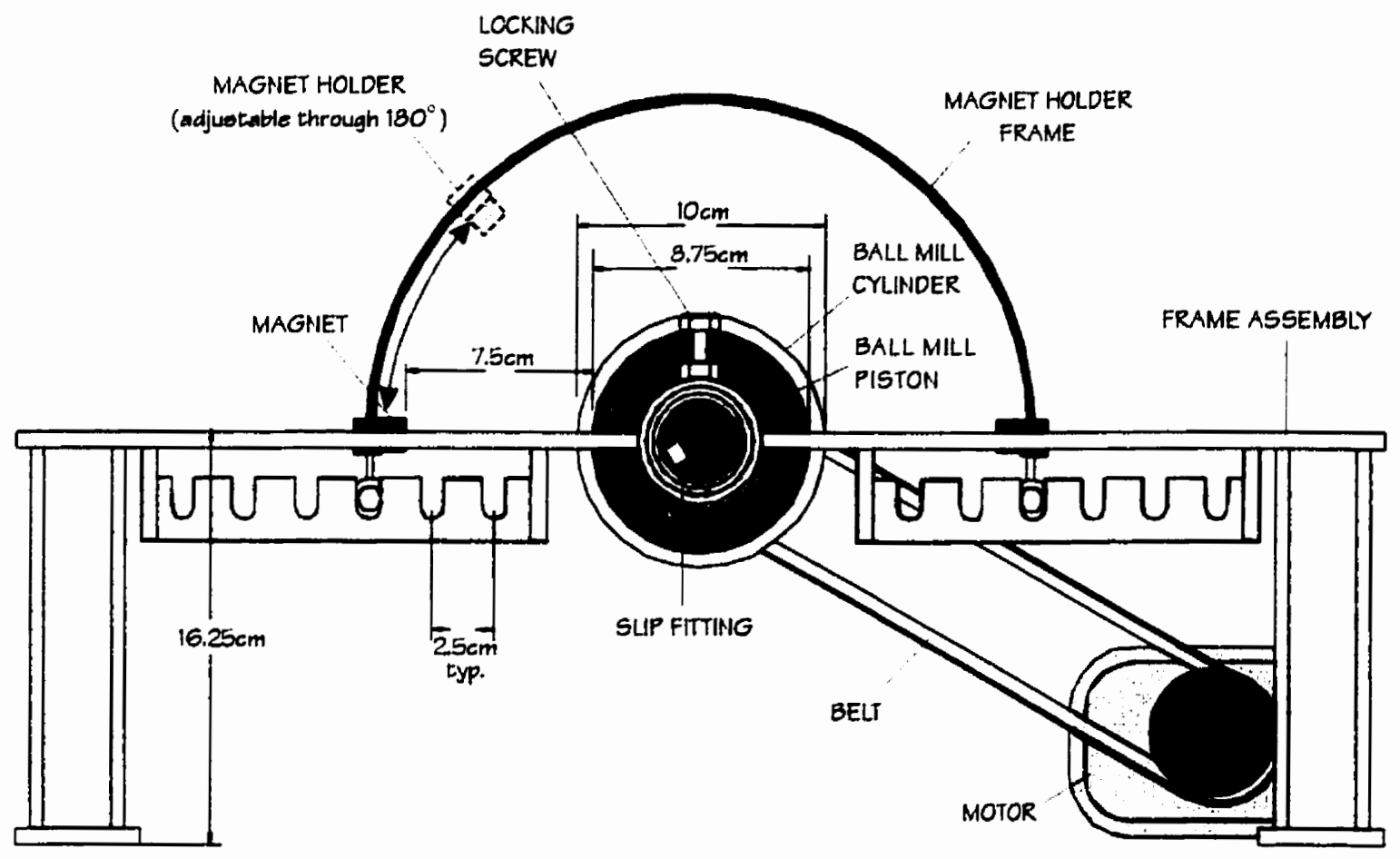

Figure 16 Front view of the improved ball mill "Model 2". 


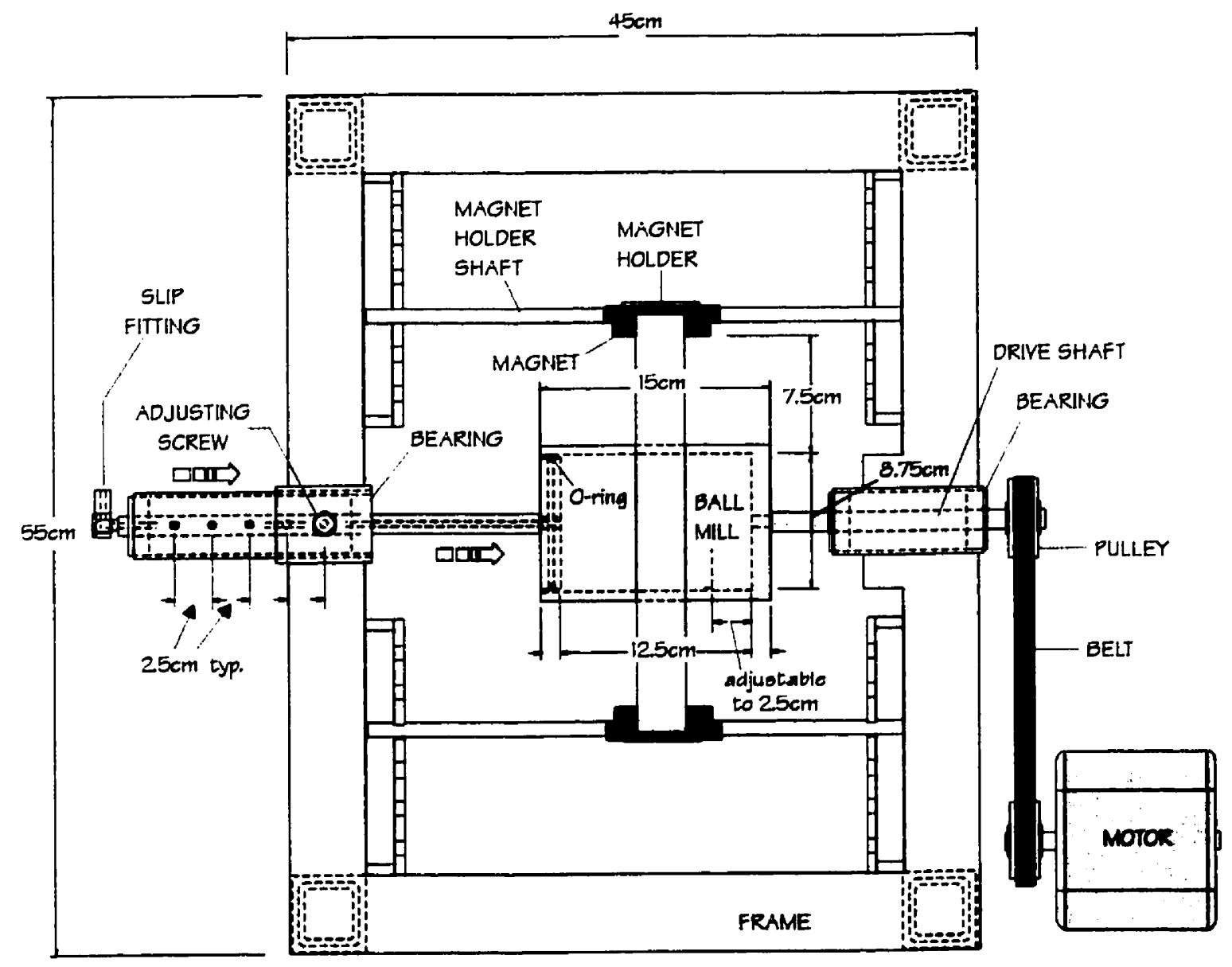

Figure 17 Plan view of the improved ball mill "Model 2". 


\section{3-6 Configuration of the Magnetic Field}

For the production and measurement of magnetic fields and intensity of magnetization, it is desirable to consider quantitatively the fields and forces produced by the magnets.

\section{3-6-1 Field of a Marnet}

Consider a bar magnet $\boldsymbol{N S}$ of pole strength $\boldsymbol{m}$ and interpolar distance $\boldsymbol{l}$, as shown in Figure 18, the magnetic moment $\boldsymbol{M}=\mathbf{I m}$. Using the fundamental relation according to which the field strength varies inversely as the square of the distance $d$ from a pole:

For vacuum

where $\boldsymbol{H}$ is the field strength and $\boldsymbol{m}$ is the pole strength.

When surrounded by a medium of permeability $\mu$.

and adding vectorially the fields that are produced by both poles, it can be shown that the field strength at distance $\boldsymbol{r}$ from the center of the magnet given by:

Provided that $r$ is large compared to $l$, where $r$ is the distance between the center of the magnet and the point $P$, and $\theta$ is the angle between the axis $S N$ of the magnet and the line $O P$ drawn from its center to the point $\boldsymbol{P}$, at which the field is observed. The direction of 
the field at this point is defined by $\varphi$, the angle it makes with $O P$ prolonged, and is determined by $2 \tan \varphi=\tan \theta$

A graphical construction based on this relation may be made easily by trisecting $O P$, so that $O C=C P / 2$, drawing $C D$ at right angles to $O P$ and to cut $S N$ produced at $D$. Then $D P$ is the direction of the field at $\boldsymbol{P}$. There are important special cases, the end-on position (first Gaussian position) for $\boldsymbol{\theta}=\boldsymbol{0}$, and the broadside position (second Gaussian) for $\theta=90^{\circ}$. For these the fields are, respectively,

$$
\begin{aligned}
& H_{1}=\left(\frac{2 M}{r^{3}}\right)\left(\frac{1+l^{2}}{2 r^{2}}\right) \\
& H_{2}=\left(\frac{M}{r^{3}}\right)\left(\frac{1-31^{2}}{4 r^{2}}\right)
\end{aligned}
$$

Where $\mathrm{H}$ is the field strength, $\mathrm{M}$ is the magnet moment and $\mathrm{I}$ is the length of the magnet. When two magnets are placed very closely together end-to-end, with opposite poles $\boldsymbol{m}_{\text {I }}$ and $m_{2}$ and separated by a distance, $d$, which is small compared to the extent of the surfaces, the two magnets are attracted with a force, $f$, that may be very large. For each portion of one of the near by surfaces the corresponding part of the other has an attraction given by the fundamental relation:-

$$
f=\frac{m_{1} m_{2}}{d^{2}}
$$

Summing for the effect of all parts of one surface, $A$, on all parts of the other, one obtains 


$$
f=\frac{A H^{2}}{8 \pi}
$$

This is proportional to the pole area $\boldsymbol{A}$ but is independent of the distance between them as long as this small. Since under these circumstances the lines of flux will go through the surfaces without change, the field strength $\boldsymbol{H}$ in the space will be equal to the induction $\boldsymbol{B}$ in the material at surface, and the force may then be expressed by:

$$
f=\frac{A B^{2}}{8 \pi}
$$

\section{3-6-2 Force on Magnet in Field}

The force on a single magnetic pole of strength $\boldsymbol{m}$ in a field of strength $\boldsymbol{H}$ is $\boldsymbol{f}=\boldsymbol{H m}$, acting parallel to the field vector. In a uniform field, a magnet of moment $\boldsymbol{M}^{\boldsymbol{}}$ is acted on by a torque tending to turn the magnet upon an axis at right angles to its length and to the field so that will be parallel to the field. The magnitude of this torque is $L=M \cdot H \sin \varphi$, where $\varphi$ is the angle between the field and the length of the magnet. If the field is produced at $\boldsymbol{P}$ by a magnet of moment $\boldsymbol{M}$ placed at $\boldsymbol{O}$, as shown in Figure 18, the torque action on $\boldsymbol{M}$

$$
L=M M^{\prime} \sqrt{1+\cos ^{2} \theta}\left(\frac{\sin \alpha}{r^{3}}\right)
$$


If a pivoted magnet is placed in a tield, it will have a natural period of oscillations, $\boldsymbol{T}$, equal to

$$
\mathrm{T}=2 \pi \sqrt{\frac{\mathrm{K}}{\mathrm{MH}}}
$$

Where $\boldsymbol{K}$ is the moment of inertia about its point of suspension.

If an additional known moment of inertia, $k$, is added to that already present, without changing the magnet moment, then this increases the period to $\boldsymbol{T}_{i}$; the expression:

$$
M H=\frac{4 \pi^{2} k}{\left(T_{1}^{2}-T^{2}\right)}
$$

can be used to determine either $\boldsymbol{H}$ or $\boldsymbol{M}$, provided the other is known.

In a non-uniform field a magnet will experience a transitional force as well as a torque, because the force pulling one pole parallel to the field will be opposed by a layer force caused by layer field acting on the other pole. If the gradient of the field is $d H / d x$, the force on a magnet of moment, $M$. is $f=M d H / d x$. When the moment is induced by the field and is therefore $M=I v=H \nu$, the force acting on the volume $v$ is $F=H d H / d x 0^{[23.241}$ 


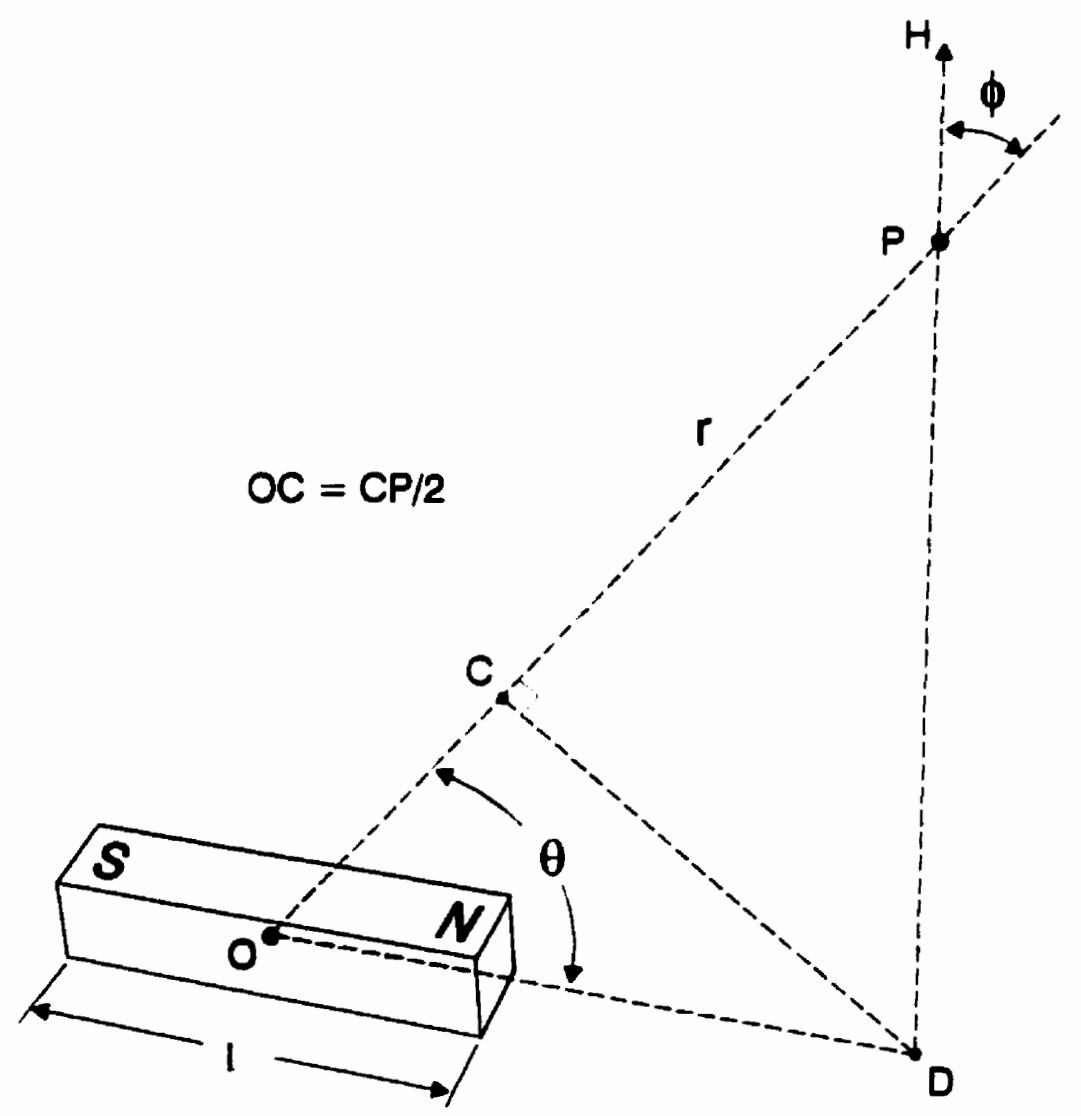

Figure 18 Field produced at any point $P$ by presence of bar magnet NS depends on distance $r$ and angle $\theta$, [ref.23] 


\section{Testing of the Test Model of the Improved Ball Mill}

The main problem of using the existing mills in producing fine materials is the chaotic ball movement, which generates a large range of local milling conditions, and a wide spectrum of particle size distribution. The product of the milling process depends in general on the type of the milling device and the specific milling parameters.

In the improved ball mill, the ball movement during milling is controlled by varying both the strength and /or the positions of the magnets. It involves reproducible and controllable ball movement. In this design, the mill operates at an optimum speed to produce mostly cataracting motion and very little cascading of the balls. The chaotic milling in the existing mills is replaced by highly efficient modes based on carefully controlled ball movement.

Fundamentally, the problem of inefficient chaotic free ball milling has been overcome through the development of this new, improved reactive milling device, which utilises strong magnetic fields. Chaotic milling is replaced by highly efficient modes based on carefully controlled ball movements. Different colours of the steel balls have been used to determine the mode of operation for each case as will be discussed in the next section.

\section{4-1 Mode of Operations:}

4-1-1 Mode A: The ball movement with no magnetic field is shown in Figure 19,case A. After a number of revolutions the trajectories of individuals balls are quite chaotic. With a lower mill speed the balls move along the mill shell to a point where a component of the gravity exceeds the centrifugal force and lets the balls fall from the mill shell. From this point, the balls move in a parabolic path until they hit the mill shell. At higher speed, 
the balls begin to centrifuge and produce mostly cataracting motion and very little cascading action.

4-1-2 Mode B: Introducing a magnet to the ball mill, the ball trajectories are controlled with the magnet field placed at the bottom of the mill as shown in Figure 19, case B. By moving the magnet away / near the mill, different pulling force can be generated. It dramatically affects the ball movement pattern. By adjusting the magnets' positions around the mill, different patterns of operations are generated, as shown in Figure 19 , cases B, C\&D. This results in a characteristic split of rotational movements in opposite directions as illustrates in Figure 19, case B.

4-1-3 Mode C: By slowing down the mill rotational speed, or by reducing the value of the magnetic field, the balls both rotate and oscillate around the equilibrium position at the bottom. In this case the powder particles trapped between rolling balls are mostly affected by shearing, Figure 19 , case $\mathrm{C}$.

4-1-4 Mode D: Further reduction of the pulling force creates characteristic ball movement seen in Figure 19, case D. The individual balls rotate in one direction, while the top ball falls down to the bottom over the others. This cluster like structure creates sliding movement that rotates and oscillates up and down generating additional shearing between the ball and the inner mill wall.

4-1-5 Mode E: Figure 19, case E shows three magnets configured with closed geometry magnetic field. It causes the ball trajectories to be more uniform and make the balls fall under a smaller angle. The "falling down" balls hit balls rotating in the opposite direction at the bottom, consequently strong impact collisions and shearing action is created between the colliding balls. 

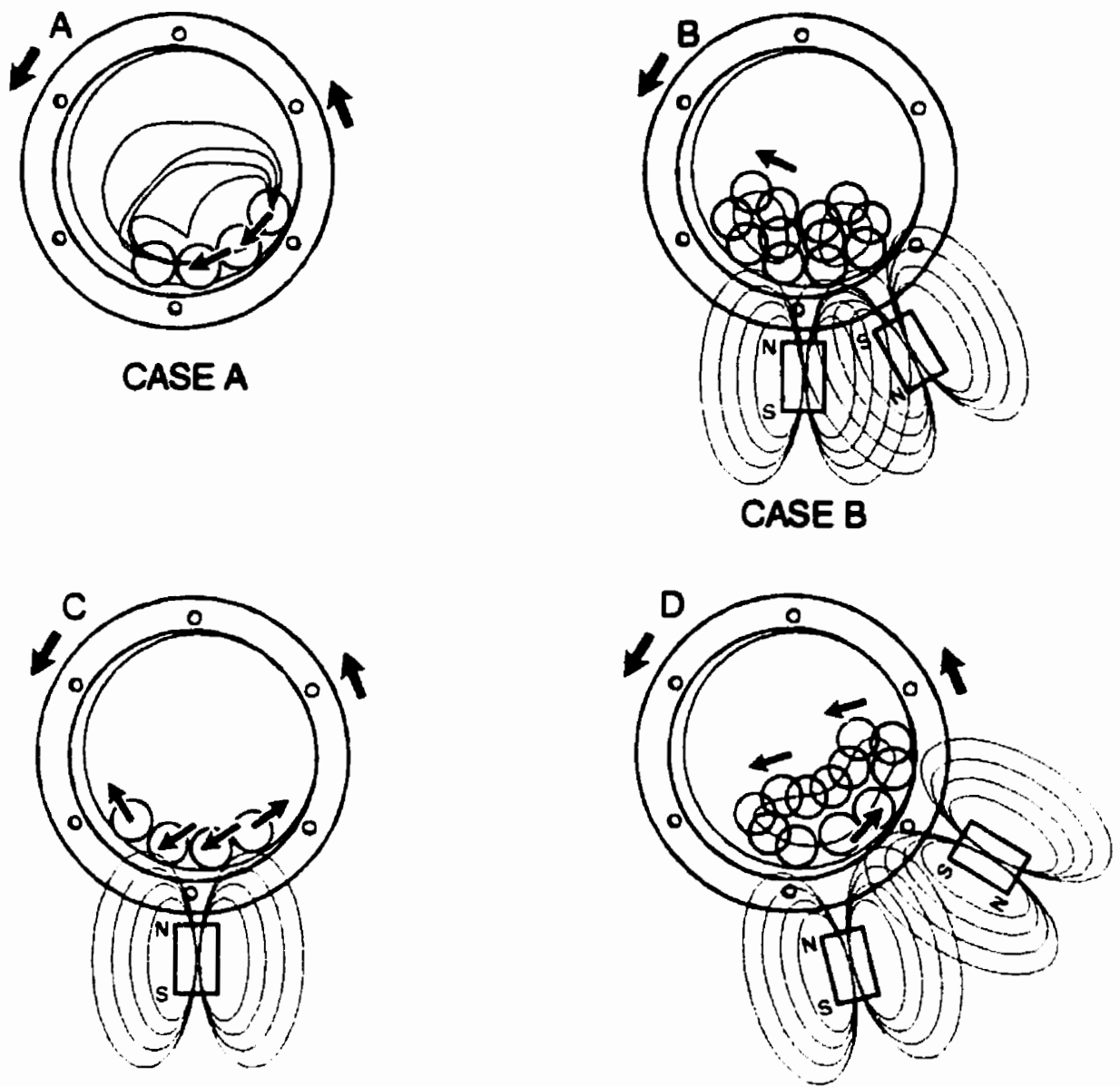

CASE C

CASE D

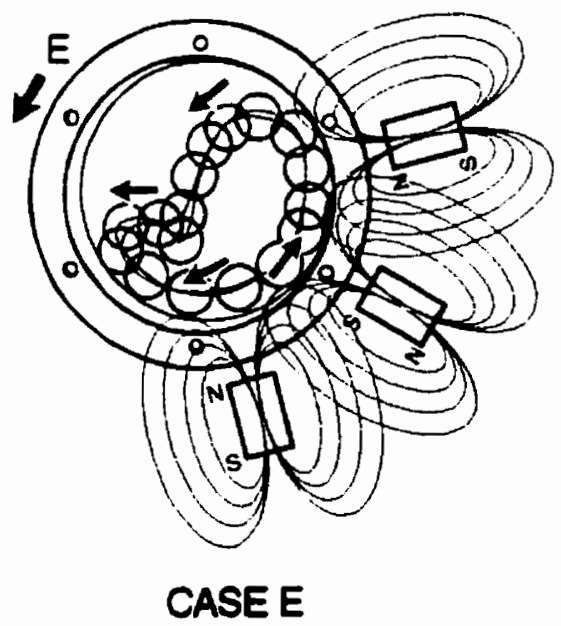

Figure 19 Modes of operation of model 1 of the improved ball mill. 
The unique feature of this design is the specific ball movement pattern in every mode of operation is well defined and highly reproducible. This contrasts with the chaotic and unpredictable ball movement characteristic of the other ball-milling devices.

\section{4-2 Experimental Procedures and Results}

Ball milling experiments were performed using a built in -Nicol- Hall milling device. The device has been used in two different modes. It may be used as a conventional ball mill, case A, Figure 19, or as an improved ball mill, case E, Figure 19. A comparison of the two modes of operation may be conducted by noting the energy consumption of the milling operation, the particle size reduction, the effect of rotational speed, the weight of media, the weight of sample, and the effect of the magnetic force on the grinding ability.

Grinding in a ball mill is affected by point contact of balls and charge particles and given time, any degree of fineness can be achieved. The process is completely random in a conventional ball milling and controllable in an improved ball milling. The probability of a fine particle being struck by a ball is the same as that of a coarse particle. The grinding occurs by the repeated welding and fracturing of the ground material in the ball mill. Each time a powder particle is trapped between grinding balls, it is fractured and is plastically deformed to reduce its size.

\section{4-2-1 Critical Speed}

The work input to a mill increases in proportion to the speed, so the ball mills are run at as high a speed as is possible without centrifuging. Normally this is $65-80 \%$ of the critical speed, the higher speeds often being used to increase the amount of cataracting 
taking place in order to break coarse particles. The critical speed of the tested ball mill "Model 1 " in terms of revolutions per minute is calculated using the formula ${ }^{[6]}$ :

$$
\mathrm{N}_{\mathrm{c}}=\frac{300}{\sqrt{\mathrm{D}-\mathrm{d}}}
$$

Where $N_{c}$ is the critical speed, rpm, $D$ is the internal radius of the mill; $d$ is the radius of the medium. The radii are expressed in centimeters; the critical speed for this mill equals $126.5 \mathrm{rpm}$. Three values of rotational speed are used; the $70 \%$, the $80 \%$, and the $87 \%$ of the critical speed at different media loading, $200 \mathrm{~g}, 350 \mathrm{~g}$, and $750 \mathrm{~g}$.Results of all the runs are shown in Appendix A-D.

\section{4-2-2 Effect of Rotational Speed at 350 arams Media Loading}

4-2-2-1 Conventional Ball Mill: The weight of the sample was 50 grams and the weight of media was 350 grams, the grinding time range is 5 to 30 minutes as illustrated in Table 1. All other parameters are kept constant between comparative tests.

Tablel Rotational speed effect on the particle size reduction in a conventional ball mill.

\begin{tabular}{|l|l|l|l|l|}
\hline Grinding time & Feed & $70 \%$ of the C.S. & $80 \%$ of the C.S & $87 \%$ of the C.S \\
\hline Minutes & $80 \%$ Passing & $80 \%$ Passing & $80 \%$ Passing & $80 \%$ Passing \\
\hline 5 & 1200 & 1140 & 1100 & 1050 \\
\hline 10 & 1200 & 1090 & 1020 & 980 \\
\hline 15 & 1200 & 1000 & 970 & 880 \\
\hline 20 & 1200 & 930 & 840 & 790 \\
\hline 25 & 1200 & 810 & 720 & 670 \\
\hline 30 & 1200 & 760 & 680 & 610 \\
\hline
\end{tabular}

* C.S. is the standard for the critical speed in this table. 


\section{Effect of Rotational Speed}

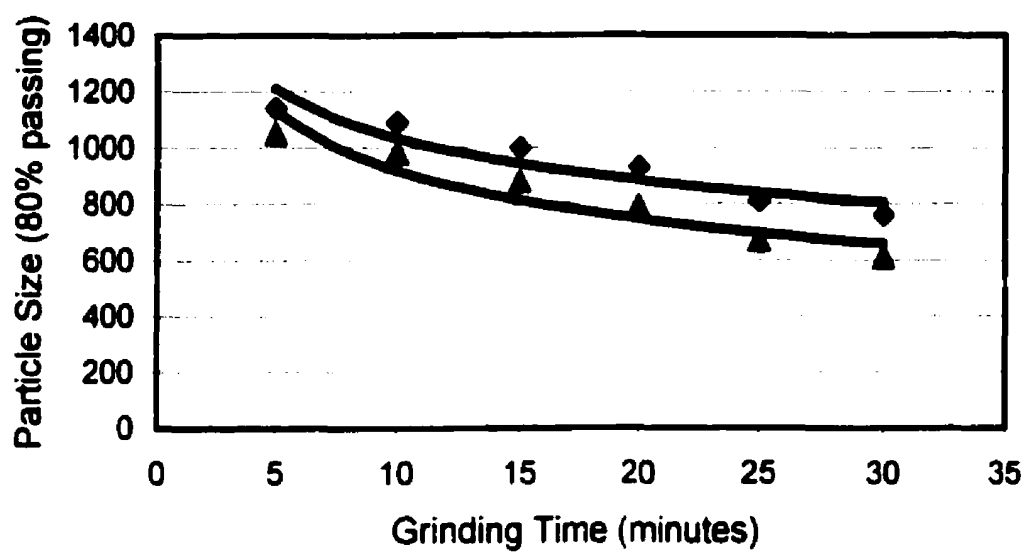

$70 \%$ C.S

$\Delta 87 \%$ C.S.

Figure 20 shows the effect of the rotational speed on the conventional ball mill ability in the grinding process.

4-2-2-2 Advanced Ball Mill: The sample weight is 50 grams, the weight of media 350 grams, the range of grinding time varies from 5 to 30 minutes. All the other parameters kept constant.

Table 2 Efiect of rotational speed on the particle size reduction in the improved ball mill.

\begin{tabular}{|l|l|l|l|l|}
\hline Grinding Time & Feed $(\mu \mathrm{m})$ & $70 \%$ of the C.S. & $80 \%$ of the C.S. & $87 \%$ of the C.S. \\
\hline Minutes & $80 \%$ passing & $80 \%$ Passing $(\mu \mathrm{m})$ & $80 \%$ Passing $(\mu \mathrm{m})$ & $80 \%$ Passing $(\mu \mathrm{m})$ \\
\hline 5 & 1200 & 1120 & 980 & 930 \\
\hline 10 & 1200 & 980 & 810 & 720 \\
\hline 15 & 1200 & 860 & 640 & 560 \\
\hline 20 & 1200 & 800 & 510 & 470 \\
\hline 25 & 1200 & 620 & 400 & 380 \\
\hline 30 & 1200 & 470 & 360 & 310 \\
\hline
\end{tabular}

- C.S. is the standard for the critical speed in this table. 


\section{Effect of Rotational Speed}

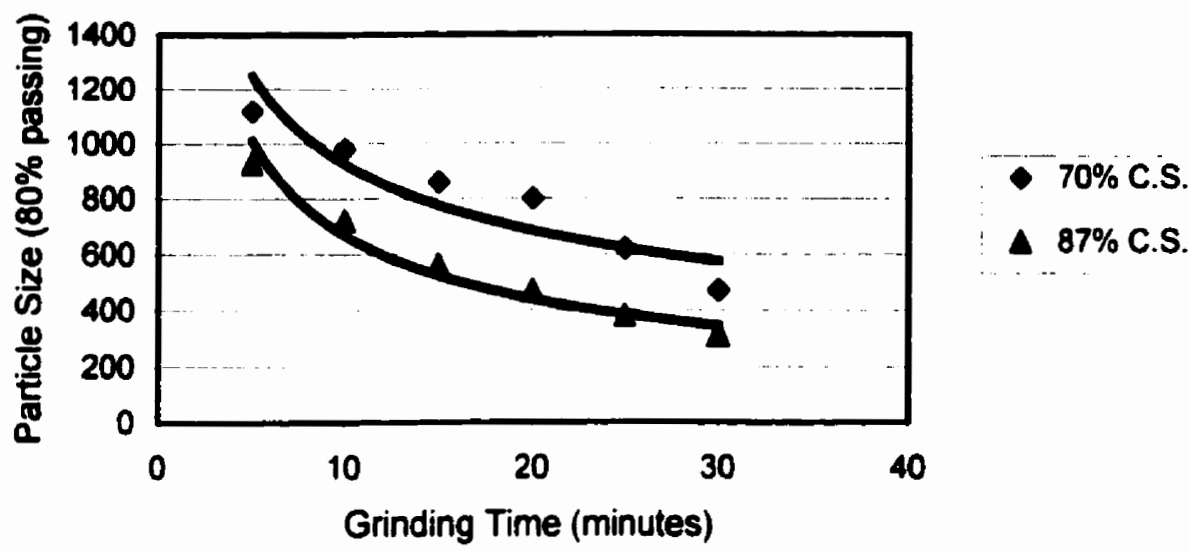

Figure 21 Effect of rotational speed on the ability of grinding in the improved mill.

Effect of Rotational Speed

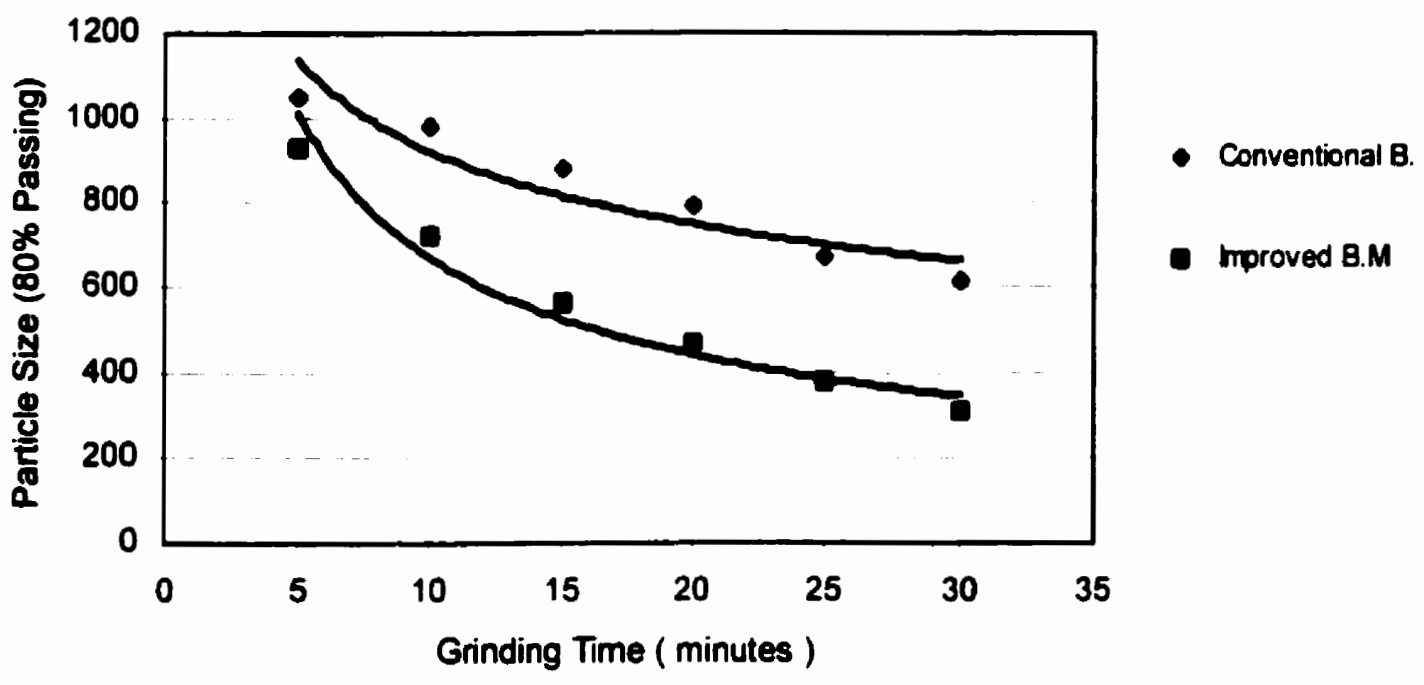

Figure 22 Comparison between the improved and conventional modes of operation at $87 \%$ of the critical speed in terms of the grinding ability under the same conditions of operation.

From the above figures the introduction of the magnetic force improves the ability of the improved ball mill at the different values of the running speed of the mill. From Figure 
22 of the comparison at $87 \%$ of the critical speed, the ability of the improved ball mill is 2.45 times the ability of the conventional ball mill in producing finer and more homogeneous product.

\section{4-2-3 Particle Size Reduction}

When a ductile material is stressed to failure it separates into two pieces. When brittle material is stressed to failure, many product pieces are generated and these pieces have different sizes. The paths of fracture within the material cannot be controlled. The problems of grinding materials are the problems of causing fracture within limits without control of the fracture process itself. Size reduction of any material usually involves cutting, chipping and grinding mechanisms, and the principles of these methods could be considered part of the spectrum of the size reduction methods ${ }^{|6|}$. In the improved ball mill, varying either the strength and/ or the direction of the magnetic field controls the ball movement. This enables selection of both milling energy and degree of shearing and/or impact, and as a result, control of the size reduction limit is achieved. Table 3 shows a comparison between the improved ball mill and the conventional ball mill in terms of particle size reduction under the same conditions of operation. From Table 3, it is clear that the ability of the improved ball mill is better on comparison with the ability of the conventional ball mill in terms of size reduction. Most of grinding in the improved ball mill has been done at the expense of the coarser particle sizes because of the cataracting action. This result in a narrow range of particle size. Grinding with the mill operating as a conventional ball mill has been done at the expense of the smaller particle sizes because of the abrasive action due to the balls cascading. This results in a wide 
range of particle size. The improved ball mill shows its ability of producing a narrow range of particle size.

\section{4-2-3-1 Expressions of Size Distribution}

There are four ways of expressing the cumulative weight distribution associated with a given particle size ${ }^{[25]}$ :

1- The ratio of the sum of the weights of particles smaller than $\mathrm{x}$ in the sample to the total weight of the sample.

2- The ratio of the sum of the weights of particles larger than $\mathbf{x}$ in the sample to the total weight of the sample.

3- Weight percentage of particles smaller than $\mathrm{x}$.

4- Weight percentage of particles larger than $\mathrm{x}$.

In general the particle size distribution expressed by the formula:

$$
Y=f\left(\frac{x}{\bar{x}}\right)
$$

Where $\boldsymbol{Y}$ is fraction of the weight of a sample associated with a particle size $\boldsymbol{x}$.

$\boldsymbol{x}$ is the given particle size, $\bar{x}$ is the reference particle size

The sample is so large in relation to the size of any particie in that the size distribution represents a continuum and thus the weight of particles of size $x$ is $d x^{[25] .}$

The comparison of the particle size reduction is shown on Figure 23 at $87 \%$ of the critical speed, 50 grams of sample weight and 350 grams media .As illustrated on the figure the difference is significant. Results of all the runs are shown in Appendix A-E. 
Particle Size Reduction

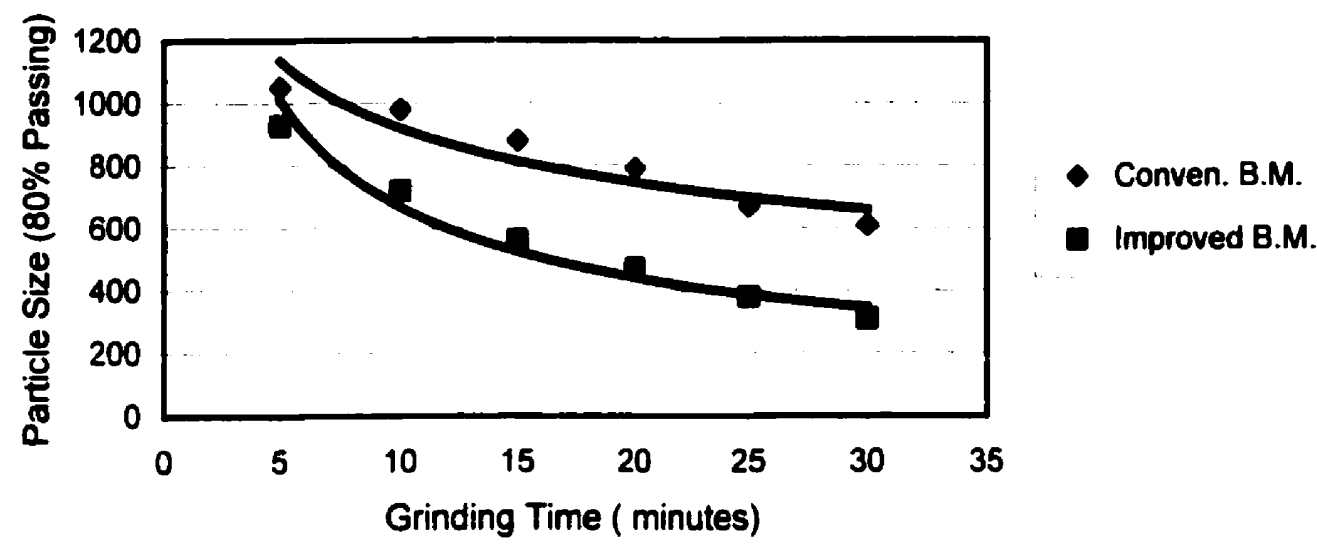

Fig 23 Particle size reduction at $87 \%$ of the critical speed, 350 grams of media, and 50 grams of sample for the both modes of operation.

Figure 24 shows the particle size reduction for the improved and the conventional ball mill at 30 minutes of grinding time, 350 grams media and $87 \%$ of the theoretical critical speed. The ability of the improved ball mill is double that of the conventional ball mill in terms of particle size reduction. 
Table 3 Comparison of particle size reduction of the improved ball mill and the conventional ball mill under the same operational conditions.

\begin{tabular}{|c|c|c|c|c|c|c|c|c|c|}
\hline \multicolumn{4}{|c|}{ Feed } & \multicolumn{3}{|c|}{ Conventional Ball Mill } & \multicolumn{3}{|c|}{ Improved Ball Mill } \\
\hline Aperture & Weight & Weight \% & $80 \%$ Passing & Weight & Weight \% & $80 \%$ Passing & Weight & Weight \% & $\begin{array}{l}80 \% \\
\text { Passing }\end{array}$ \\
\hline Microns & Grams & Feed & Feed & Grams & $\begin{array}{l}\text { Conventional } \\
\text { B.M }\end{array}$ & $\begin{array}{l}\text { Conventional } \\
\text { B.M }\end{array}$ & Grams & $\begin{array}{l}\text { Improved } \\
\text { B.M }\end{array}$ & $\begin{array}{l}\text { Improved } \\
\text { B. M }\end{array}$ \\
\hline+1700 & 0 & $\overline{0}$ & 100 & 0 & 0 & 100 & 0 & 0 & 100 \\
\hline 1180 & 10.11 & 20 & 80 & 2.02 & 4.03 & 95.97 & 1.01 & 2.02 & 97.98 \\
\hline 850 & 13.14 & 26.13 & 53.87 & 3.54 & 7.06 & 88.91 & 1.14 & 2.27 & 95.71 \\
\hline 600 & 9.35 & 18.59 & 35.28 & 5.21 & 10.39 & 78.52 & 2.43 & 4.85 & 90.86 \\
\hline 425 & 7.05 & 14.02 & 21.26 & 4.85 & 9.67 & 68.85 & 2.32 & 4.63 & 86.23 \\
\hline 300 & 5.82 & 11.57 & 9.69 & 6.96 & 13.88 & 54.97 & 3.62 & 7.23 & 79 \\
\hline 212 & 3.33 & 6.62 & 3.07 & 7.44 & 14.84 & 40.13 & 3.18 & 6.35 & 72.65 \\
\hline 150 & 1.42 & 2.82 & 0.25 & 4.63 & $\overline{9.23}$ & 30.9 & 2.99 & 5.97 & 66.68 \\
\hline 106 & 0.04 & 0.079 & & 4.87 & 9.71 & 21.19 & 6.82 & 13.62 & 53.06 \\
\hline 75 & 0.01 & 0.019 & & 3.74 & 7.46 & 13.73 & 14.97 & 29.9 & 23.16 \\
\hline 53 & & & & 4.13 & 8.24 & 5.49 & 9.29 & 18.56 & 4.6 \\
\hline-38 & & & & 254 & 5.06 & 0.43 & 2.29 & 4.57 & 0.45 \\
\hline & & & & & & & & & \\
\hline
\end{tabular}

- Cumulative weight percent of the feed is $1180 \mu \mathrm{m}$.

- Cumulative weight percent of the conventional ball mill is $610 \mu \mathrm{m}$.

- Cumulative weight of the improved ball mill is $310 \mu \mathrm{m}$. 


\section{Particle Size Reduction}

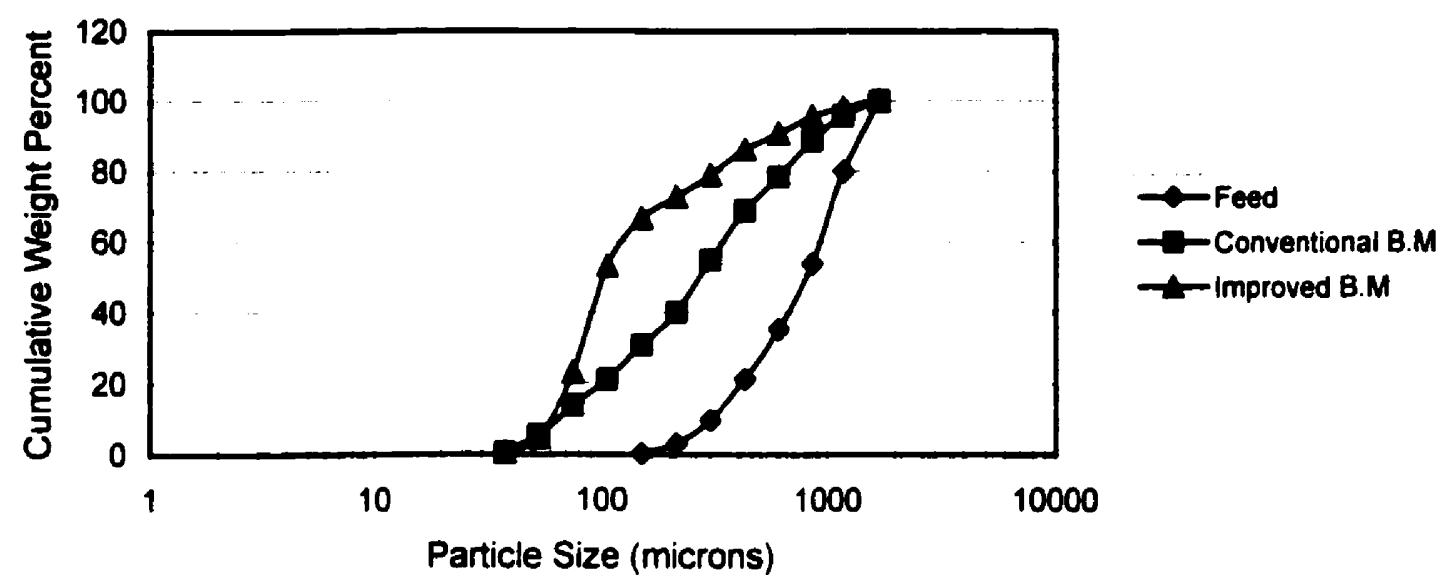

Figure 24 Particle size reduction for the both modes of operation at 30 minutes, 350 grams media, 50 grams of the sample weight and $87 \%$ of the critical speed.

\section{4-2-4 Effect of Grinding Media Loading}

Three tests have been performed to determine the effect of the media loading in the mill on the particle size reduction. In the tirst test the ratio of the grinding media to the feed particles was 1: 4 by weight, in the second one was $1: 7$ by weight, and in the third test was 1: 15 by weight. Results of these experiments are shown in the appendix A-C.

\section{4-2-4-1 350 grams media and $80 \%$ of eritical speed}

As shown in the chart of Figure 25. for 350 grams media, 50 grams of sample, and $80 \%$ of the critical speed, the constant $C$ is calculated to be 2.66 . This means that the ability of the improved ball mill to produce finer and more homogeneous product is 2.66 times the ability of the conventional ball mill under this operational condition, such calculation is shown in Table 5 and Appendix D2. 


\section{Effect of Media Loading}

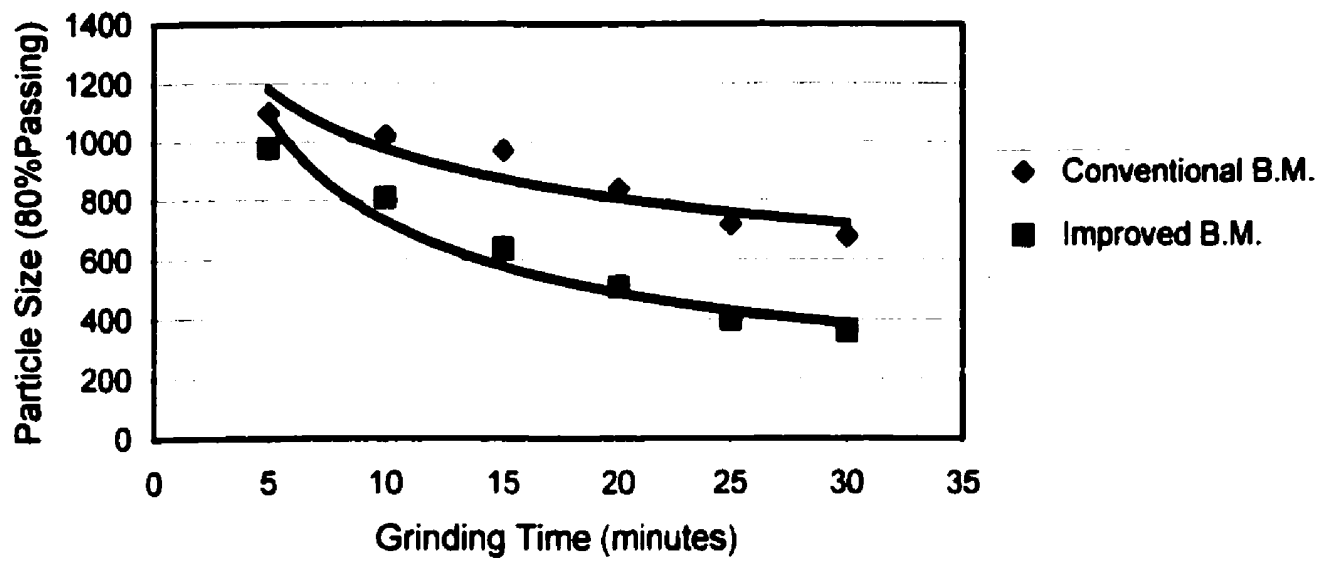

Figure 25 Effect of media loading on the grinding ability for the conventional and improved ball mills at $350 \mathrm{~g}$ media, $50 \mathrm{~g}$ sample weight, and $80 \%$ of critical speed.

\section{4-2-6 Ball Mill Grindability Test}

The procedure of testing the improved ball mill is adapted from MINE 331 Lab Manual, Courtesy of Queen's University, Mining Engineering department. A detailed procedure, including sample calculation, presented by Rene' John Deister in $1987^{[27.28 \mid}$

\section{4-2-6-1 Procedures}

1- A screen analysis was carried-out on about 250 grams of the assigned mesh size sample. A typical size distribution curve is shown in Figure 24. The feed at $80 \%$ passing size and the weight percent of the sample were calculated as in Table 3.

2- A $1000 \mathrm{ml}$ graduated cylinder was packed to the $700-\mathrm{cm} 3$ mark, shaken until compact, and then weighed. Table 4 is a typical table used in calculations, and in this case for $-65-$ mesh size. 
3- The $700-\mathrm{cm} 3$ sample was ground in the ball mill for an arbitrary 100 number of revolutions. The sample was removed from the mill and screened through a 65 -mesh screen using a Rotap machine. The removed -65 mesh material was replaced with a fresh -6 mesh feed material.

4- The net grams of -65 -mesh product (total -65 -mesh product the "in feed" -65 mesh) and the net grams per revolution were calculated. This is shown in column 6 and 7, Table 4 .

5- The "ideal potential product" was then calculated. This is the net weight of the product that must be produced for each cycle to allow the mill to run at $250 \%$ circulating load.

\section{Ideal potential product $=$ Feed Weight $/ 3.5$}

6 The ideal potential product minus the "in feed -65 mesh" for cycle 2 divided by the net grams per revolution gives the number of revolution for the next cycle.

7 The replenished sample charge was put back into the ball mill and ground for the calculated number of revolutions, column 3 .

8 The sample charge was removed, screened, and that -65 mesh was removed was again replaced. The number of revolutions for the next cycle was calculated and the material was ground again. This procedure was repeated until two or three consecutive cycles had produced products of consistent weight close to that of the ideal potential product.

The following equation was used in calculations of work index:

$$
W_{i}=\frac{44.5}{\left\{\left(P_{i}\right)^{0.23} \times(G b p)^{0.82} \times\left[\left(\frac{10}{\sqrt{P}}\right)-\frac{10}{\sqrt{F}}\right]\right\}}
$$


where $\boldsymbol{W}_{i}$ is the mill work index.

$P_{l}$ is the microns opening in sieve size tested.

Gbp is the average of the net grams of undersize produced per revolution during the last three cycles.

$P_{2}$ is the microns at which $80 \%$ of the last cycle sieve undersize product passes.

$F_{2}$ is the microns at which $80 \%$ of the new ball mill feed passes.

The value of $\boldsymbol{W}_{l}$ obtained from this equation is used to calculate the energy input to the mill by the equation:

$$
W=W_{i}\left(\frac{10}{\sqrt{P}}-\frac{10}{\sqrt{F}}\right)
$$

where $W$ is the energy input to the mill, $\mathrm{kWhr} / \mathrm{st}$

$W i$ is the work index $(\mathrm{kWhr} / \mathrm{st})$

$P$ is the square sieve size opening at which $80 \%$ of the product passes (microns).

$F$ is the square sieve size opening at which $80 \%$ of the feed passes (microns).

\section{4-2-6-2 Calculation of the Work Index}

\section{Mesh Sieve Size Opening}

$P_{1}=208$ microns $\quad P_{2}=214$ microns $\quad F_{2}=2617$ microns

Gbp $=2.330$ (average of the last three cycles)

$$
\begin{aligned}
& W_{i}=\frac{44.5}{\left\{(208)^{0.23} \times(2.33)^{0.82} \times\left[\left(\frac{10}{\sqrt{214}}-\frac{10}{\sqrt{2617}}\right)\right]\right\}} \\
& W_{i}=13.35
\end{aligned}
$$


The Work Index is not a material constant; it varies with the particle size of the material. The value of 13.35 is the Work Index for the sample used in these tests with a particle size equal to 212 microns.

\section{4-2-6-3 Bond Ball Mill Grindability Test}

Table 4 Typical calculation in the Bond ball mill circuit grindability test Sample: Limestone Feed: $14.74 \%$ minus 65 mesh

\begin{tabular}{|r|r|r|r|r|r|r|}
\hline & & \multicolumn{3}{|l|}{ Grams of minus } & \multicolumn{2}{l|}{ 65Mesh } \\
\hline Cycle No. & New Feed & Number of & In Mill & In Mill & \multicolumn{1}{l|}{ Net } & Net per \\
\hline & (Grams) & Revolution & Product & Feed & Product & Revolution \\
\hline 1 & 1286.35 & 100 & 407.19 & 189.61 & 217.58 & 2.176 \\
\hline 2 & 407.19 & 144 & 365.85 & 60.02 & 305.83 & 2.169 \\
\hline 3 & 365.85 & 145 & 377.33 & 53.93 & 323.4 & 2.23 \\
\hline 4 & 377.33 & 140 & 378.71 & 55.62 & 323.09 & 2.308 \\
\hline 5 & 378.71 & 135 & 372.12 & 55.82 & 316.3 & 2.343 \\
\hline 6 & 372.12 & 134 & 368.33 & 54.85 & 313.48 & 2.339 \\
\hline 7 & & & & & & \\
\hline
\end{tabular}

Unit volume $(700 \mathrm{ml})=1286.35$ grams in mill

Ideal Potential Product $=\mathbf{3 6 7 . 5 3}$

Average of last 3 cycles: Circulating load $=913.30$ grams or $245 \%$

Product $=2.330$ Net gram -65 mesh $/$ Rev .

$P_{1}=208$ microns $\quad P_{2}=214$ microns $\quad F_{2}=2617$ microns

$\mathrm{GbP}=2.330$ (average for the last 3 cycles)

$W_{i}=13.34927958 \cong \mathbf{1 3 . 3 5}$ 


\section{4-2-7 Time / Particle Size Relationship and Effect of Magnet}

Introducing a magnetic field to the conventional ball mill reduces the grinding time and saves energy too. Several runs of both modes of the ball mill have been performed. The test results indicate that the grinding time is not a linear relation with the particle size of the product. The grinding time / the particle size relationship has to be defined from the equation:

$$
\mathrm{W}=\mathrm{C} \frac{\text { Time }}{(\text { Weight of Sample)(Particle Size })}
$$

To determine the relationship of the grinding time to the particle size reduction, the following equations are used ${ }^{[28]}$.

$$
\begin{aligned}
& E_{N M}=W_{i}\left(\frac{10}{\sqrt{P_{80 \% N M}}}-\frac{10}{\sqrt{F_{80 \% N M}}}\right) \\
& E_{M}=W_{i}\left(\frac{10}{\sqrt{P_{80 \% M}}}-\frac{10}{\sqrt{F_{80 \% M}}}\right)
\end{aligned}
$$

where $E$ is the energy consumed in the grinding process, $\boldsymbol{W}_{\boldsymbol{i}}$ is the Bond work Index. $\mathbf{P 8 0 \%}$ is the size from which $\mathbf{8 0 \%}$ of the product passes in microns, assuming this has some valid relationship to the total surface area; $F 80 \%$ is the size which $80 \%$ of the feed passes in microns. The subscript $(N M)$ indicates that the ball mill was used in the conventional ball-milling mode. And the subscript $(\boldsymbol{M})$ indicates that the ball mill was used in the improved ball-milling mode. 
For the same grinding time, the same ball mill, the same amount of sample, the same feed size distribution, and the same grinding media, then:

$$
\begin{aligned}
& W_{i}\left(\frac{10}{\sqrt{P_{80 \% N M}}}-\frac{10}{\sqrt{F_{80 \% N M}}}\right)=W_{i}\left(\frac{10}{\sqrt{P_{80 \% M}}}-\frac{10}{\sqrt{F_{80 \% M}}}\right) \\
& \frac{E_{M}}{E_{N M}}=C=\left(\frac{\frac{10}{\sqrt{P_{80 \% M}}}-\frac{10}{\sqrt{F_{80 \% M}}}}{\frac{10}{\sqrt{P_{80 \% N M}}}-\frac{10}{\sqrt{F_{80 \% N M}}}}\right) \\
& \mathrm{E}_{\mathrm{NM}}=\mathrm{CE}_{\mathrm{M}}
\end{aligned}
$$

The constant $\mathbf{C}$ is the time difference in the grinding process.

The analysis shows that the improved ball mill saves an average of time up to 2.5 times the time required for the conventional ball mill to produce an assigned particle size. Such calculation is shown in Appendix D2.

\section{4-2-7-1 Example of Calculation}

The feed sample has a particle size equal to 1200 microns, and the product powder has a particle size equal to $\mathbf{4 7 0}$ microns, after $\mathbf{2 0}$ minutes of milling in an improved ball mill. The constant $C$ is calculated using the above method to equal 2.57. The energy consumed in the grinding process with an improved ball mill is $2.3041 \mathrm{Kwhr} / \mathrm{st}$. . When using a conventional ball mill to get the same particle size, the energy required is $2.3041 \times 2.5719=5.9259 \mathrm{kWhr} / \mathrm{st}$. 
Table 5 Calculation of the energy consumed on particle size reduction of 50 grams of sample weight at 350 grams media, and $80 \%$ of the critical speed.

\begin{tabular}{|c|c|c|c|c|c|c|c|}
\hline $\begin{array}{l}\text { Grinding } \\
\text { Time }\end{array}$ & Feed & $\begin{array}{l}\text { Prod.80\% } \\
\text { Passing } \\
\end{array}$ & $\begin{array}{l}\text { Prod.80\% } \\
\text { Passing }\end{array}$ & constant & Energy & Average & Energy \\
\hline Minutes & $80 \%$ Passing & $\begin{array}{l}\text { Conventional } \\
\text { B.M }\end{array}$ & $\begin{array}{l}\text { Improved } \\
\text { B.M }\end{array}$ & C & $\begin{array}{l}\text { Improved } \\
\text { B.M }\end{array}$ & $\mathbf{C}$ & $\begin{array}{l}\text { Conventional } \\
\text { B.M }\end{array}$ \\
\hline 5 & 1200 & 1100 & 980 & 2.3971 & 0.4106 & 2.666 & 1.0962 \\
\hline 10 & 1200 & 1020 & 810 & 2.5656 & 0.8368 & 2.666 & 2.2341 \\
\hline 15 & 1200 & 970 & 640 & 3.2902 & 1.4231 & 2.666 & 3.7993 \\
\hline 20 & 1200 & 840 & 510 & 2.7351 & 2.0576 & 2.666 & 5.4929 \\
\hline 25 & 1200 & 720 & 400 & 2.5157 & 2.8211 & 2.666 & 7.5313 \\
\hline \multirow[t]{3}{*}{30} & 1200 & 680 & 360 & 2.5143 & 3.1821 & 2.666 & 8.4952 \\
\hline & & & Total & 16.018 & & & \\
\hline & & & Average & 2.6696 & & & $37.50 \%$ \\
\hline
\end{tabular}

Effect of Magnet on Particle Size Reduction

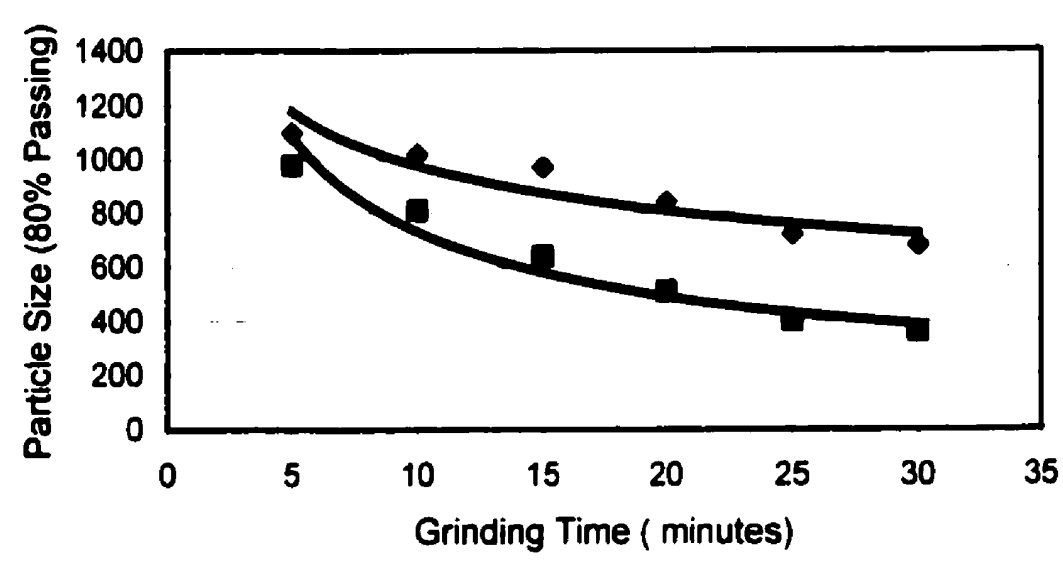

- Conventional B.M.

- improved B.M.

Figure 26 Effect of magnet on the particle size reduction at 350 grams media loading, $80 \%$ of the critical speed, and 50 grams sample.

Introducing a magnetic force to the ball mill reduces the grinding time by 2.66 of the time used in the conventional ball mill as shown in Table 5 and Figure 26 at operational condition of $350 \mathrm{grams}$ media loading, 50grams of the sample weight and $80 \%$ of the 
critical speed. The particle size $640 \mu \mathrm{m}$ was obtained after 15 minutes grinding in the improved ball mill, while the particle size of $680 \mu \mathrm{m}$ was obtained after 30 minutes grinding in the conventional ball mill.

\section{4-2-8 Energy Consumed on the Particle Size Reduction}

The "laws of energy" relating the degree of comminution to the grinding effected has produced so much controversy. Rittinger, Kick and Bond cannot all be right. It is inconceivable that the energy input can be proportional to $\mathrm{X}^{3}$ and $\mathrm{X}^{2}$ or any intermediate exponent of $\mathrm{X}$ all at the same time. All the laws are true but each applies over a limited range of particle size. The laws of energy consumed in particle size reduction are not laws of nature but laws of technology, i.e. laws of machinery operation. If the machine does not obey one or other of the laws it is an unsuitable machine. But how do we know it is unsuitable except that it violates the law. But how do we know that the law is correct? [25].

From the point of view of the practical engineer, Bond's law has much to commend it. Work Index values are available in the literature on a variety of materials. In the sieve size range, Bond's law is a particularly useful predictor of energy consumption in the grinding process.

\section{4-2-9 Particle Size / Energy}

Using Bond's law the energy consumed in the particle size reduction carried-out here is calculated. The constant $\boldsymbol{C}$ is determined for each run and the energy is calculated and compared for both ball mills. Using the improved ball mill saves an average of about $40 \%$ of the energy consumed on the particle size reduction as observed using the

conventional ball mill. Table 6 shows a comparison of the two mill types in terms of 
energy consumption and particle size reduction. Calculations of the energy consumed in the particle size reduction at different parameters are shown in appendix D2.

Table 6 Energy consumed on particle size reduction in the both modes of operation at 350grams media, 87\% of the critical speed and 50grams of sample weight.

\begin{tabular}{|r|r|r|r|r|r|r|r|}
\hline $\begin{array}{l}\text { Grinding } \\
\text { Time }\end{array}$ & $\begin{array}{l}80 \% \\
\text { passing }\end{array}$ & $\begin{array}{l}\text { Prod.80\% } \\
\text { Passing }\end{array}$ & $\begin{array}{l}\text { Prod.80\% } \\
\text { Passing }\end{array}$ & Constant & Energy & Average & \multicolumn{1}{l|}{ Energy } \\
\hline Minutes & Feed & Conven. & Improved & \multicolumn{1}{c|}{ C } & Improved & \multicolumn{1}{l|}{ C } & Conven. \\
\hline 5 & 1200 & 1050 & 930 & 1.9688 & 0.5237 & 2.4562 & 1.2864 \\
\hline 10 & 1200 & 980 & 720 & 2.7309 & 1.1213 & 2.4562 & 2.7543 \\
\hline 15 & 1200 & 880 & 560 & 2.7653 & 1.7875 & 2.4562 & 4.3905 \\
\hline 20 & 1200 & 790 & 470 & 2.5719 & 2.3041 & 2.4562 & 5.6591 \\
\hline 25 & 1200 & 670 & 380 & 2.2969 & 2.9945 & 2.4562 & 7.3552 \\
\hline 30 & 1200 & 610 & 310 & 2.4032 & 3.7284 & 2.4562 & 9.1577 \\
\hline & & Total & 14.737 & & & \\
\cline { 4 - 8 } & Average & 2.4562 & & & $40.70 \%$ \\
\hline
\end{tabular}

Figure 27 illustrates the particle size reduction and the energy consumed for each particle size produced during the grinding process. The ball mill operated at $87 \%$ of the critical speed, $\mathbf{3 5 0}$ grams of media and $\mathbf{5 0}$ grams of sample.

\section{Particle Size / Energy}

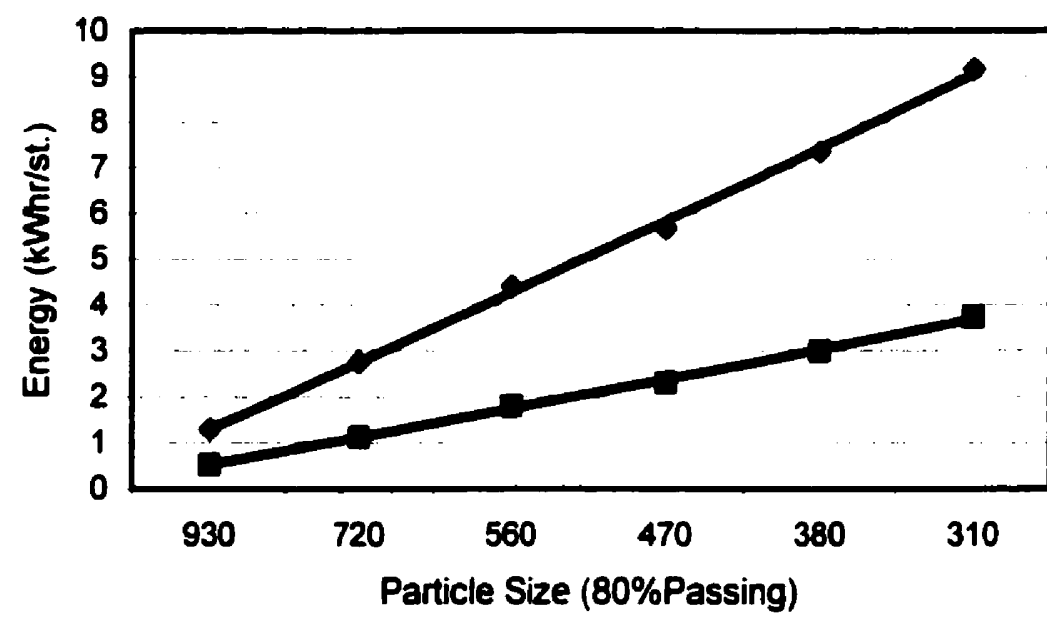

- Conventional B.M.

- Improved B.M.

Figure 27 Energy consumed on the particle size reduction at $350 \mathrm{~g}$ media, $87 \%$ of the critical speed and $50 \mathrm{~g}$ of sample weight for conventional and improved ball mill. 
Energy consumed on particle size reduction with 750 grams media loading, 87\% of the critical speed, and 50 grams weight of sample is shown in Table 7.

Table 7 Illustrates the energy consumed on particle size reduction at 750 grams media, $87 \%$ of the critical speed and 50grams of the sample weight for the improved and conventional ball mill.

\begin{tabular}{|c|c|c|c|c|c|c|c|}
\hline $\begin{array}{l}\text { Grinding } \\
\text { Time }\end{array}$ & \begin{tabular}{|l|}
$80 \%$ \\
Passing \\
\end{tabular} & $\begin{array}{l}\text { Prod. } 80 \% \\
\text { Passing }\end{array}$ & $\begin{array}{l}\text { Prod. } 80 \% \\
\text { Passing } \\
\end{array}$ & Constant & Energy & Average & Energy \\
\hline minutes & Feed & Conven. & Improved & C & Improved & C & Conven. \\
\hline 5 & 1200 & 1040 & 910 & 2.0001 & 0.5716 & 2.4039 & 1.3741 \\
\hline 10 & 1200 & 960 & 700 & 2.6207 & 1.1919 & 2.4039 & 2.8653 \\
\hline 15 & 1200 & 860 & 520 & 2.8642 & 2.0004 & 2.4039 & 4.8089 \\
\hline 20 & 1200 & 740 & 420 & 2.5247 & 2.6602 & 2.4039 & 6.3949 \\
\hline 25 & 1200 & 620 & 340 & 2.2461 & 3.3861 & 2.4039 & 8.1401 \\
\hline \multirow[t]{3}{*}{30} & 1200 & 550 & 290 & 2.1677 & 3.9855 & 2.4039 & 9.5807 \\
\hline & & & Total & 14.4235 & & & \\
\hline & & & Average & 2.4039 & & & $41.59 \%$ \\
\hline
\end{tabular}

\section{Particle Size Reduction I Energy}

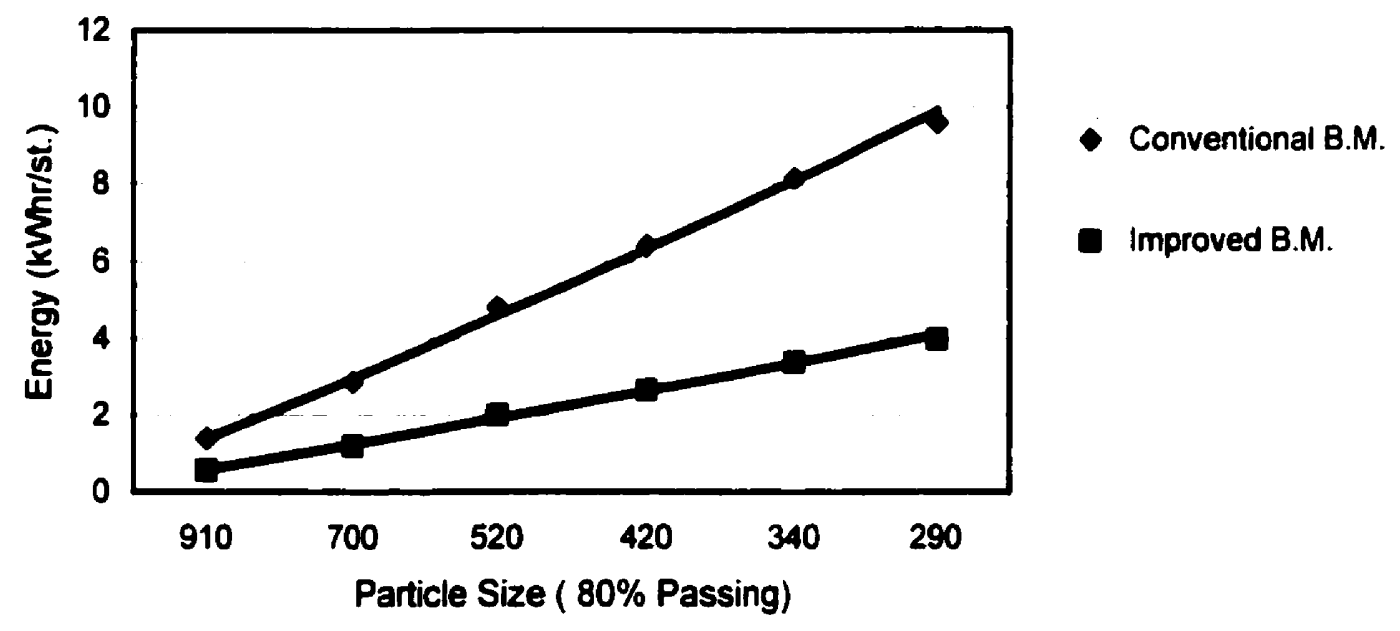

Figure 28 Comparison of the energy consumed on the particle size reduction of an improved and a conventional ball mill at $\mathbf{7 5 0}$ grams, $87 \%$ of the critical speed, and 50 grams sample. 


\section{4-2-10 Optimum Condition of Operation}

Many factors have a significant effect on the grinding efficiency and the energy consumed in the grinding process, such as the effect of sample weight, the grinding media, and the rotational speed. In order to determine the optimum condition for running the improved ball mill, the effects of these factors were tested.

\section{4-2-10-1 Effect of the Sample Weight}

Five different values of the sample weight as shown in Table 8 were used for this test. The media weight was 350 grams, and the rotational speed was $87 \%$ of the theoretical critical speed. The grinding time was 20 minutes. The optimum weight of sample for the improved ball mill was $\mathbf{5 0}$ grams as is illustrated in Table 8 and Figure 29.

Table 8 Effect of the sample weight on the grinding ability in the improved and conventional ball mill.

\begin{tabular}{|l|l|l|}
\hline $\begin{array}{l}\text { Sample weight } \\
\text { (grams) }\end{array}$ & $\begin{array}{l}\text { P. size ( 80\% Passing) } \\
\text { Conventional B.M. }\end{array}$ & $\begin{array}{l}\text { P. size ( 80\% Passing) } \\
\text { Improved B.M. }\end{array}$ \\
\hline 25 & 970 & 280 \\
\hline 35 & 915 & 370 \\
\hline 50 & 1030 & 470 \\
\hline 75 & 1050 & 520 \\
\hline 100 & 1080 & 980 \\
\hline
\end{tabular}




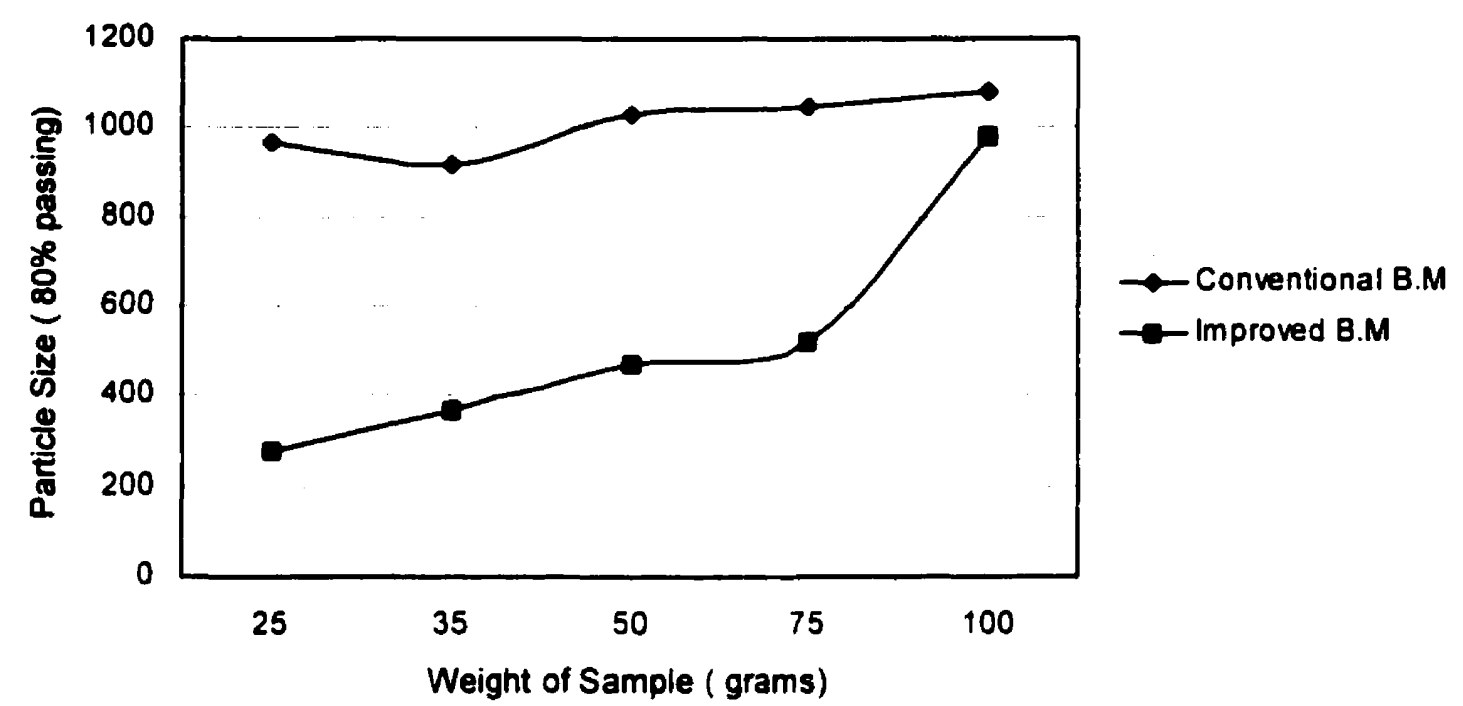

Figure 29 Effect of weight of sample on the grinding ability in the improved ball mill and the conventional ball mill with $350 \mathrm{~g}$ of media loading, $87 \%$ critical speed and 20 minutes grinding.

\section{4-2-10-2 Effect of Media Loading}

Four different weights of the media loading in the ball mill have been tested as shown in table 9. The rotational speed is $87 \%$ of the critical speed, the grinding time is 20 minutes and 50 grams weight of sample. The best weight of media was found to be the 350 grams. The optimum media weight of operating the improved ball mill as shown in Table 9 and Figure 30 was also 350grams. The detailed analyses are in Appendix El.

Table 9 Effect of the media loading on the particle size reduction in the improved and conventional ball mill operating under the same conditions.

\begin{tabular}{|l|l|l|}
\hline Media Loading (grams) & $\begin{array}{l}\text { P. Size ( 80\% Passing) } \\
\text { Conventional B.M. }\end{array}$ & $\begin{array}{l}\text { P. Size ( 80\% Passing) } \\
\text { Improved B.M. }\end{array}$ \\
\hline 175 & 990 & 980 \\
\hline 350 & 1030 & 470 \\
\hline 500 & 995 & 370 \\
\hline 750 & 980 & 300 \\
\hline
\end{tabular}


Media Loading

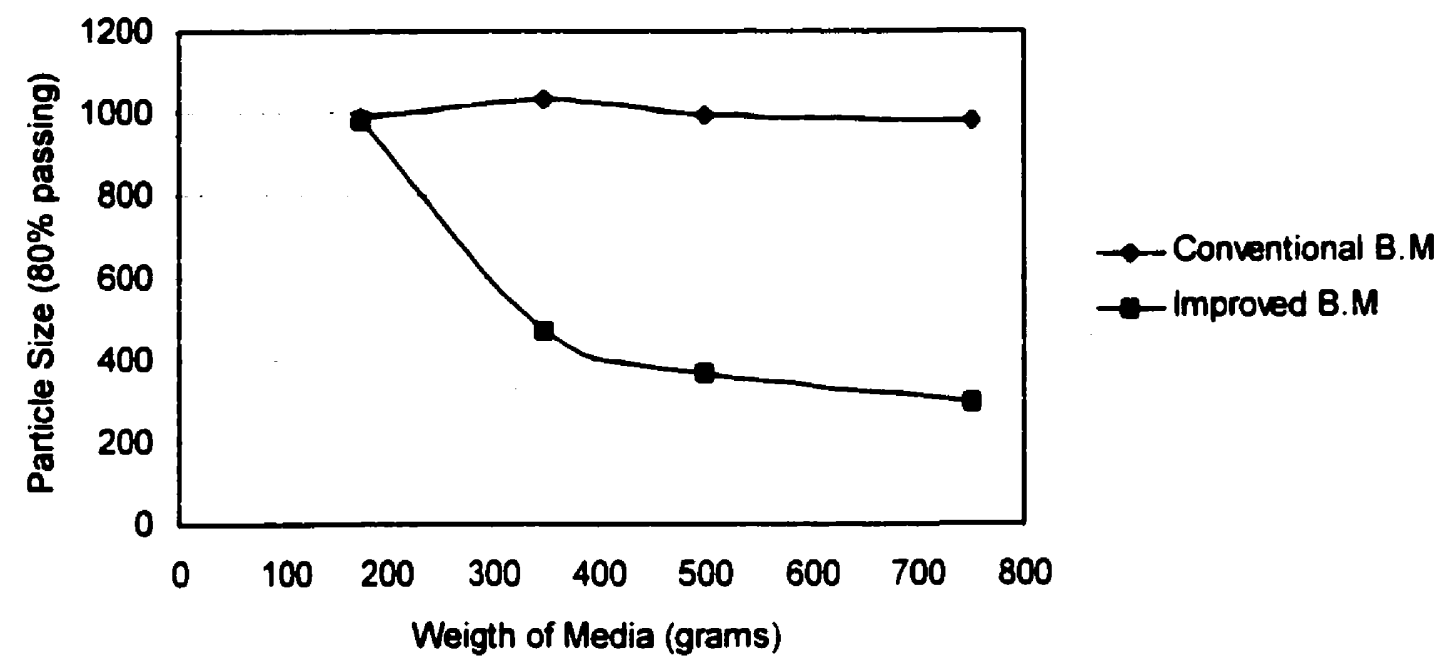

Figure 30 Effect of media loading on the grinding ability of the improved ball mill and conventional ball mill with $50 \mathrm{~g}$ sample, $87 \%$ of the critical speed and 20 minutes of grinding.

\section{4-2-10-3 Effect of Rotational Speed}

Five different rotational speeds as shown in Table 10 are tested to determine the optimum speed at which the improved ball mill can be run. The sample weight is $\mathbf{5 0}$ grams, the weight of media is 350 grams, and the grinding time is 20 minutes. The improved ball mill has found to be run at $87 \%$ of the critical speed as an optimum rotational speed as illustrated in Figure 31 and Table 10. More details on these analyses are shown in Appendix El. 
Table 10 Effect of rotational speed on the grinding ability in the improved and conventional ball mill operating under the same operational conditions.

\begin{tabular}{|l|l|l|}
\hline Rotational Speed (\% of the C. S.) & $\begin{array}{l}\text { P. Size ( 80\% Passing) } \\
\text { Conventional B.M. }\end{array}$ & $\begin{array}{l}\text { P. Size ( 80\% Passing) } \\
\text { Improved B.M. }\end{array}$ \\
\hline 70 & 930 & 800 \\
\hline 80 & 840 & 510 \\
\hline 87 & 790 & 470 \\
\hline 95 & 860 & 720 \\
\hline 98 & 920 & 830 \\
\hline
\end{tabular}

\section{Rotational speed}

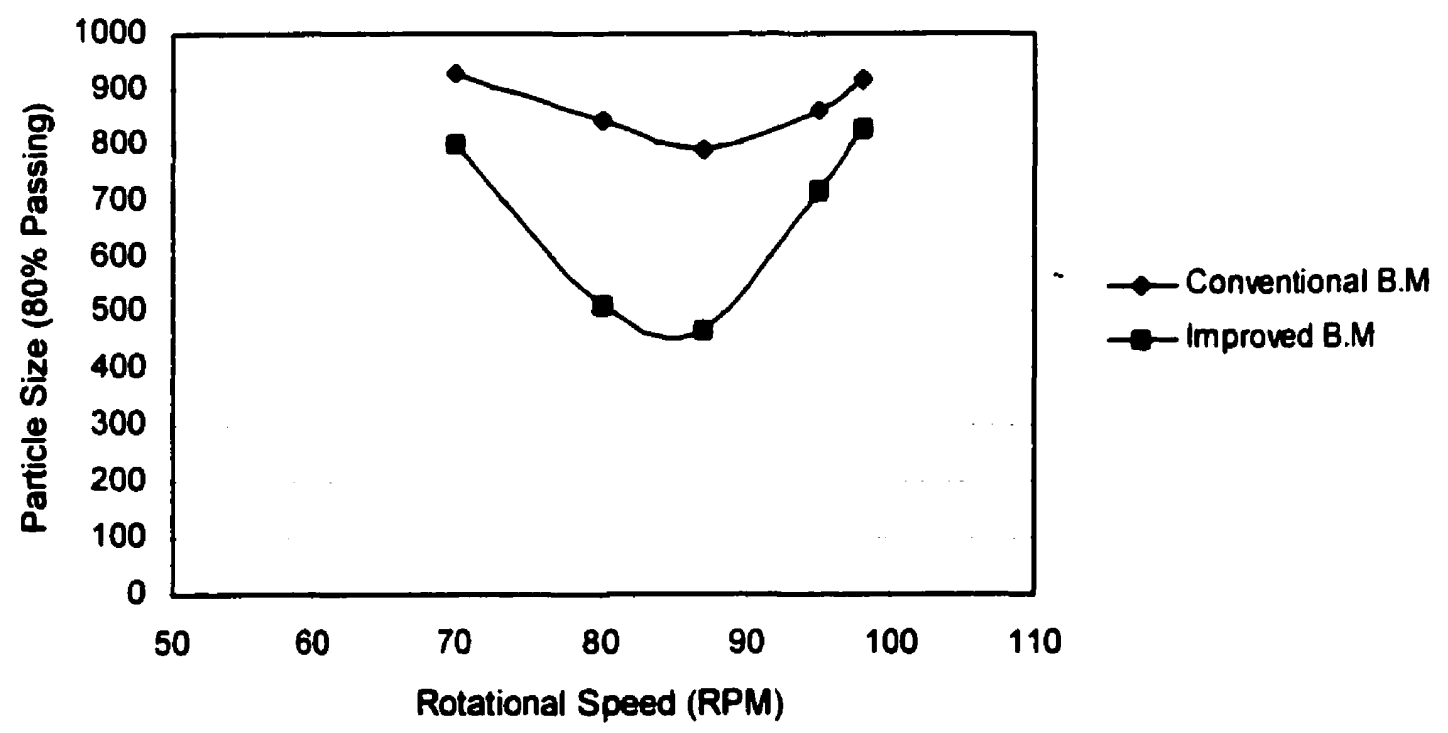

Figure 31 Effect of the rotational speed on the grinding ability of the improved and conventional ball mill at $350 \mathrm{~g}$ media, $50 \mathrm{~g}$ of sample and 20 minutes of grinding time. 


\section{4-3 Discussion}

The patterns of the ball movement that can be achieved using the improved ball mill are shown in Figure 19. By adjusting the magnetic field or the rotational speed of the mill, different modes of operation are obtained. The three modes of operation of the conventional ball mill, cascading, cataracting, and hurricane have been replaced by the new patterns of the ball movement in the improved ball mill. In general, the modes of operation of the improved ball mill can be classified into three modes of operation, depending on the position of the magnet(s) around the mill and the rotational speed of the mill chamber; They are (i) high-energy mode or the high rotational speed; (ii) the low energy mode or the low rotational speed; and (iii) the intermediate energy mode or the intermediate rotational speed, as shown in Figure 19.

The improved ball mill has been tested under different conditions of operation and the results are compared with those of the conventional ball mill under the same condition and parameters. Table 3 shows that the ability of the improved ball mill is better in comparison with the ability of the conventional ball mill in terms of particle size reduction. Most of the grinding in the improved ball mill has been done at the expense of the coarser particles due to the cataracting action. Also, a narrow range of particle size is obtained. Grinding in the conventional ball mill is done at the expense of the smaller particle size, because of the abrasion due to the cascading action.

Introducing a magnetic field into the conventional ball improves the ability of the grinding process at different values of the rotational speed. From the chart of comparison, at a rotational speed of $87 \%$ of the theoretical critical speed, Figure 22 , the 
ability of the improved ball mill is 2.45 times the ability of the conventional ball mill at this value of rotational speed.

Several runs of the improved ball mill and the conventional ball mill have been performed with different values of media loading to determine the effect of the media loading on the efficiency of the grinding process. Figure 25 shows the effect of media loading on the grinding ability for the improved and conventional ball mills. For a 350 grams media loading, rotational speed of $\mathbf{8 0 \%}$ of the theoretical critical speed and with 50 grams of sample weight, the ability of the improved ball mill is 2.66 times the ability of the conventional ball mill.

As stated above, applying a magnetic field to the conventional ball reduces the grinding time. Table 5 shows that the introduction of a magnetic field to the ball mill reduces the grinding time by a factor of 2.66 . A particle size of $640 \mu \mathrm{m}$ is obtained after 15 minutes of grinding in the improved ball mill, while the particle size of $680 \mu \mathrm{m}$ requires 30 minutes of grinding in the conventional ball mill at $80 \%$ of the critical speed, 350 grams of media loading and 50 grams of sample weight. Since the grinding time is reduced, the energy consumed in the particle size reduction process is also reduced, and with the same factor. Table 6 illustrates calculations of the particle size reduction and the energy consumed for each particle size produced during the grinding process. The ball mills operated at a rotational speed of $87 \%$ of the critical speed, 350 grams of media loading and 50 grams of sample weight. The improved ball mill saves $40.7 \%$ of the energy consumed by the conventional ball mill under these operational conditions. 
The optimum condition of operating the improved ball mill has been tested in terms of weight of sample, the weight of media and the rotational speed. The optimum condition of running the improved ball mill has been found to be 50 grams of sample weight, 350 grams of media loading and the rotational speed is $87 \%$ of the theoretical critical speed.

\section{4-4 Physical Properties of the Grinding Product}

A qualitative comparison of the ground product of the grinding process of the conventional and improved ball mills in terms of the physical properties is summarized as follow:

\section{4-4-1 Conventional Ball Mill}

1- Powder size distribution does not change drastically.

2- Both powder particles and welded layers are relatively soft and ductile.

3. In conventional ball milling, the proportion of powder welded to the ball increases to its maximum value toward the end of the grinding period.

4- The product forms an angle of repose.

5- Strong clustering, low homogeneity product is produced.

\section{4-4-2 Advanced Ball Mill}

1- A sharp decrease in the amount of coarse particles is observed and the product particles bind towards more equiaxed dimensions.

2- Improved ball milling has strong disintegration and powder blending action.

3- Particles are spherical and uniform.

4- The use of the improved ball mill can provide control of particle size and shape. 


\section{V-Conclusion}

1- An improved ball mill has been designed, built and tested. The Nicol Hall-built ball mill has shown its ability to produce a finer and more homogeneous particle size than that produced by the conventional ball mill.

2- The special feature of this milling device is that ball movement is controllable and reproducible. By adjusting the magnet position or the rotational speed of the mill chamber, several modes of operation are obtained.

3- Under the same operating conditions, the advanced ball mill reduced the grinding energy by about $40 \%$ of the energy consumed to achieve an equivalent particle size reduction using the conventional ball mill.

4- The improved ball mill reduces the time of the grinding process to get an assigned particle size to half of that of the conventional ball mill. At a rotational speed $87 \%$ of the critical speed, and with 350 grams of media loading and 50 grams of sample weight, the grinding time has been reduced by a tactor of 2.45 in comparison with that of the conventional ball mill under the same operational conditions.

5- The optimum condition of operating the improved ball mill has been determined in terms of the weight of sample, the weight of media loading and the rotational speed. It is found to be: $\mathbf{5 0}$ grams of the sample weight, $87 \%$ of the critical speed and 350 grams of the media weight loading in order to achieve a homogeneous particle size distribution.

6- Since the energy consumption in the grinding process is the main cost of the whole operation, the use of the improved ball mill in the mineral industry could reduce the 
cost of extraction in terms of grinding time and the consumption of energy on the grinding process.

7- In the conventional ball milling, the liners are used to raise up the charge and the media to a certain level to create cataracting action. The liners are made from a hard material to resist wear and damage. These materials are expensive and their replacement from time to time increases the cost of operating these machines. Introducing the magnetic forces may eliminate the liners in the conventional ball mill or permit the use of a cheaper liner. This would reduce the installation cost of this kind of machine, and the grinding quality would be better and less expensive.

8- The idea of controlling the ball movement in a ball mill is simple, but it has opened up many new possibilities at a time when high technology requires new materials with specific properties.

9- Using the improved ball mill in performing mechanical alloying could modify the milled alloy structure or induce solid-state reactions lead to the synthesis of new alloys or components.

10-Advanced ball milling can produce powders with good properties and uniform shapes. Many existing products could be improved by this process, which can reproducibly yield either fine particle products or particles with very uniform geometry. This kind of performance could reduce the cost of efticient grinding of particularly fine particles down to nanometre scale and also reduce mill wear.

11-It is to be anticipated that the use of improved ball mill in mechanical alloying could overcome many current difficulties in producing unusual materials, some of these could be synthesized quickly and economically by solid-solid, solid-liquid 
and/or solid gas reactions. Materials include super-hard materials such metal carbides, advanced magnetic materials and the important electronic materials such as amorphous silicon.

\section{VI-Recommendations for Future Work}

The improved ball mill, "Model l" has been designed, constructed and tested using a commercial ore. The ability of the improved ball mill has been determined in comparison with the conventional ball mill in terms of the particle size reduction, the grinding time and the energy consumed in the particle size reduction. The mode of operation of the improved ball mill depends on the results desired. By repositioning the magnets and/or reducing the rotational speed of the ball mill, different modes of operation may be obtained. The improved ball mill can be used to perform mechanical alloying of materials of interest. The design of the improved ball mill, "Model 2" is such as to control both the milling atmosphere and its temperature at different volumes of the ball mill chamber. Thus it will be possible to produce carbides, nitrides, etc...at relatively low temperature. Also, many existing products could be improved by processing them in the improved ball mill to yield tine products or particles. It is intended to explore the production of novel and difficuit-to-make materials by more closely controlling the mechanical alloying process using the improved ball mill. 


\section{VII-References}

1- Austin, L.G. and Likie, P.T., Powder Technology, 5, 1972, 215-222.

2- Austin, L. G., et al, Process Engineering of Size Reduction: Ball Milling, SME-AIME, New York, 1972.

3- Calka, A. and Radlinski, A.P., Mat. Sci. and Eng., A 134, 1991, 1350-1353.

4- Austin, L.G., Process Engineering of Size Reduction: Ball Milling, AIME, 1984, 1-60 and $79-117$.

5- AMS Committee on Milling, Metals Handbook, 7, 56-70.

6- George Charles Lowrisen, Crushing and Grinding: The Size Reduction of Solid Materials, Butterworths, London, 1974, 225-254.

7- Benjamin, J.S., Scientific American, 1970, 234, 40-48

8- Mishra, B.K. and Rajamani, R.K., Comminution Theory and Particles, Editor S. Komar Kawatra, Soc. for Mining, Metall. and Exploration, Inc. Littleton co., 1992, 427-440.

9- Gaudin, A. M., Principles of Mineral Dressing, Mcgraw-Hill, London, 1939, 92-140

10-Rose, H. E., Sullivan, R. M. E.. A Treastise on the Internal Mechanics of Ball. Tube and Rod Mills, Constable, London, 1958, 35-67.

11-Hukki, R. T., Trans. Am. Inst. Mech. Eng., 1958, 581.

12-Maurice, D. R. and Courtney, T. H., Metall. Trans., 21A, 1990, 289.

13-Kuhn,W.E., Modern Developments in Powder Metallurgy, vol.12, Metal Powder Industries Federation, Priceton, NJ, 1980, 195-218.

14- Petal, A.N. and Huhn, W.E., Modern Development in Powder Metallurgy, Volume 13, Metal Powder Industries Federation, Priceton, NJ, 1980, 27-50.

15-Takova, K., Developments in Mineral Processing, Mechanical Activation of Minerals, Volume 11, Elsevier, 1989, 70-108.

16- Davice, R.M., McDermont and C. C. Koch, Metall. Trans., 19A, 1988, 2867.

17- Hashimoto, H. and Watanabe, R., Materials Science Forum, 88-90, 1992, 89-96.

18- Benjamin, J.S., New Materials by Mechanical Alloying Techniques,Verlag,1988,3-18

19- Wills, B.A., Mineral Processing Technology, 3rd Edition, Pergamon Press, New York, $1985,182-228$.

20-Charles, R.J., Trans.A.I.M.E, 204, 1957, 80.

21-Benjamin, S.J. and Volin, T.E., Met. Trans., 5, 1974, 1929. 
22-Arthur, F. Taggart, Handbook of Mineral Dressing, Ores and Industries Minerals, John willy \& Sons Inc., New York, 1945.

23-Richard H. Bozorth, Ferromagnetism, D. Van Nostrand Company, Canada, 1967, P $838-861$

24-K.J. Standley, Oxide Magnetic Materials, Clarenden Press, Oxford, 1972, P 1-6

25- Comminution, A Report by the Institute of Chemical Engineers, Edited by V.C. Marshall, I. Chem. E. Services, London 1975.

26- Bond, F.C., The Third Theory of Comminution, Trans., AIME, vol.193, P.484, 1952

27- Deister, J. R., Engineering and Mining Journal, Feb. 1987, P 42.

28- Yen, W.T., Physical Methods of Mineral Extraction, Lab. Manual, Kingston, Dept. of Mining engineering, 1997. 


\section{VIII-Appendix}

Note: "The complete Appendix is on the attached diskette, a sample of the calculations and analysis is provided as a hard copy in the following pages."

\section{Conventional Ball Mill Tests}

- 200 grams media and $70 \%$ of the critical speed Al

- 200 grams media and $80 \%$ of the critical speed A2

- 200 grams media and $87 \%$ of the critical speed A3

- 350 grams media and $70 \%$ of the critical speed B1

- 350grams media and $80 \%$ of the critical speed B2

- 350grams media and $87 \%$ of the critical speed B3

- 750gramsmedia and $70 \%$ of the critical speed $\mathrm{Cl}$

- 750gramsmedia and $80 \%$ of the critical speed C2

- 750gramsmedia and $87 \%$ of the critical speed C3

\section{Improved Ball Mill Tests}

- 200 grams media and $70 \%$ of the critical speed $\mathrm{Al}$

- 200 grams media and $80 \%$ of the critical speed A2

- 200 grams media and $87 \%$ of the critical speed A3

- 350 grams media and $70 \%$ of the critical speed B1

- 350grams media and $80 \%$ of the critical speed B2

- 350grams media and $87 \%$ of the critical speed B3

- 750gramsmedia and $70 \%$ of the critical speed $\mathrm{Cl}$

- 750gramsmedia and $80 \%$ of the critical speed $\quad$ C2

- 750 gramsmedia and $87 \%$ of the critical speed C3

\section{Effect of the Sample Weight on the Grinding Efficiency}

\section{Conventional ball mill:}

- $25 \mathrm{~g}$ sample at $350 \mathrm{~g}$ media, $87 \%$ of the critical speed and 20 minutes run D1

- $35 \mathrm{~g}$ sample at $350 \mathrm{~g}$ media, $87 \%$ of the critical speed and 20 minutes run D1

- $50 \mathrm{~g}$ sample at $350 \mathrm{~g}$ media, $87 \%$ of the critical speed and 20 minutes run D1 
- $75 \mathrm{~g}$ sample at $350 \mathrm{~g}$ media, $87 \%$ of the critical speed and 20 minutes run

- $100 \mathrm{~g}$ sample at $350 \mathrm{~g}$ media, $87 \%$ of the critical speed and 20 minutes run

\section{Improved Ball Mill}

- $25 \mathrm{~g}$ sample at $350 \mathrm{~g}$ media, $87 \%$ of the critical speed and 20 minutes run

- $35 \mathrm{~g}$ sample at $350 \mathrm{~g}$ media, $87 \%$ of the critical speed and 20 minutes run

- $50 \mathrm{~g}$ sample at $350 \mathrm{~g}$ media, $87 \%$ of the critical speed and 20 minutes run

- $75 \mathrm{~g}$ sample at $350 \mathrm{~g}$ media, $87 \%$ of the critical speed and 20 minutes run

- $100 \mathrm{~g}$ sample at $350 \mathrm{~g}$ media. $87 \%$ of the critical speed and 20 minutes run

- Calculation of the Constant C

D2

- Effect of media loading on the grinding process, "comparison"

- Effect of rotational speed on the grinding process, "comparison"

D2

- Effect of the magnetic force on the grinding process

- Particle size/ time analysis El

- Optimum condition of the grinding process El

- Effect of media loading on the grinding process E2

- Effect of rotational speed on the grinding process E2 


\section{Apendix A1}

Improved Ball Mill Mode

20 minutes, $200 \mathrm{~g}$ media, $70 \% \mathrm{CS}$, Magnet

\begin{tabular}{|c|c|c|c|c|c|c|}
\hline \multicolumn{7}{|c|}{ With Magnet Configuration } \\
\hline \multicolumn{3}{|c|}{ Before Grinding } & \multirow[b]{2}{*}{ Cum.\% F } & \multicolumn{2}{|c|}{ After grinding } & \\
\hline Aperture & Weight & Weight $\%$ & & Weight & Weight \% & Cum.\% F \\
\hline 1700 & 0 & & 100 & & & 100 \\
\hline 1180 & 6.07 & 11.99 & 88.01 & 4.54 & 9.1 & 90.9 \\
\hline 850 & 9.52 & 18.81 & 69.2 & 5.09 & 10.21 & 80.69 \\
\hline 600 & 9.57 & 18.91 & 50.29 & 3.49 & 6.99 & 73.7 \\
\hline 425 & 9.11 & 18 & 32.29 & 2.42 & 4.85 & 68.85 \\
\hline 300 & 7.02 & 13.87 & 18.42 & 2.38 & 4.77 & 64.08 \\
\hline 212 & 7.3 & $\overline{14.42}$ & 4 & 2.09 & 4.19 & 59.89 \\
\hline 150 & 1.63 & 3.22 & 0.78 & 1.7 & 3.41 & 56.48 \\
\hline 106 & 0.24 & 0.474 & 0.306 & 12.57 & 25.21 & 31.27 \\
\hline 75 & 0.08 & 0.158 & 0.148 & 11.69 & 23.44 & 7.83 \\
\hline 53 & 0.06 & 0.118 & 0.03 & 3.15 & 6.31 & 1.52 \\
\hline 38 & 0 & & & 0.74 & 1.48 & 0.04 \\
\hline Pan & 0 & & & & & \\
\hline Total & 50.6 & & & 49.86 & & \\
\hline
\end{tabular}

20 minutes,200g media, 70\%CS, Magnet

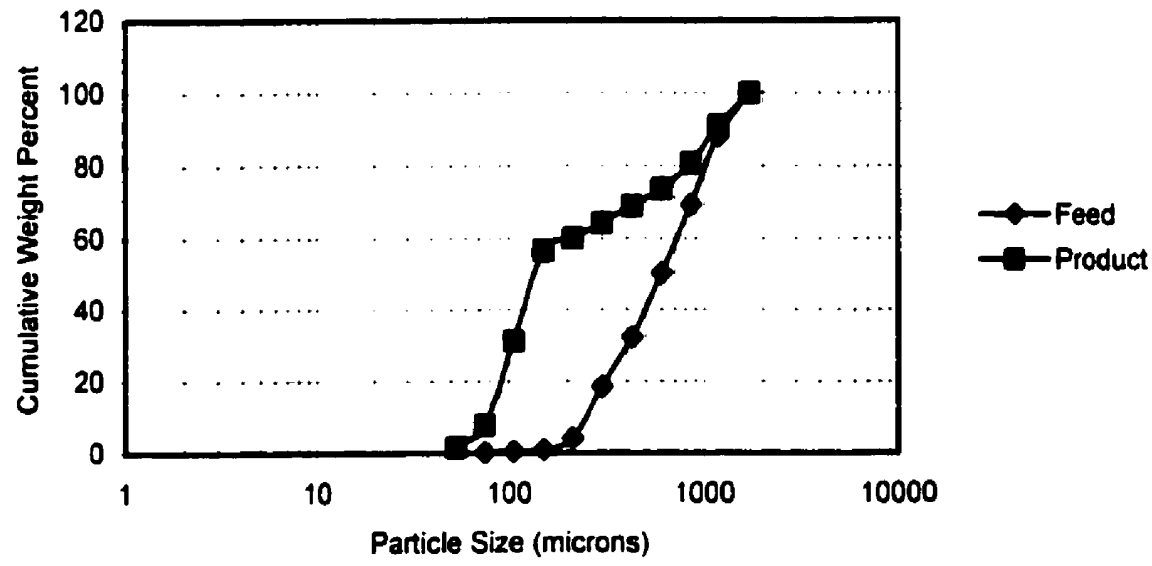




\section{Apendix A1}

\section{Conventional ball Mill}

$200 \mathrm{~g}$ media, 20 minutes, 70\%CS, No magnet

\begin{tabular}{|r|r|r|r|r|r|r|}
\hline \multicolumn{7}{|c|}{ Without Magnet Configuration } \\
\hline \multicolumn{3}{|c|}{ Before Grinding } & \multicolumn{3}{|c|}{ After grinding } & \\
\hline Aperture & Weight & Weight \% & Cum.\% F & Weight & Weight \% & Cum.\% F \\
\hline 1700 & 0 & & 100 & 0 & & 100 \\
\hline 1180 & 12.2 & 24.129 & 75.871 & 5.44 & 10.858 & 89.142 \\
\hline 850 & 14.48 & 28.639 & 47.232 & 6.67 & 13.133 & 76.009 \\
\hline 600 & 8.98 & 17.761 & 29.471 & 3.58 & 7.145 & 68.864 \\
\hline 425 & 6.61 & 13.073 & 16.398 & 1.78 & 3.552 & 65.312 \\
\hline 300 & 4.34 & 8.583 & 7.989 & 2.02 & 4.031 & 61.281 \\
\hline 212 & 3.48 & 6.882 & 1.107 & 0.59 & 1.177 & 60.104 \\
\hline 150 & 0.52 & 1.028 & 0.079 & 1.26 & 2.514 & 57.59 \\
\hline 106 & 0.04 & 0.079 & & 10.37 & 20.698 & 36.892 \\
\hline 75 & & & & 15.54 & 31.017 & 5.875 \\
\hline 53 & & & & 2.69 & 5.369 & 0.506 \\
\hline 38 & & & & 0.14 & 0.279 & 0.227 \\
\hline Pan & & & & 0.02 & 0.039 & 0.188 \\
\hline Total & 50.56 & & & 50.1 & & \\
\hline
\end{tabular}

200 g media, 20minutes, $70 \%$ CS, No magnet

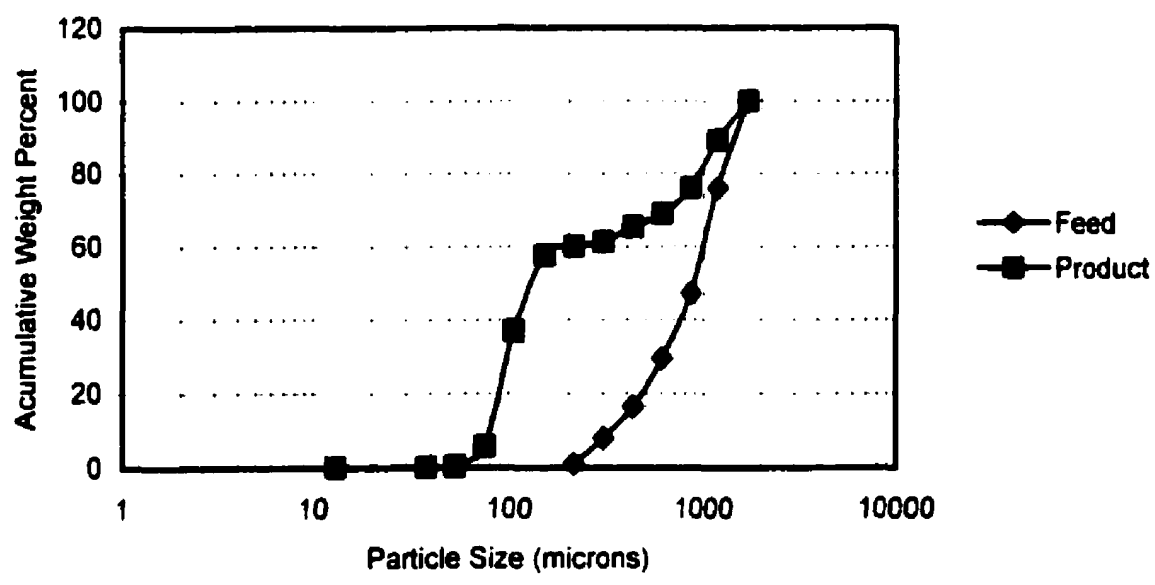




\section{Appendix A2}

Improved Ball Mill

$200 g$ modia, 20 minutes, $80 \%$ CS, Magnet

\begin{tabular}{|c|c|c|c|c|c|c|}
\hline \multirow{3}{*}{\begin{tabular}{|l|} 
Aperture \\
microns
\end{tabular}} & \multicolumn{5}{|c|}{ Grinding with magnet Configuration } & \multirow[b]{3}{*}{ Cum.\% F } \\
\hline & \multicolumn{2}{|c|}{ Bofore Grinding } & & \multicolumn{2}{|c|}{ After Grinding } & \\
\hline & Weight & Weight $\%$ & Cum.\%F & Weight & Weight \% & \\
\hline 1700 & 0 & 0 & 100 & 0 & 0 & 100 \\
\hline 1180 & 10.2 & 20.31 & 79.3 & 2.07 & 4.12 & 95.88 \\
\hline 850 & 13.51 & 26.9 & 52.8 & 3.11 & 6.2 & 89.68 \\
\hline 600 & 9.21 & 18.34 & 34.46 & 4.61 & 9.19 & 80.49 \\
\hline 425 & 7.16 & 14.26 & 20.2 & 5.84 & 11.65 & 68.84 \\
\hline 300 & 5.45 & 10.85 & 9.35 & 6.45 & 12.86 & 55.98 \\
\hline$\overline{212}$ & 4.05 & 8.06 & 1.27 & 7.19 & 14.34 & 41.64 \\
\hline 150 & 0.64 & 1.27 & 0 & 3.94 & 7.86 & 33.78 \\
\hline 106 & 0 & 0 & & 3.54 & 7.06 & 26.72 \\
\hline 75 & 0 & 0 & & 2.63 & 5.25 & 21.47 \\
\hline 53 & 0 & 0 & & 6.01 & 11.98 & 9.49 \\
\hline 38 & 0 & 0 & & 4.59 & 9.15 & 0.32 \\
\hline Pan & 0 & 0 & & 0.16 & 0.32 & 0 \\
\hline Total & 50.22 & & & 50.14 & & \\
\hline
\end{tabular}

$200 \mathrm{~g}$ media, 20 minutes, $80 \%$ CS, Magnot

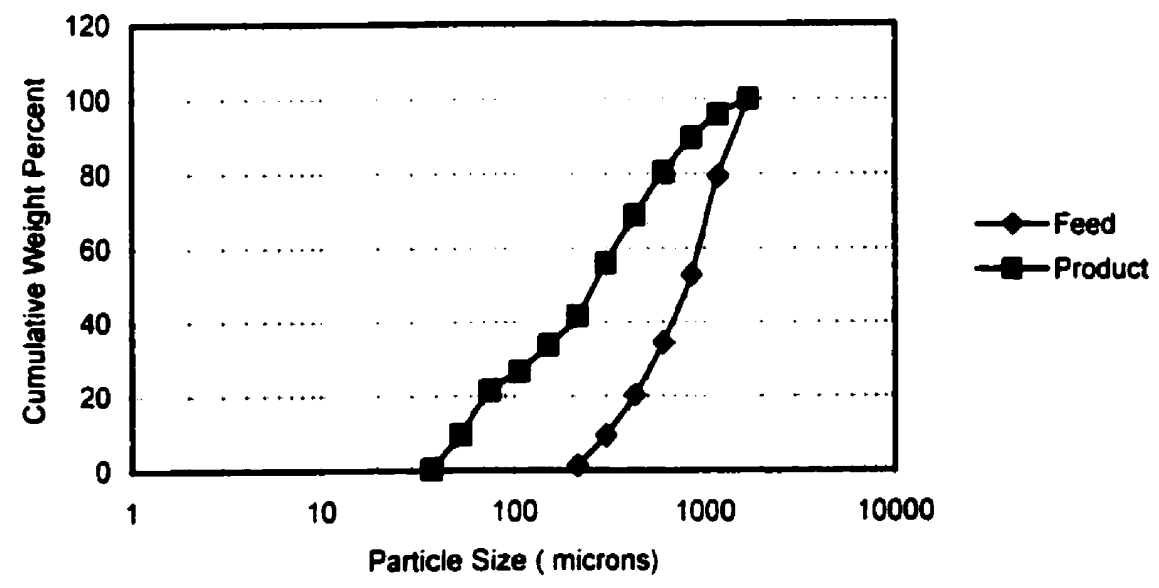




\section{Appendix A2}

\section{Conventional Ball Mill}

$200 \mathrm{~g}$ media, 20 minutes, $80 \%$ CS, No magnet

\begin{tabular}{|c|c|c|c|c|c|c|}
\hline & Grinding & without & magnet & & & \\
\hline Aperture & Bofore Gr & inding & & After Grin & ding & \\
\hline microns & Weight & Weight\% & Cum.\%F & Weight & Weight\% & Cum.\%F \\
\hline 1700 & $\overline{0}$ & & 100 & $\overline{0}$ & & 100 \\
\hline 1180 & 9.42 & 18.68 & 81.32 & 5.83 & 11.65 & 88.35 \\
\hline 850 & 11.35 & 22.51 & 58.81 & 6.03 & 12.05 & 76.3 \\
\hline 600 & 9.68 & 19.2 & 39.61 & 2.17 & 4.33 & 71.97 \\
\hline 425 & 8.36 & 16.58 & 23.03 & 0.82 & 1.63 & 70.34 \\
\hline 300 & 5.77 & 11.44 & 11.59 & 0.62 & 1.23 & 69.11 \\
\hline 212 & 4.81 & 9.54 & 2.05 & $\overline{0.47}$ & 0.93 & 68.18 \\
\hline 150 & 0.95 & 1.88 & 0.17 & $\overline{0.58}$ & 1.15 & 67.03 \\
\hline 106 & 0.04 & 0.079 & 0.091 & 4.75 & 9.49 & 57.54 \\
\hline 75 & 0.03 & 0.059 & 0.032 & 8.26 & 16.51 & $\overline{41.03}$ \\
\hline 53 & & & & 16.66 & 33.3 & 7.73 \\
\hline 38 & & & & 3.56 & 7.11 & 0.62 \\
\hline Pan & & & & 0.27 & 0.53 & 0.09 \\
\hline Total & 50.41 & & & 50.02 & & \\
\hline
\end{tabular}

200 g media, 20 minutes, $80 \%$ CS, No magnet

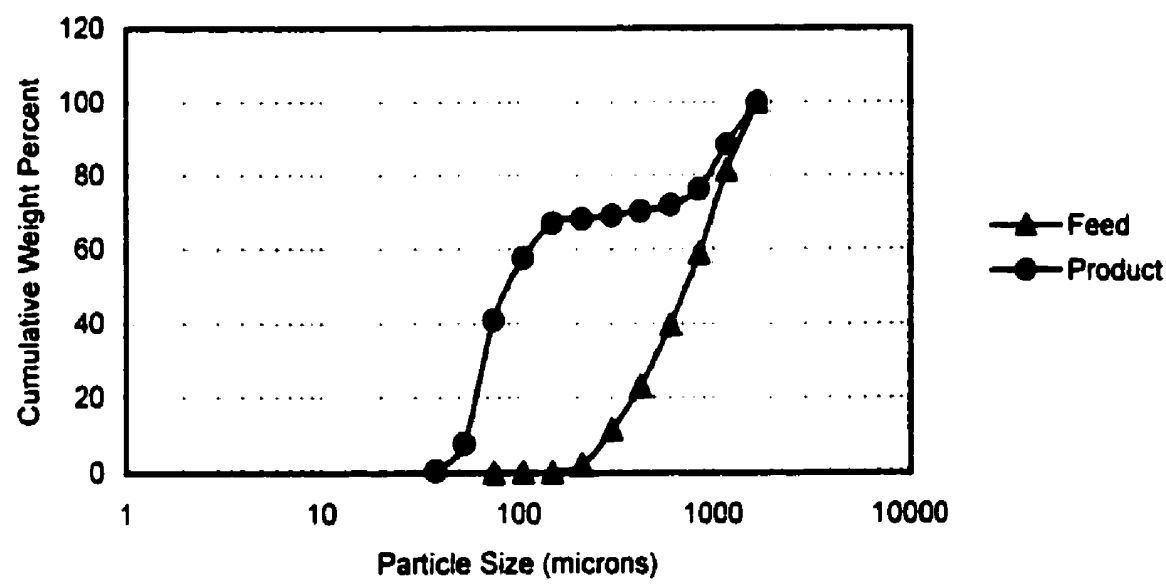




\section{Appendix A3}

Improved Ball Mill

$200 \mathrm{~g}$ media, 20 minutes, $87 \%$ CS, Magnet

\begin{tabular}{|c|c|c|c|c|c|c|}
\hline & Grinding & with ma & gnet Cor & ifiguratio & & \\
\hline Aperture & Before Gri & inding & & After Grin & ding & \\
\hline microns & Weight & Weight\% & Cum.\%F & Weight & Weight \% & Cum.\% F \\
\hline 1700 & 0 & 0 & 100 & 0 & 0 & 100 \\
\hline 1180 & 9.92 & 19.54 & 80.36 & 1.82 & 3.63 & 96.37 \\
\hline 850 & 12.77 & 25.26 & 55.1 & 3.14 & 6.27 & 90.1 \\
\hline 600 & 9.64 & 19.07 & 36.03 & 4.29 & 8.56 & 81.54 \\
\hline$\overline{425}$ & 7.75 & 15.33 & 20.7 & $\overline{5.54}$ & 11.05 & $\overline{70.49}$ \\
\hline 300 & 5.78 & 11.43 & 9.27 & 6.67 & 13.31 & 57.18 \\
\hline 212 & 3.94 & 7.79 & 1.48 & 6.61 & 13.19 & 43.99 \\
\hline 150 & 0.67 & 1.33 & $\overline{0.15}$ & 4.08 & 8.14 & 35.85 \\
\hline 106 & 0.03 & 0.059 & 0.091 & 3.11 & 6.21 & 29.64 \\
\hline 75 & 0.04 & 0.079 & 0.012 & 2.91 & 5.81 & 23.93 \\
\hline 53 & 0.02 & 0.039 & 0 & 11.94 & 23.83 & 0.1 \\
\hline 38 & & & & & & \\
\hline an & & & & & & \\
\hline Total & 50.56 & & & 50.11 & & \\
\hline
\end{tabular}

200 g media,20minutes,87\%CS, Magnet

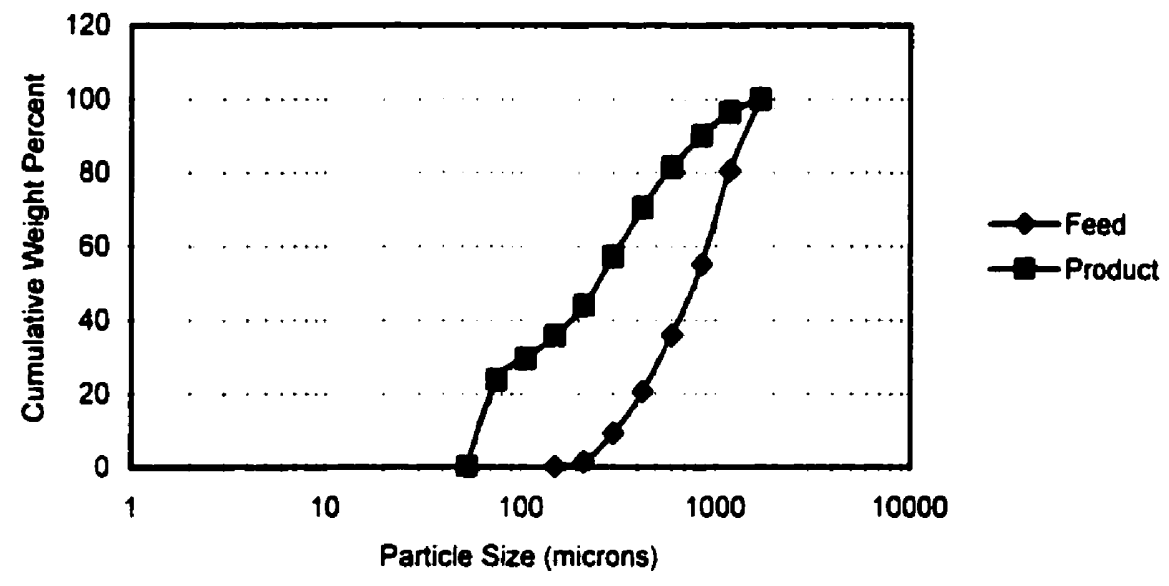




\section{Appendix A3}

\section{Conventional Ball Mill}

200 g media, 20 minutes, 87\%CS, No magnet

\begin{tabular}{|r|r|r|r|r|r|r|}
\hline & \multicolumn{9}{|l|}{ Grinding without magnet } & & & \\
\hline Aperture & \multicolumn{1}{|l|}{ Before Grinding } & & \multicolumn{1}{l|}{ After Grinding } & \\
\hline microns & Weight & Weight\% & Cum. \%F & Weight & Weight\% & Cum.\%F \\
\hline 1700 & 0 & & 100 & 0 & & 100 \\
\hline 1180 & 9.75 & 19.28 & 80.72 & 4.96 & 9.85 & 90.15 \\
\hline 850 & 11.8 & 23.34 & 57.38 & 5.57 & 11.06 & 79.09 \\
\hline 600 & 9.65 & 19.09 & 38.29 & 2.84 & 5.64 & 73.45 \\
\hline 425 & 7.9 & 15.62 & 22.67 & 1.09 & 2.16 & 71.29 \\
\hline 300 & 5.77 & 11.41 & 11.26 & 1.59 & 3.15 & 68.14 \\
\hline 212 & 4.81 & 9.51 & 1.75 & 0.74 & 1.47 & 66.67 \\
\hline 150 & 0.73 & 1.44 & 0.31 & 0.66 & 1.31 & 65.36 \\
\hline 106 & 0.05 & 0.0989 & 0.211 & 1.68 & 3.33 & 62.03 \\
\hline 75 & 0.02 & 0.039 & 0.172 & 9.41 & 18.69 & 43.34 \\
\hline 53 & & & & 16.78 & 33.33 & 10.01 \\
\hline 38 & & & & 4.57 & 9.07 & 0.94 \\
\hline Pan & & & & 0.45 & 0.893 & 0.04 \\
\hline Total & 50.55 & & & 50.34 & & \\
\hline
\end{tabular}

200 g media, 20 minutes,87\%CS, No magnet

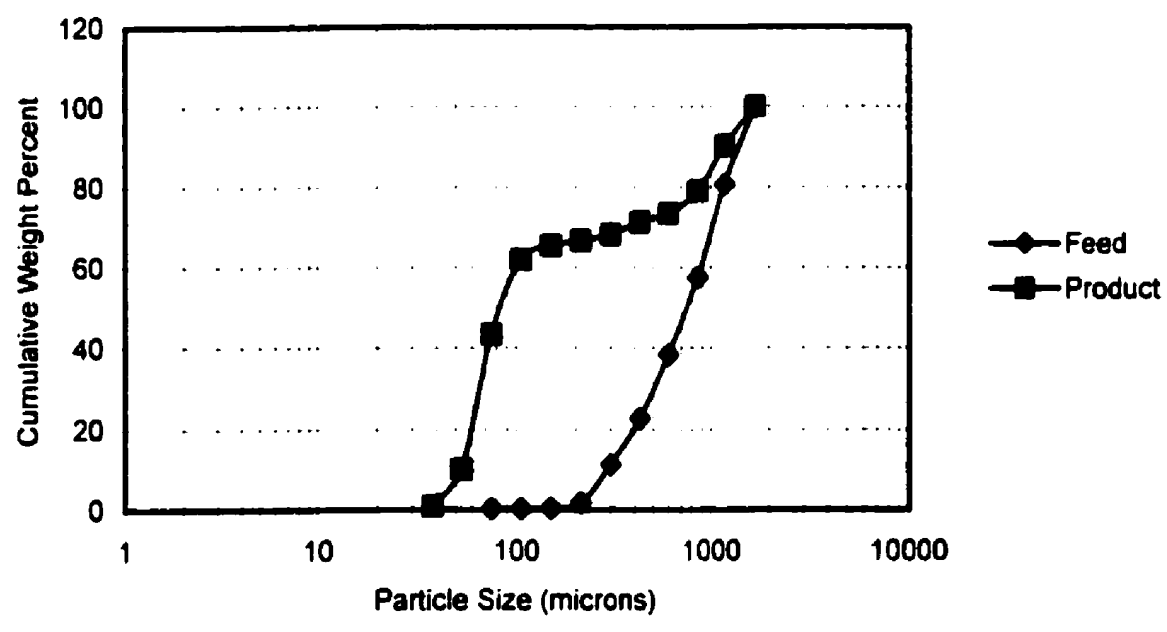




\section{Appendix B1}

Improved Ball Mill

350 media, 20minutes, 70\%CS, Magnot

\begin{tabular}{|r|r|r|r|r|r|r|}
\hline \multicolumn{6}{|l|}{ Grinding with magnet } & \\
\hline Aperture & \multicolumn{1}{l|}{ Before Grinding } & & \multicolumn{2}{l|}{ After Grinding } & \\
\hline microns & Weight & Weight\% & Cum. \%F & Weight & Weight\% & Cum. \%F \\
\hline 1700 & 0 & 0 & 100 & 0 & 0 & 100 \\
\hline 1180 & 10.86 & 21.34 & 78.66 & 4.75 & 9.53 & 90.47 \\
\hline 850 & 12.19 & 23.63 & 55.03 & 4.48 & 8.99 & 81.48 \\
\hline 600 & 9.59 & 18.85 & 36.18 & 2.01 & 4.03 & 77.45 \\
\hline 425 & 7.47 & 14.68 & 21.5 & 0.82 & 1.64 & 75.81 \\
\hline 300 & 5.36 & 10.53 & 10.97 & 0.62 & 1.25 & 74.56 \\
\hline 212 & 4.4 & 8.64 & 2.33 & 0.8 & 1.61 & 72.95 \\
\hline 150 & 0.8 & 1.57 & 0.76 & 0.97 & 1.94 & 71.01 \\
\hline 106 & 0.1 & 0.196 & 0.564 & 2.61 & 5.24 & 65.77 \\
\hline 75 & 0.03 & 0.058 & 0.506 & 16.61 & 33.35 & 32.42 \\
\hline 53 & 0.05 & 0.098 & 0.408 & 13.51 & 27.12 & 5.3 \\
\hline 38 & 0.02 & 0.0393 & 0.368 & 2.1 & 4.22 & 1.08 \\
\hline pan & & & & 0.13 & 0.261 & 0.819 \\
\hline Total & 50.87 & & & 49.81 & & \\
\hline
\end{tabular}

$350 \mathrm{~g}$ media, 20 minutes, $70 \% \mathrm{CS}$, Magnet

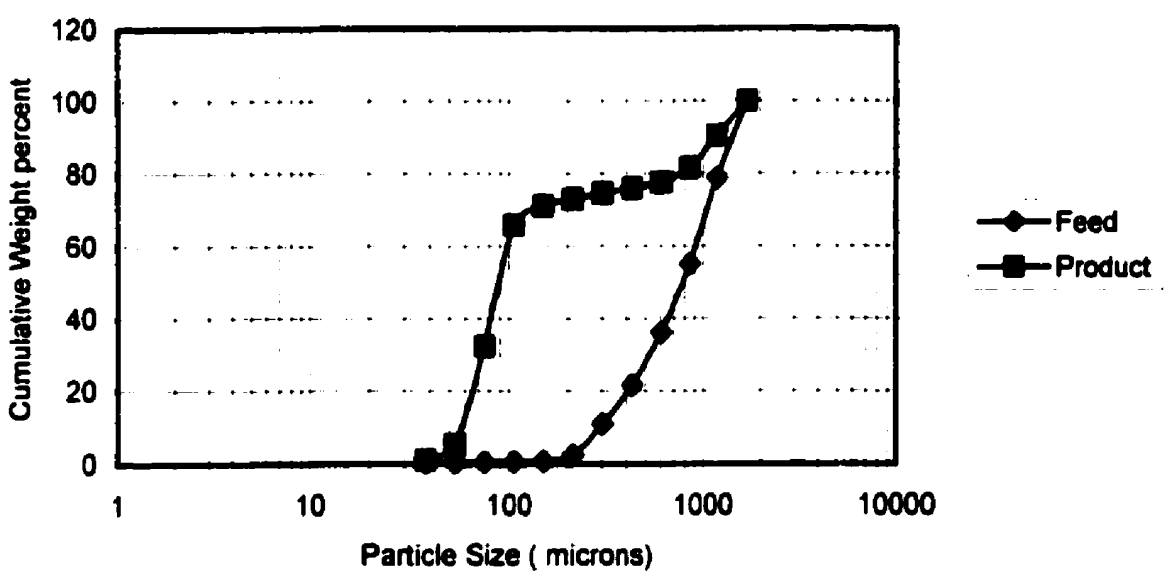




\section{Appendix B1}

\section{Conventional Ball Mill}

20 minutes No magnet, $350 \mathrm{~g}$ media, $70 \% \mathrm{CS}$

\begin{tabular}{|c|c|c|c|c|c|c|}
\hline \multicolumn{7}{|c|}{ Without Magnet Configuration } \\
\hline \multicolumn{3}{|c|}{ Before Grinding } & & \multicolumn{2}{|c|}{ After grinding } & \\
\hline Aperture & Weight & Weight \% & Cum.\% F & Weight & Weight \% & Cum.\% F \\
\hline 1700 & 0 & & 100 & 0 & & 100 \\
\hline 1180 & 8.44 & 16.729 & 83.271 & 4.85 & 9.801 & 90.199 \\
\hline 850 & 12.16 & 24.103 & 59.168 & 7.15 & $\overline{14.45}$ & 75.749 \\
\hline 600 & 9.54 & 18.909 & 40.259 & 3.79 & 7.659 & 68.09 \\
\hline 425 & 8.58 & 17.006 & 23.253 & 1.83 & 3.698 & 64.392 \\
\hline 300 & 5.79 & 11.476 & 11.777 & 1.77 & 3.577 & 60.815 \\
\hline 212 & 4.91 & 9.732 & 2.045 & 3.39 & 6.851 & 53.964 \\
\hline 150 & 0.92 & 1.823 & 0.222 & 1.64 & 3.314 & 50.65 \\
\hline$\overline{106}$ & 0.09 & 0.178 & 0.044 & 4.24 & 8.569 & 42.081 \\
\hline 75 & 0.02 & 0.0396 & 0.004 & 17.38 & 35.125 & 6.956 \\
\hline 53 & & & & 3.27 & 6.608 & 0.348 \\
\hline 38 & & & & 0.17 & $0 . \overline{343}$ & 0.005 \\
\hline Pan & & & & & & \\
\hline Total & 50.45 & & & 49.48 & & \\
\hline
\end{tabular}

350 g media, 20 minutes, 70\%CS, No magnot

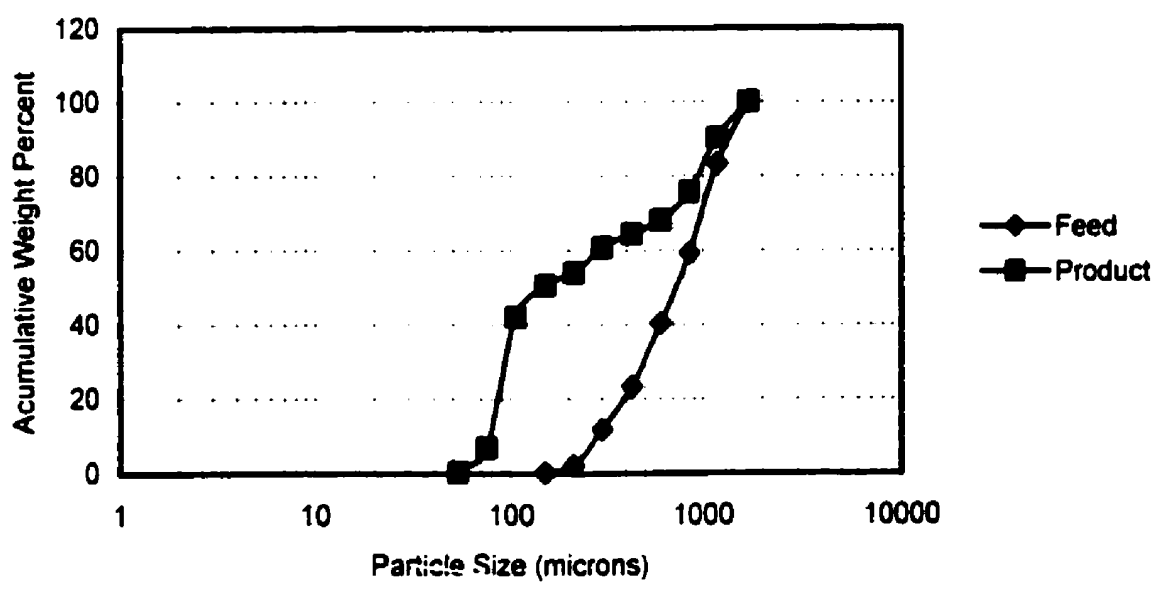




\section{Appendix B2}

Improved Ball Mill

350 g media, 20 minutes, $80 \%$ CS, Magnet

\begin{tabular}{|c|c|c|c|c|c|c|}
\hline \multicolumn{7}{|c|}{ With Magnet Configuration } \\
\hline & \multicolumn{2}{|c|}{ Before Grinding } & & \multicolumn{2}{|c|}{ After grinding } & \\
\hline Aperture & Weight & Weight \% & Cum.\% F & Weight & Weight \% & Cum.\% F \\
\hline 1700 & 0 & & 100 & 0 & & 100 \\
\hline 1180 & 6.71 & 13.266 & 86.734 & 2.7 & 5.451 & 94.549 \\
\hline 850 & 10.39 & 20.541 & 66.193 & 3.55 & 7.167 & 87.382 \\
\hline 600 & 9.86 & 19.493 & 46.7 & 2.67 & 5.391 & 81.991 \\
\hline$\overline{425}$ & 8.7 & 17.201 & $\overline{29.499}$ & 2.15 & 4.341 & 77.65 \\
\hline$\overline{300}$ & $\overline{6.73}$ & 13.305 & $\overline{16.194}$ & 2.98 & 6.016 & 71.634 \\
\hline$\overline{212}$ & 6.79 & 13.242 & 3 & 6.62 & 13.365 & 58.269 \\
\hline 150 & 1.19 & 2.352 & 0.6 & 1.98 & 3.997 & 54.272 \\
\hline 106 & 0.18 & 0.355 & 0.245 & 2.89 & 5.834 & 48.438 \\
\hline 75 & 0.03 & 0.059 & 0.186 & 19.28 & 38.925 & 9.513 \\
\hline 53 & & & & 4.51 & 9.105 & 0.403 \\
\hline 38 & & & & 0.2 & 0.403 & \\
\hline \multicolumn{7}{|l|}{ Pan } \\
\hline Total & 50.58 & & & 49.53 & & \\
\hline
\end{tabular}

350 g media, 20 minutes, $80 \%$ CS, Magnet

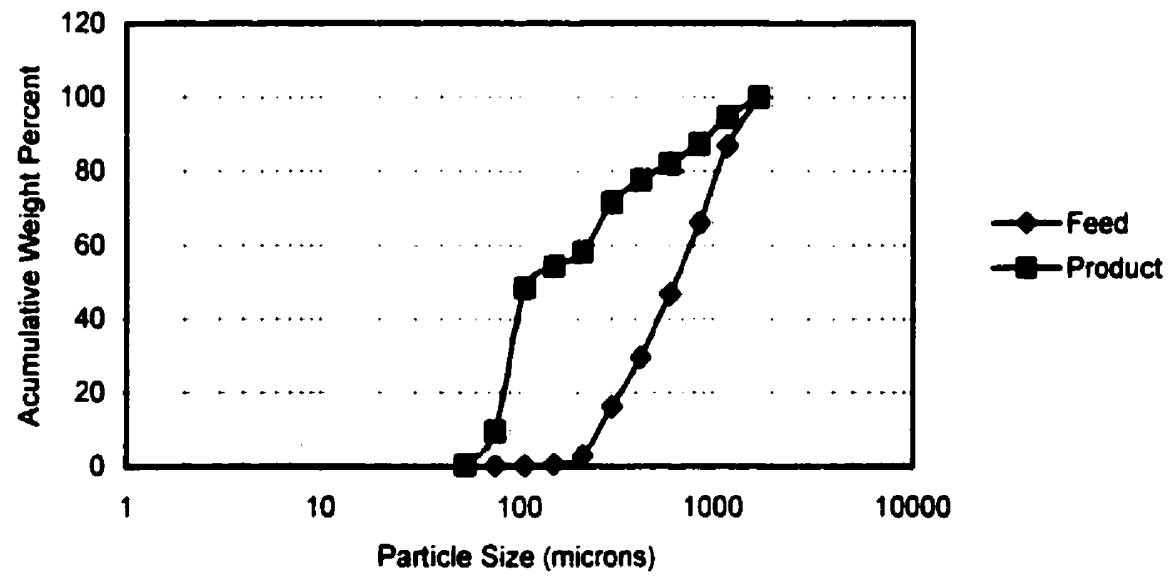




\section{Appendix B2}

\section{Conventional Ball MIII}

$350 g$ media, 20 minutes, $80 \%$ CS, No magne

\begin{tabular}{|r|r|r|r|r|r|r|}
\hline \multicolumn{7}{|c|}{ Without Magnet Configuration } \\
\hline \multicolumn{3}{|c|}{ Before Grinding } & \multicolumn{3}{|c|}{ After grinding } & \\
\hline Aperture & Weight & Weight \% & Cum.\% F & Weight & Weight \% & Cum.\% F \\
\hline 1700 & 0 & & 100 & 0 & & 100 \\
\hline 1180 & 9.13 & 18.079 & 81.921 & 4.41 & 8.869 & 91.131 \\
\hline 850 & 12.18 & 24.118 & 57.803 & 5.34 & 10.74 & 80.391 \\
\hline 600 & 9.45 & 18.712 & 39.091 & 3.72 & 6.878 & 73.513 \\
\hline 425 & 7.69 & 15.227 & 23.864 & 3.29 & 6.617 & 66.896 \\
\hline 300 & 5.74 & 11.366 & 12.498 & 2.42 & 4.867 & 62.029 \\
\hline 212 & 5.92 & 11.722 & 0.776 & 2.51 & 5.048 & 56.981 \\
\hline 150 & 0.39 & 0.772 & 0.004 & 2.44 & 4.907 & 52.074 \\
\hline 106 & 0 & & & 3.75 & 7.542 & 44.532 \\
\hline 75 & 0 & & & 18.35 & 36.906 & 7.626 \\
\hline 53 & 0 & & & 3.2 & 6.436 & 1.19 \\
\hline Pan & 0 & & & 0.29 & 0.583 & 0.607 \\
\hline Total & 50.5 & & & & & \\
\hline
\end{tabular}

350 g media, 20minutes, $80 \%$ CS, No magnet

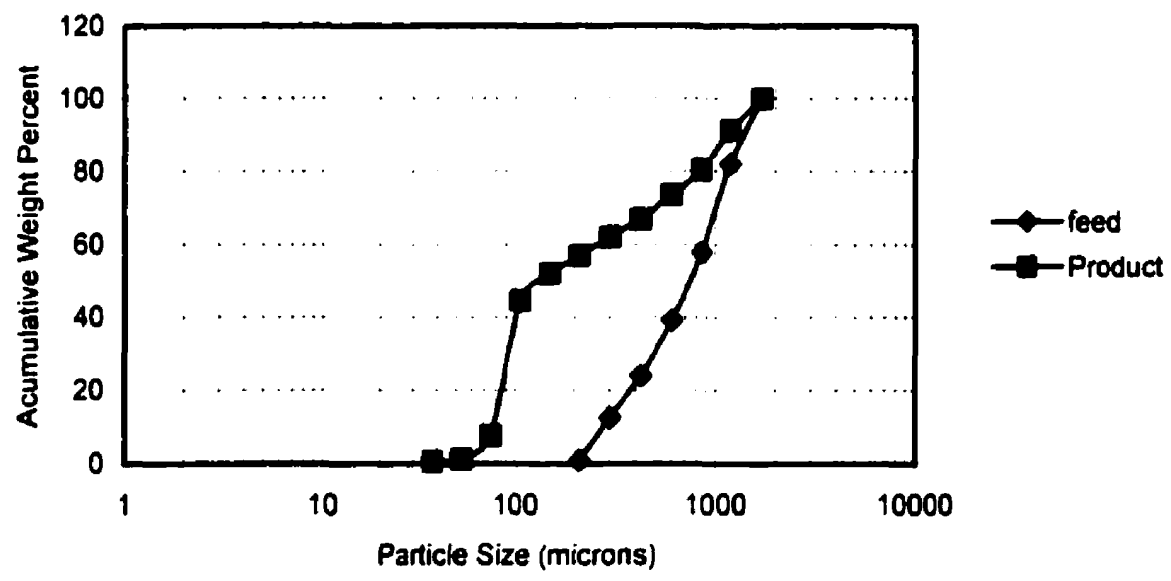




\section{Appendix B3}

Conventional Ball Mill

350 g media, 20minutes, $87 \%$ CS, No magnet

\begin{tabular}{|r|r|r|r|r|r|r|}
\hline \multicolumn{7}{|l|}{ Grinding without magnet } \\
\hline Aperture & \multicolumn{1}{|l|}{ Bofore Grinding } & & \multicolumn{1}{|l|}{ After Grinding } & \\
\hline microns & Weight & Weight\% & Cum. \% & Weight & Weight\% & Cum.\%F \\
\hline 1700 & 0 & 0 & 100 & 0 & 0 & 100 \\
\hline 1180 & 10.86 & 21.34 & 78.66 & 4.75 & 9.53 & 90.47 \\
\hline 850 & 12.19 & 23.63 & 55.03 & 4.48 & 8.99 & 81.48 \\
\hline 600 & 9.59 & 18.85 & 36.18 & 2.01 & 4.03 & 77.45 \\
\hline 425 & 7.47 & 14.68 & 21.5 & 0.82 & 1.64 & 75.81 \\
\hline 300 & 5.36 & 10.53 & 10.97 & 0.62 & 1.25 & 74.56 \\
\hline 212 & 4.4 & 8.64 & 2.33 & 0.8 & 1.61 & 72.95 \\
\hline 150 & 0.8 & 1.57 & 0.76 & 0.97 & 1.94 & 71.01 \\
\hline 106 & 0.1 & 0.196 & 0.564 & 2.61 & 5.24 & 65.77 \\
\hline 75 & 0.03 & 0.058 & 0.506 & 16.61 & 33.35 & 32.42 \\
\hline 53 & 0.05 & 0.098 & 0.408 & 13.51 & 27.12 & 5.3 \\
\hline 38 & 0.02 & 0.0393 & 0.368 & 2.1 & 4.22 & 1.08 \\
\hline pan & & & & 0.13 & 0.261 & 0.819 \\
\hline Total & 50.87 & & & 49.81 & & \\
\hline
\end{tabular}

350 g media, 20 minutes, $87 \%$ CS, No magnet

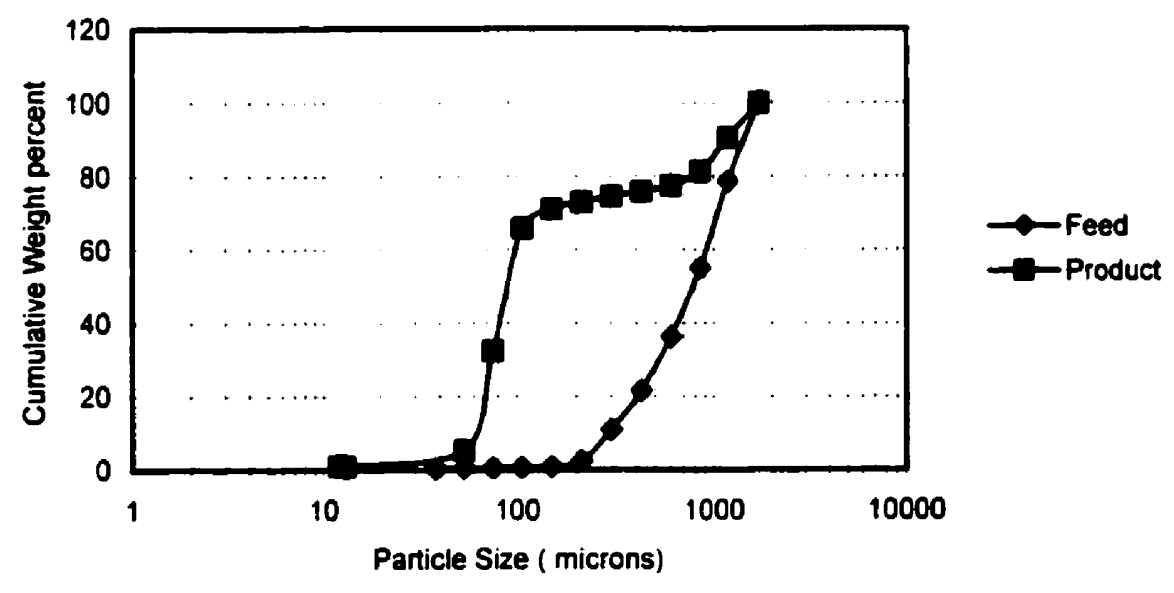


Appendix B3

Improved Ball Mill

350 g media, 20 minutes, 87\%CS, Magnet

\begin{tabular}{|c|c|c|c|c|c|c|}
\hline \multirow{3}{*}{$\begin{array}{l}\text { Aperture } \\
\text { microns }\end{array}$} & \multicolumn{5}{|c|}{ Grinding with magnet Configuration } & \multirow[b]{3}{*}{ Cum.\% F } \\
\hline & Before Gn & inding & & After Grinc & ding & \\
\hline & Weight & Weight\% & Cum.\%F & Weight & Weight \% & \\
\hline 1700 & 0 & 0 & 100 & 0 & 0 & 100 \\
\hline 1180 & 8.99 & 17.68 & 82.32 & 1.96 & 3.91 & 96.09 \\
\hline 850 & 12.06 & $\overline{23.72}$ & 58.6 & 2.32 & 4.62 & 91.47 \\
\hline 600 & 9.95 & 19.57 & 39.03 & 1.82 & 3.62 & 87.85 \\
\hline 425 & 7.83 & 15.4 & 23.63 & 4.86 & 9.69 & 78.16 \\
\hline 300 & 6.31 & $\overline{12.41}$ & 11.22 & 5.69 & 11.34 & 66.82 \\
\hline 212 & $\overline{4.84}$ & 9.52 & 1.67 & 8.05 & 16.05 & 50.77 \\
\hline 150 & $\overline{0.84}$ & 1.65 & 0.019 & 5.24 & 10.45 & 40.32 \\
\hline 106 & 0.01 & 0.019 & 0 & 4.14 & 8.25 & 32.07 \\
\hline 75 & & & & 3.58 & 7.13 & 24.94 \\
\hline 53 & & & & 9.23 & 18.41 & 6.53 \\
\hline 38 & & & & 3.18 & 6.34 & 0.19 \\
\hline Pan & & & & 0.08 & 0.159 & 0.03 \\
\hline Total & 50.83 & & & 50.15 & & \\
\hline
\end{tabular}

350 g media, 20 minutes, $87 \%$ CS, Magnet

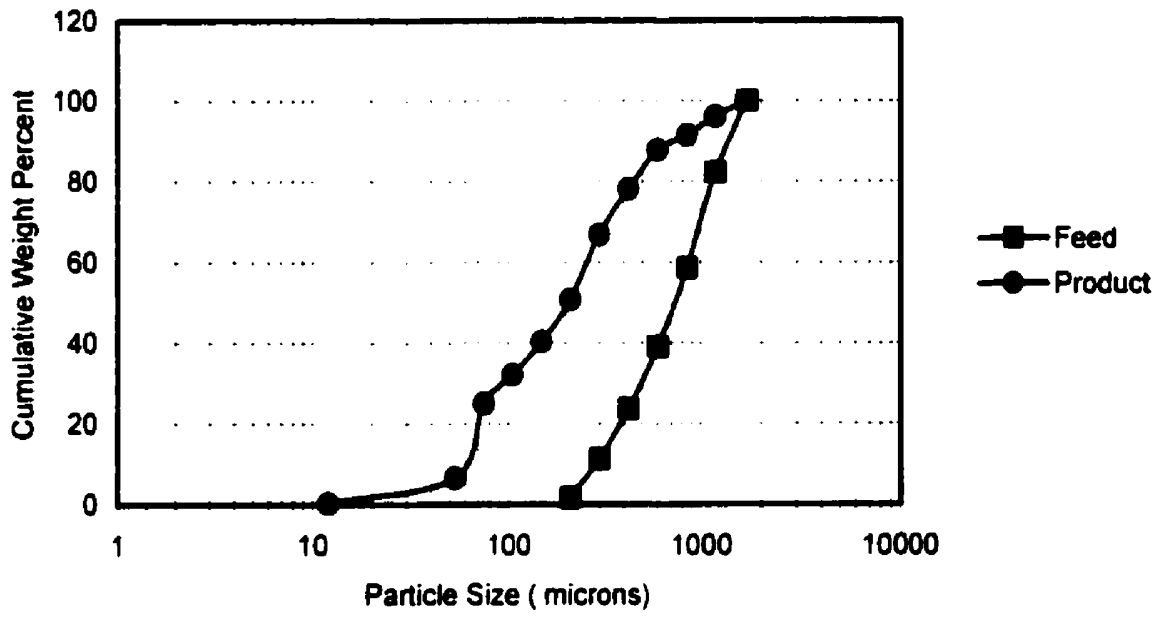


Appendix C1

Improved Ball Mill

$750 g$ media, 20 minutes, 70\%CS, Magnet

\begin{tabular}{|c|c|c|c|c|c|c|}
\hline \multicolumn{7}{|c|}{ With Magnet Configuration } \\
\hline & \multicolumn{2}{|c|}{ Before Grinding } & & \multicolumn{2}{|c|}{ After grinding } & \\
\hline Aperture & Weight & Weight \% & Cum.\% F & Weight & Weight \% & Cum.\% F \\
\hline 1700 & 0 & & 100 & $\overline{0}$ & & 100 \\
\hline 1180 & 9.18 & 18.121 & 81.88 & 3.25 & 6.55 & 93.45 \\
\hline 850 & 11.85 & 23.391 & 58.489 & 3.85 & 7.76 & 85.69 \\
\hline 600 & 10.05 & 19.838 & 38.651 & 2.56 & 5.16 & 80.53 \\
\hline 425 & 7.74 & 15.278 & 23.373 & 2.38 & 4.8 & $\overline{75.73}$ \\
\hline 300 & 5.59 & 11.034 & 12.339 & 4.19 & 8.45 & 67.68 \\
\hline 212 & 5.77 & 11.389 & 0.95 & 5.06 & 10.21 & 57.47 \\
\hline 150 & 0.48 & 0.947 & 0.003 & $\overline{4.39}$ & 8.85 & 48.62 \\
\hline 106 & 0 & & & 5.85 & 11.8 & 36.82 \\
\hline 75 & 0 & & & 13.95 & 28.15 & 8.67 \\
\hline 53 & 0 & & & 3.66 & 7.38 & 1.33 \\
\hline 38 & 0 & & & 0.61 & 1.23 & 0.1 \\
\hline Pan & 0 & & & & & \\
\hline Total & 50.66 & & & 49.55 & & \\
\hline
\end{tabular}

760 gedia,20minutes,70\%CS, magnet

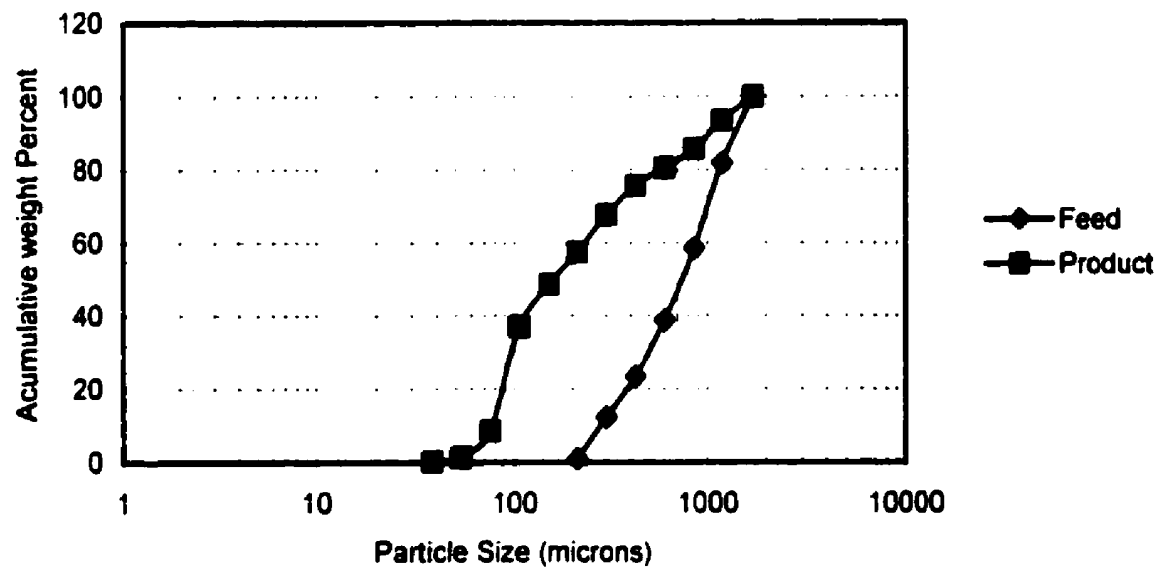




\section{Appendix C1}

Conventional Ball Mill

$750 g$ media , 20 minutes, 70\%CS, No magnet

\begin{tabular}{|c|c|c|c|c|c|c|}
\hline & Grinding & without & magnet & Configur & ation & \\
\hline Aperture & Bofore Gn & inding & & After Grin & ding & \\
\hline microns & Weight & Weight\% & Cum.\%F & Weight & Weight \% & Cum.\% F \\
\hline 1700 & . & 0 & 100 & 然 & 0 & 100 \\
\hline 1180 & 9.24 & 18.24 & 81.76 & 4.44 & 8.87 & 91.13 \\
\hline 850 & 11.65 & 23 & 58.76 & 5.75 & 11.49 & 79.64 \\
\hline 600 & 9.6 & 18.95 & 39.81 & 8.21 & 16.41 & 63.23 \\
\hline 425 & 7.92 & 15.64 & 24.17 & 7.58 & 15.15 & 48.08 \\
\hline 300 & 6.54 & 12.91 & 11.26 & 7.41 & $\overline{14.81}$ & 33.27 \\
\hline 212 & 4.79 & 9.46 & 1.8 & 5.88 & 11.75 & 21.52 \\
\hline 150 & 0.84 & 1.66 & 0.12 & 2.96 & 5.91 & 15.61 \\
\hline 106 & 0.06 & 0.12 & 0 & 2.61 & 5.21 & 10.4 \\
\hline 75 & & & & 2.12 & 4.23 & 6.17 \\
\hline 53 & & & & 2.47 & 4.93 & 1.24 \\
\hline 38 & & & & 0.58 & 1.15 & 0.09 \\
\hline Pan & & & & & & \\
\hline Total & 50.64 & & & $\overline{50.05}$ & & \\
\hline
\end{tabular}

750 g media, 20 minutes, $70 \%$ CS, No magnet

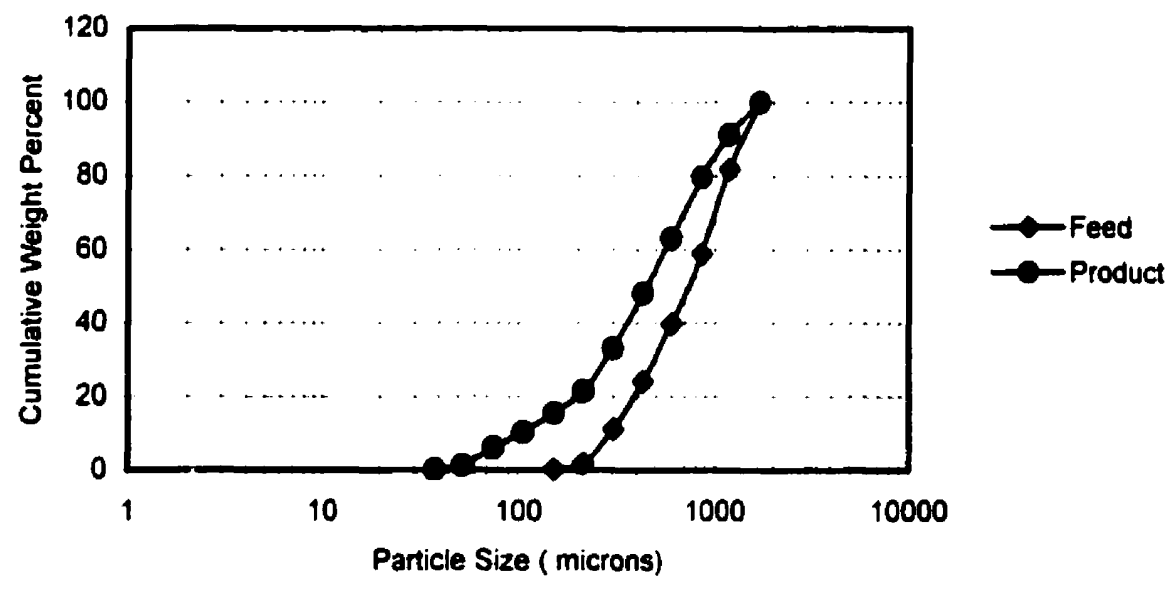




\section{Appendix C2}

\section{Conventional Ball mill}

750 g medla, 20 minutes, $80 \% C S$, No magnet

\begin{tabular}{|c|c|c|c|c|c|c|}
\hline \multicolumn{7}{|c|}{ Without Magnet Configuration } \\
\hline \multicolumn{3}{|c|}{ Before Grinding } & \multirow[b]{2}{*}{ Cum.\%F } & \multicolumn{2}{|c|}{ After grinding } & \multirow[b]{2}{*}{ Cum.\% F } \\
\hline Aperture & Weight & Weight \% & & Weight & Weight \% & \\
\hline 1700 & $\overline{0}$ & & 100 & & & 100 \\
\hline 1180 & 6.07 & 11.99 & 88.01 & 4.74 & 9.5 & 90.5 \\
\hline 850 & 9.52 & 18.81 & 69.2 & 4.69 & 9.4 & 81.1 \\
\hline 600 & 9.57 & $\overline{18.91}$ & 50.29 & 3.49 & $\overline{6.99}$ & $\overline{74.11}$ \\
\hline 425 & 9.11 & 18 & 32.29 & 2.42 & 4.85 & 69.26 \\
\hline 300 & 7.02 & 13.87 & 18.42 & 2.38 & 4.77 & 64.49 \\
\hline 212 & 7.3 & 14.42 & 4 & 4.09 & 8.19 & 56.3 \\
\hline 150 & 1.63 & 3.22 & 0.78 & 4.51 & 9.04 & 47.26 \\
\hline 106 & 0.24 & 0.474 & 0.306 & 9.07 & 18.18 & 29.08 \\
\hline 75 & 0.08 & 0.158 & 0.148 & 10.69 & 21.43 & 7.65 \\
\hline 53 & 0.06 & 0.118 & 0.03 & 3.8 & 7.61 & 0.04 \\
\hline 38 & 0 & & & & & \\
\hline Pan & 0 & & & & & \\
\hline Total & 50.6 & & & 49.88 & & \\
\hline
\end{tabular}

750 g media, 20 minutes, $80 \%$ CS, No magnet

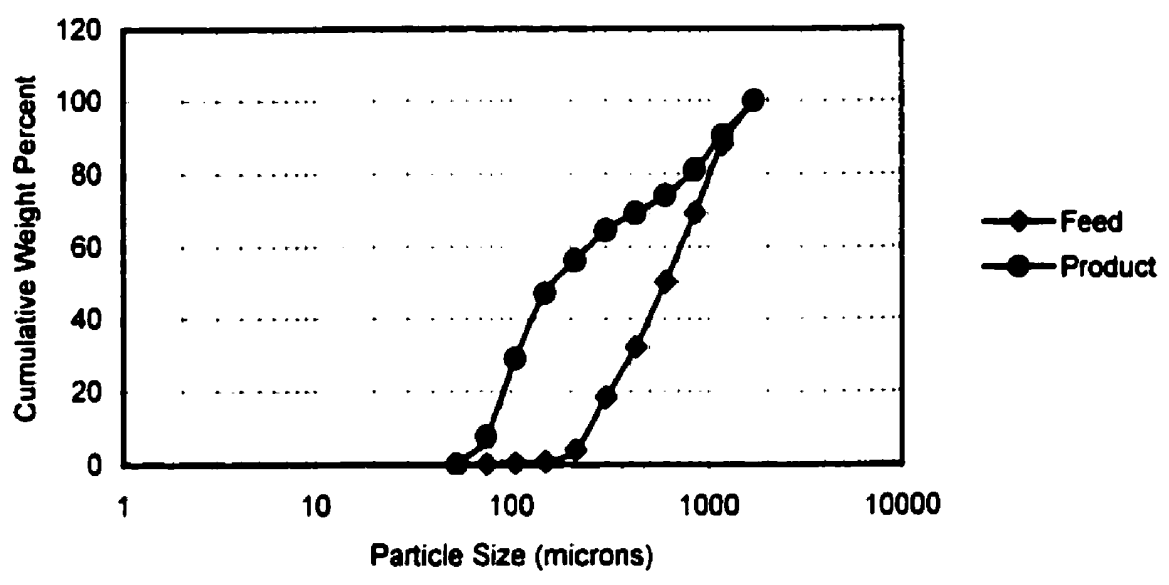




\section{Appendix C2}

Improved Ball mill

$750 \mathrm{~g}$ media, 20 minutes, 80\%CS, Magnet

\begin{tabular}{|r|r|r|r|r|r|r|}
\hline \multicolumn{6}{|l|}{ Grinding with magnot Configuration } & \\
\hline Aperture & \multicolumn{1}{|l|}{ Bofore Grinding } & \multicolumn{3}{l|}{ After Grinding } & \\
\hline microns & Weight & Weight\% & Cum. \%F & Weight & Weight \% & Cum.\% F \\
\hline 1700 & 0 & 0 & 100 & 0 & 0 & 100 \\
\hline 1180 & 10.67 & 21.25 & 78.75 & 1.88 & 3.76 & 96.24 \\
\hline 850 & 12.13 & 24.15 & 54.6 & 1.68 & 3.36 & 92.88 \\
\hline 600 & 9.19 & 18.3 & 36.3 & 2.78 & 5.57 & 87.31 \\
\hline 425 & 7.54 & 15.01 & 21.29 & 4.01 & 8.04 & 79.27 \\
\hline 300 & 5.18 & 10.31 & 10.98 & 5.75 & 11.52 & 67.75 \\
\hline 212 & 4.44 & 8.84 & 2.14 & 6.45 & 12.93 & 54.82 \\
\hline 150 & 0.94 & 1.87 & 0.27 & 4.48 & 9.98 & 44.84 \\
\hline 106 & 0.09 & 0.179 & 0.091 & 3.94 & 7.9 & 36.93 \\
\hline 75 & 0.03 & 0.059 & & 7.29 & 14.61 & 22.32 \\
\hline 53 & & & & 9.68 & 19.41 & 3.86 \\
\hline 38 & & & & 1.82 & 3.64 & 0.23 \\
\hline Pan & & & & 0.11 & 0.221 & \\
\hline Total & 50.21 & & & 49.87 & & \\
\hline
\end{tabular}

750 g media, 20 minutes, $80 \% \%$ CS, Magnet

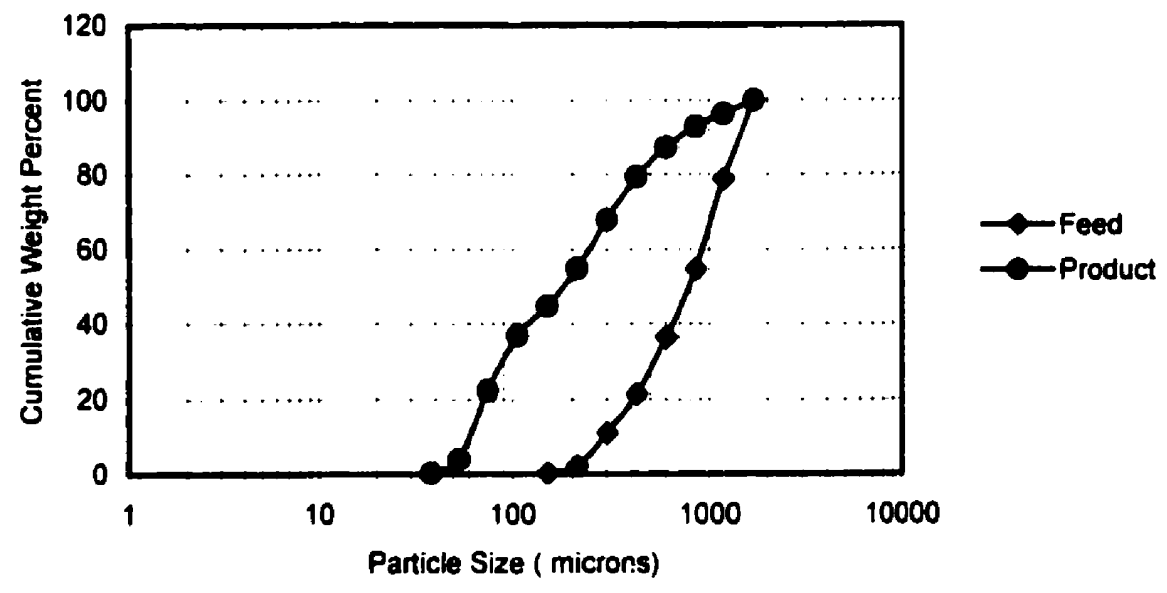




\section{Appendix C3}

\section{Improved Ball mill}

$750 g$ media, 20 minutes, 87\%CS, magnet

\begin{tabular}{|c|c|c|c|c|c|c|}
\hline & \multicolumn{5}{|c|}{ Grinding with magnot Configuration } & \\
\hline Aperture & Before Gn & nding & & After Grin & ding & \\
\hline microns & Weight & Weight\% & Cum.\%F & Weight & Weight \% & Cum.\% F \\
\hline 1700 & 0 & 0 & 100 & $\overline{0}$ & 0 & 100 \\
\hline 1180 & 10.67 & 21.25 & 78.75 & 1.78 & 3.57 & 96.43 \\
\hline 850 & 12.13 & 24.15 & 54.6 & 1.48 & 2.97 & 93.46 \\
\hline 600 & 9.19 & 18.3 & 36.3 & 2.38 & 4.77 & 88.69 \\
\hline 425 & $\overline{7.54}$ & 15.01 & 21.29 & 4.01 & 8.04 & 80.65 \\
\hline 300 & 5.18 & 10.31 & 10.98 & 5.75 & 11.52 & 69.13 \\
\hline 212 & 4.44 & 8.84 & 2.14 & 6.45 & 12.93 & 56.2 \\
\hline 150 & 0.94 & 1.87 & 0.27 & 4.48 & 9.98 & 46.22 \\
\hline 106 & 0.09 & 0.179 & 0.091 & 3.94 & 7.91 & 38.31 \\
\hline 75 & 0.03 & 0.059 & & 7.69 & 15.42 & 22.89 \\
\hline 53 & & & & 9.68 & 19.41 & 4.48 \\
\hline 38 & & & & 2.12 & 4.25 & 0.23 \\
\hline Pan & & & & 0.11 & 0.221 & 0.03 \\
\hline Total & 50.21 & & & 49.87 & & \\
\hline
\end{tabular}

750 g media, 20 minutes, $87 \% \%$ CS, Magnet

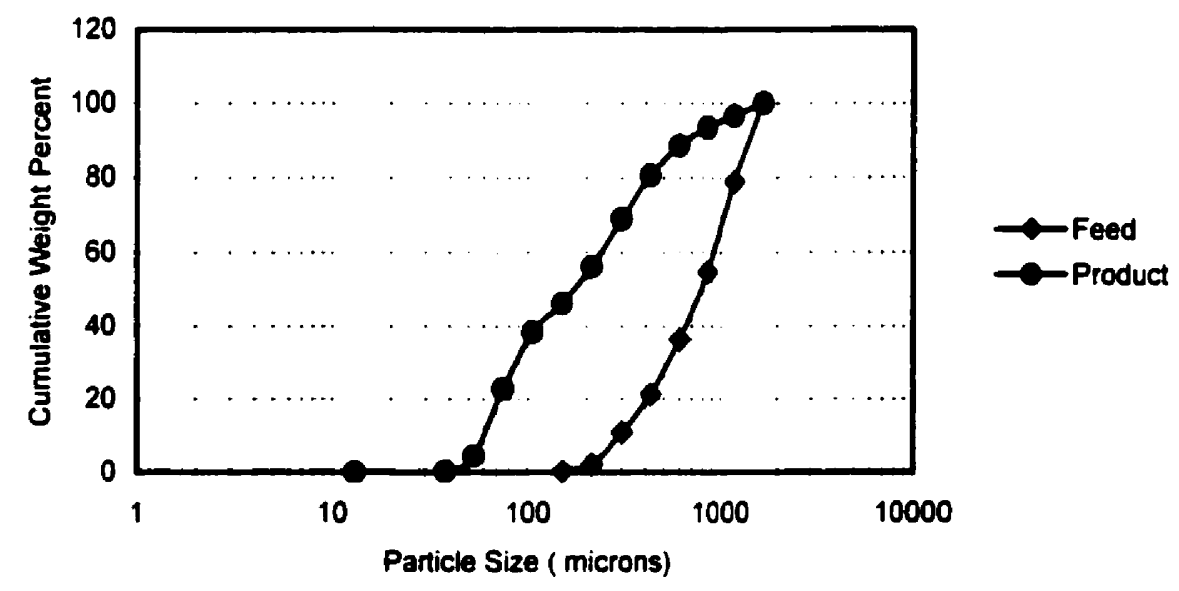


Appendix C3

Conventional Ball mill

$750 \mathrm{~g}$ medla, 20 minutes, 87\%CS, No magnet

\begin{tabular}{|c|c|c|c|c|c|c|}
\hline \multicolumn{7}{|c|}{ Without Magnet Configuration } \\
\hline \multicolumn{3}{|c|}{ Before Grinding } & & \multicolumn{2}{|c|}{ After grinding } & \\
\hline Aperture & Weight & Weight \% & Cum.\% F & Weight & Weight \% & Cum.\% F \\
\hline 1700 & 0 & & 100 & 0 & & 100 \\
\hline 1180 & 6.14 & 12.26 & 87.74 & 3.18 & 6.41 & 93.59 \\
\hline 850 & 10.15 & 20.27 & 67.47 & 4.91 & 9.91 & 83.68 \\
\hline 600 & 9.43 & 18.83 & 48.64 & 4.46 & 8.99 & 74.69 \\
\hline 425 & 9.14 & 18.25 & 30.39 & 5.01 & 10.11 & 64.58 \\
\hline 300 & 6.71 & $\mathbf{1 3 . 4}$ & 16.99 & $\overline{4.34}$ & 8.75 & 55.83 \\
\hline 212 & 6.52 & $\overline{13.24}$ & 3.75 & $\overline{4.44}$ & 8.95 & 46.88 \\
\hline 150 & 1.31 & 2.61 & 1.14 & 3.57 & 7.2 & 39.68 \\
\hline 106 & 0.14 & 0.279 & 1.039 & 3.62 & 7.3 & 32.38 \\
\hline 75 & 0.51 & 1.02 & 0.019 & 7.58 & 15.29 & 17.09 \\
\hline 53 & 0.01 & 0.019 & & 7.3 & 14.72 & 2.37 \\
\hline 38 & 0 & & & 1.11 & 2.24 & 0.13 \\
\hline Pan & 0 & & & 0.04 & 0.081 & 0.05 \\
\hline Total & 50.06 & & & 49.55 & & \\
\hline
\end{tabular}

750 g media, 20 minutes, 87\%CS, No magnet

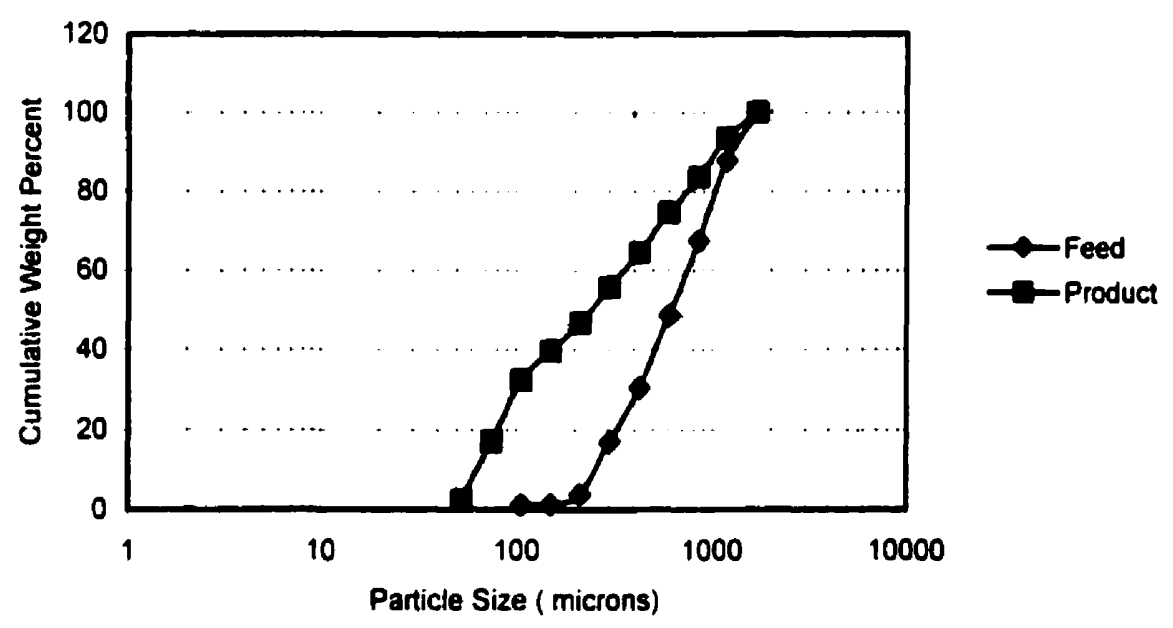




\section{Addendix D1}

Effect of Sample Weight
Conventional Ball Mill

350 g media, 20 minutes, 87\%CS, No magnet, 50g Sample

\begin{tabular}{|c|c|c|c|c|c|c|}
\hline \multirow{3}{*}{$\begin{array}{l}\text { Aperture } \\
\text { microns }\end{array}$} & \multicolumn{5}{|c|}{ Grinding without magnet Configuration } & \multirow[b]{3}{*}{ Cum.\% F } \\
\hline & \multicolumn{2}{|c|}{ Bofore Grinding } & & \multicolumn{2}{|c|}{ After Grinding } & \\
\hline & Weight & Weight\% & Cum.\%F & Weight & Weight \% & \\
\hline 1700 & 0 & 0 & 100 & 0 & 0 & 100 \\
\hline 1180 & 9.12 & 17.9811 & 82.0189 & 7.27 & 14.54 & 85.46 \\
\hline 850 & 11.23 & 22.1411 & 59.8778 & 8.33 & 16.66 & 68.8 \\
\hline 600 & 8.03 & 15.832 & 44.0458 & 4.34 & 8.68 & 60.12 \\
\hline 425 & 7.31 & 14.4124 & 29.6334 & 3.42 & 6.84 & 53.28 \\
\hline 300 & 5.98 & 11.7902 & 17.8432 & 3.32 & 6.64 & 46.64 \\
\hline 212 & 7.33 & 14.4518 & 3.3914 & 2.97 & 5.94 & 40.7 \\
\hline 150 & 1.45 & 2.8588 & 0.5326 & 2.38 & 4.76 & 35.94 \\
\hline$\overline{106}$ & 0.25 & 0.4929 & 0.0397 & 15.03 & 30.07 & 5.87 \\
\hline 75 & 0.02 & 0.0394 & 0.0003 & 2.58 & 5.16 & 0.71 \\
\hline 53 & & & & 0.3 & 0.6 & 0.11 \\
\hline 38 & & & & 0.04 & 0.08 & 0.03 \\
\hline Pan & & & & & & \\
\hline Total & 50.72 & & & 49.98 & & \\
\hline
\end{tabular}

350 g media, 20 minutes, 87\%CS, No magnet, 50g Sample

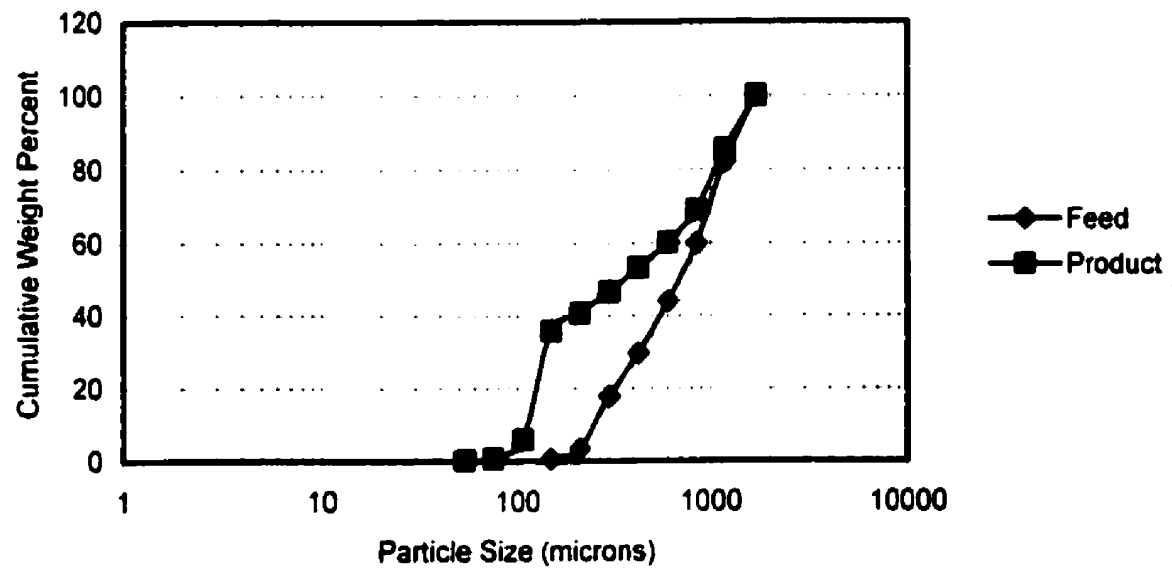


Addendix D1

Effect of Sample Weight

Improved Ball Mill

350 media, 20 minutes, $87 \%$ CS, Magnet, $50 \mathrm{~g}$ Sample

\begin{tabular}{|c|c|c|c|c|c|c|}
\hline & Grinding & with ma & Ignet Con & Ifiguratio & & \\
\hline Aperture & Bofore Gr & inding & & After Grin & ding & \\
\hline microns & Weight & Weight\% & Cum.\%F & Weight & Weight \% & Cum.\% F \\
\hline 1700 & 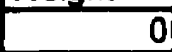 & 0 & 100 & 0 & 0 & 100 \\
\hline 1180 & 8.44 & 16.7161 & 83.2839 & 2.91 & 5.87 & 94.13 \\
\hline 850 & 11.43 & 22.6381 & 60.6458 & 2.84 & 5.73 & 88.4 \\
\hline 600 & 7.9 & 15.6466 & 44.9992 & 2.16 & 4.36 & 84.04 \\
\hline 425 & 7.07 & 14.0027 & 30.9965 & 2.95 & 5.95 & 78.09 \\
\hline 300 & 6.09 & 12.0617 & 18.9348 & 1.97 & 3.97 & 74.3 \\
\hline 212 & 7.81 & 15.4684 & 3.4664 & 1.84 & 3.71 & 70.59 \\
\hline 150 & 1.49 & 2.9511 & 0.5153 & 1.97 & 3.97 & 66.62 \\
\hline 106 & 0.26 & 0.5149 & 0.0004 & 30.66 & 61.88 & 4.74 \\
\hline 75 & & & & 1.99 & 4.01 & 0.73 \\
\hline 53 & & & & 0.24 & 0.48 & 0.25 \\
\hline 38 & & & & & & \\
\hline Pan & & & & & & \\
\hline Total & 50.49 & & & 49.54 & & \\
\hline
\end{tabular}

350 g media, 20 minutes, 87\%CS, Magnet, 50g Sample

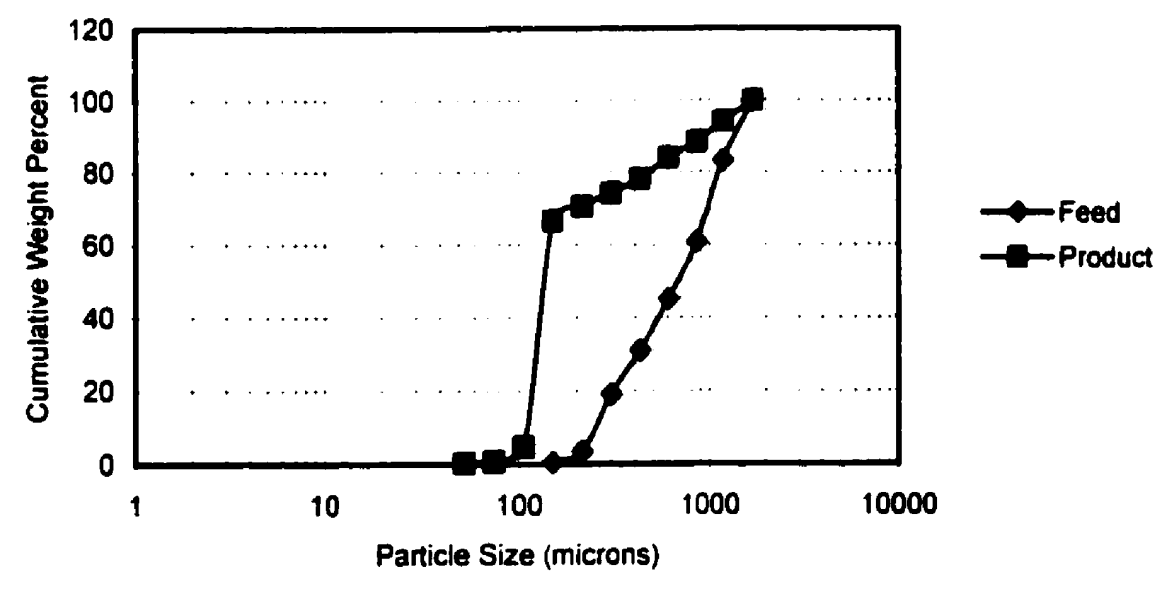




\section{Appendix D2}

\section{Calculation of the constant $C$ at different conditions of operations}

1. $350 \mathrm{~g}$ Modia, 87\% of Critical Speed , C = 2.4562

\begin{tabular}{|c|c|c|c|c|c|c|c|}
\hline Time & 80\%Pass. & $80 \%$ Pass. & 80\%Pass. & constant & Energy & Average & Energy \\
\hline minutes & Feed & No Mag. & Mag. & C & Mag. & C & No Mag. \\
\hline 5 & 1200 & 1050 & 930 & 1.9688 & 0.5237 & 2.4562 & 1.2864 \\
\hline 10 & 1200 & 980 & 720 & 2.7309 & 1.1213 & 2.4562 & 2.7543 \\
\hline 15 & 1200 & 880 & 560 & 2.7653 & 1.7875 & 2.4562 & 4.3905 \\
\hline 20 & 1200 & 790 & 470 & 2.5719 & 2.3041 & 2.4562 & 5.6591 \\
\hline 25 & 1200 & 670 & 380 & 2.2969 & 2.9945 & 2.4562 & 7.3552 \\
\hline 30 & 1200 & 610 & 310 & 2.4032 & 3.7284 & 2.4562 & 9.1577 \\
\hline & & & Total & 14.737 & & & \\
\hline & & & Average & 2.4562 & & & $40.70 \%$ \\
\hline
\end{tabular}

2- $\quad 350 \mathrm{~g}$ Media, $80 \%$ of Critical Speed, $\mathrm{C}=\quad 2.6696$

\begin{tabular}{|c|c|c|c|c|c|c|c|}
\hline Time & $80 \%$ Pass. & $80 \%$ Pass. & $80 \%$ Pass. & constant & Energy & Average & Energy \\
\hline minutes & Feed & No Mag. & Mag. & C & Mag. & C & No Mag. \\
\hline 5 & 1200 & 1100 & 980 & 2.3971 & 0.4106 & 2.6696 & 1.0962 \\
\hline 10 & 1200 & 1020 & 810 & 2.5656 & 0.8368 & 2.6696 & 2.2341 \\
\hline 15 & 1200 & 970 & 640 & 3.2902 & 1.4231 & 2.6696 & 3.7993 \\
\hline 20 & 1200 & 840 & 510 & 2.7351 & 2.0576 & 2.6696 & 5.4929 \\
\hline 25 & 1200 & 720 & 400 & 2.5157 & 2.8211 & 2.6696 & 7.5313 \\
\hline 30 & 1200 & 680 & 360 & 2.5143 & 3.1821 & 2.6696 & 8.4952 \\
\hline & & & $\begin{array}{l}\text { Total } \\
\text { Average }\end{array}$ & $\begin{array}{l}16.018 \\
2.6696\end{array}$ & & & $37.50 \%$ \\
\hline
\end{tabular}




\section{Appendix D2}

3- $750 g$ Media, $87 \%$ of Critical Speed, C =

\begin{tabular}{|c|c|c|c|c|c|c|c|}
\hline Time & 80\%Pass. & $80 \%$ Pass. & $80 \%$ Pass. & constant & Energy & Average & Energy \\
\hline minutes & Feed & No Mag. & Mag. & C & Mag. & C & No Mag. \\
\hline 5 & 1200 & 1040 & 910 & 2.0001 & 0.5716 & 2.4039 & 1.3741 \\
\hline 10 & 1200 & 960 & 700 & 2.6207 & 1.1919 & 2.4039 & 2.8653 \\
\hline 15 & 1200 & 860 & 520 & 2.8642 & 2.0004 & 2.4039 & 4.8089 \\
\hline 20 & 1200 & 740 & $\overline{420}$ & 2.5247 & 2.6602 & 2.4039 & 6.3949 \\
\hline 25 & 1200 & 620 & 340 & 2.2461 & 3.3861 & 2.4039 & 8.1401 \\
\hline 30 & 1200 & 550 & 290 & 2.1677 & 3.9855 & 2.4039 & 9.5807 \\
\hline & & & Total & 14.4235 & & & \\
\hline & & & Average & 2.4039 & & & $41.59 \%$ \\
\hline
\end{tabular}

4- $750 \mathrm{~g}$ Media, $80 \%$ of Critical Speed, C =

\begin{tabular}{|r|r|r|r|r|r|r|r|}
\hline Time & $80 \%$ Pass. & $80 \%$ Pass. & $80 \%$ Pass. & Constant & Energy & Average & Energy \\
\hline minutes & Feed & No Mag. & Mag. & C & Mag. & C & No Mag. \\
\hline 5 & 1200 & 1060 & 920 & 2.2206 & 0.5474 & 2.5325 & 1.3865 \\
\hline 10 & 1200 & 1000 & 790 & 2.4359 & 0.8958 & 2.5325 & 2.2687 \\
\hline 15 & 1200 & 910 & 610 & 2.7141 & 1.5513 & 2.5325 & 3.9288 \\
\hline 20 & 1200 & 810 & 440 & 2.9999 & 2.5104 & 2.5325 & 6.3578 \\
\hline 25 & 1200 & 680 & 370 & 2.4387 & 3.0864 & 2.5325 & 7.8165 \\
\hline 30 & 1200 & 630 & 330 & 2.3858 & 3.4951 & 2.5325 & 8.8512 \\
\hline & & & 15.195 & & & \\
\hline
\end{tabular}




\section{Appendix D2}

\section{Particle Size/ Energy}

$750 \mathrm{~g}$ Media, $87 \%$ of Critical Speed, C = $\quad 2.4039$

\begin{tabular}{|r|r|r|r|r|}
\hline Time & \multicolumn{1}{|l|}{$80 \%$ Pass. } & $80 \%$ Pass. & Energy & Energy \\
\hline minutes & \multicolumn{1}{|l}{ Feed } & Mag. & Mag. & No Mag. \\
\hline 5 & 1200 & 910 & 0.5716 & 1.3741 \\
\hline 10 & 1200 & 700 & 1.1919 & 2.8653 \\
\hline 15 & 1200 & 520 & 2.0004 & 4.8089 \\
\hline 20 & 1200 & 420 & 2.6602 & 6.3949 \\
\hline 25 & 1200 & 340 & 3.3861 & 8.1401 \\
\hline 30 & 1200 & 290 & 3.9855 & 9.5807 \\
\hline
\end{tabular}

Particle Size/Energy, $\mathbf{C}=\mathbf{2 . 4 0 3 9}$

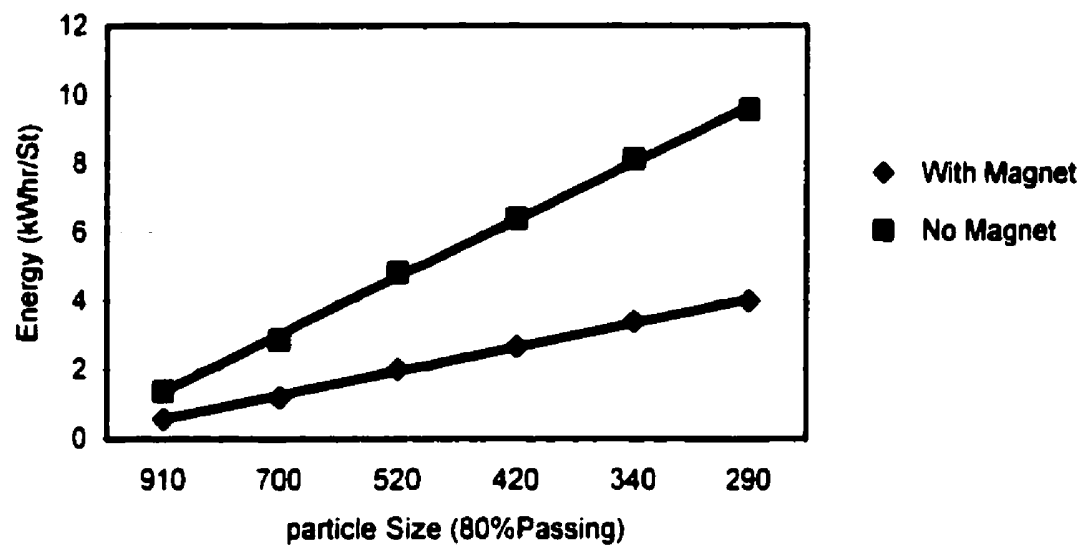




\section{Appendix $\mathrm{D2}$}

\section{Particle Size/ Energy}

$750 \mathrm{~g}$ Media, $80 \%$ of Critical Spe $\quad 2.5325$

\begin{tabular}{|r|r|r|r|r|}
\hline Time & $80 \%$ Pass. & $80 \%$ Pass. & Energy & Energy \\
\hline minutes & \multicolumn{1}{|l|}{ Feed } & Mag. & Mag. & No Mag. \\
\hline 5 & 1200 & 920 & 0.5474 & 1.3865 \\
\hline 10 & 1200 & 790 & 0.8958 & 2.2687 \\
\hline 15 & 1200 & 610 & 1.5513 & 3.9288 \\
\hline 20 & 1200 & 440 & 2.5104 & 6.3578 \\
\hline 25 & 1200 & 370 & 3.0864 & 7.8165 \\
\hline 30 & 1200 & 330 & 3.4951 & 8.8512 \\
\hline
\end{tabular}

Particle Size/ Energy, $C=\mathbf{2 . 5 3}$

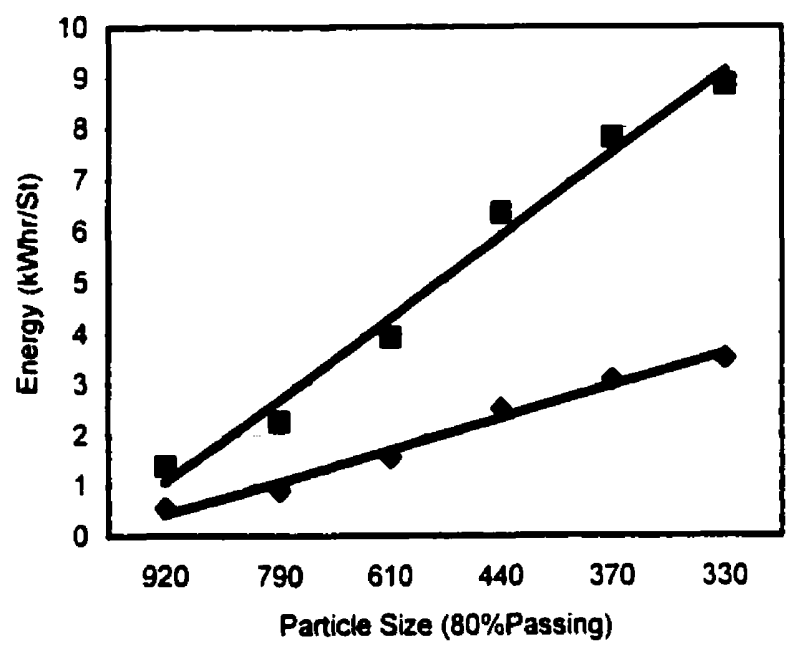

- With Magnet

- No Magnet 
Appendix D2

Particle Size/ Energy

$350 \mathrm{~g}$ Media, $80 \%$ of Critical Speed, C = 2.6696

\begin{tabular}{|r|r|r|r|r|}
\hline Time & \multicolumn{1}{|l|}{$80 \%$ Pass. } & $80 \%$ Pass. & Energy & Energy \\
\hline minutes & Feed & Mag. & Mag. & No Mag. \\
\hline 5 & 1200 & 980 & 0.4106 & 1.0962 \\
\hline 10 & 1200 & 810 & 0.8368 & 2.2341 \\
\hline 15 & 1200 & 640 & 1.4231 & 3.7993 \\
\hline 20 & 1200 & 510 & 2.0576 & 5.4929 \\
\hline 25 & 1200 & 400 & 2.8211 & 7.5313 \\
\hline 30 & 1200 & 360 & 3.1821 & 8.4952 \\
\hline
\end{tabular}

Particle Size/ Energy, $\mathrm{C}=\mathbf{2 . 6 6}$

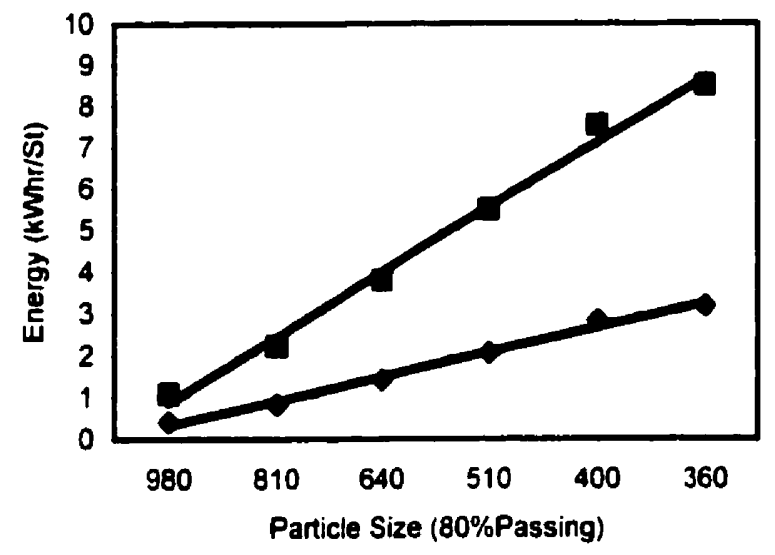

- With Magnet

- No Magnet 


\section{Appendix D2}

Particle Sizel Energy

$350 \mathrm{~g}$ Media, 87\% of Critical Speed , C $=2.4562$

\begin{tabular}{|r|r|l|l|r|}
\hline Time & \multicolumn{1}{|l|}{$80 \%$ Pass. } & $80 \%$ Pass. & Energy & Energy \\
\hline minutes & Feed & Mag. & Mag. & No Mag. \\
\hline 5 & 1200 & 930 & 0.5237 & 1.2864 \\
\hline 10 & 1200 & 720 & 1.1213 & 2.7543 \\
\hline 15 & 1200 & 560 & 1.7875 & 4.3905 \\
\hline 20 & 1200 & 470 & 2.3041 & 5.6591 \\
\hline 25 & 1200 & 380 & 2.9945 & 7.3552 \\
\hline 30 & 1200 & 310 & 3.7284 & 9.1577 \\
\hline
\end{tabular}

Energy/Particle Size, C $=\mathbf{2 . 4 5 6 2}$

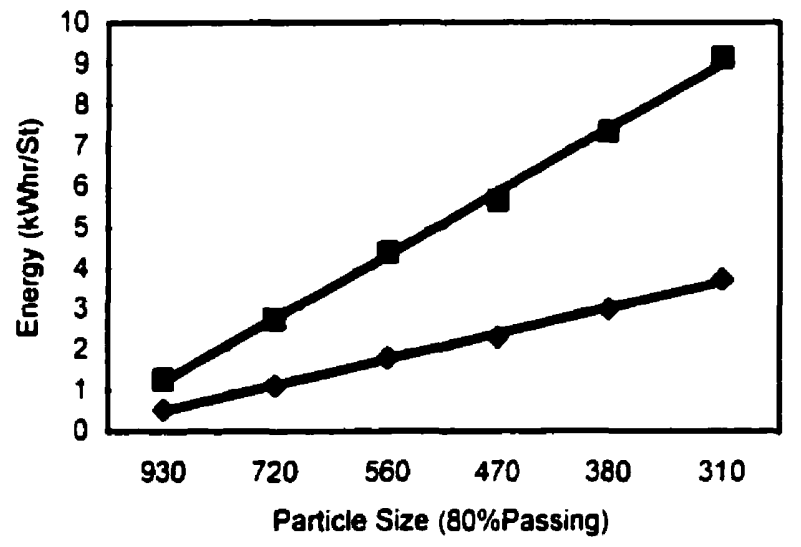

- With Magnet

- No Magnet 


\section{Appendix E1}

Testing the Optimum Condition of Operation

Effect of the Sample Weight

Media: 350grams

Speed 87\%

Time: 20 minutes

\begin{tabular}{|r|r|r|}
\hline Sample & $80 \%$ Pass. & $80 \%$ Pass. \\
\hline Grams & No Mag & Mag. \\
\hline 25 & 970 & 280 \\
\hline 35 & 915 & 370 \\
\hline 50 & 1030 & $\mathbf{4 7 0}$ \\
\hline 75 & 1050 & 520 \\
\hline 100 & 1080 & 980 \\
\hline
\end{tabular}

Effect of Woight of Sample

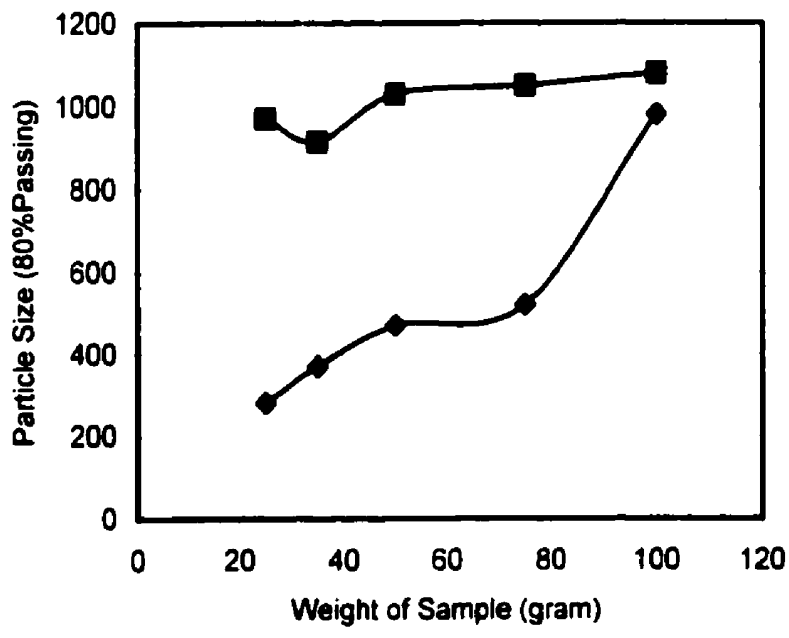

-With Magnet

- without mag. 


\section{Appendix E1}

Testing the Optimum Condition of Operation Effect of Media

Speed: $\mathbf{8 7 \%}$

Sample: 50grams

Time: 20 minutes

\begin{tabular}{|r|r|r|}
\hline Media & $80 \%$ Pass. & $80 \%$ ass. \\
\hline grams & No Mag. & Mag. \\
\hline 175 & 990 & 980 \\
\hline 350 & 1030 & 470 \\
\hline 500 & 995 & 370 \\
\hline 750 & 980 & 300 \\
\hline
\end{tabular}

Effect of Media Woight

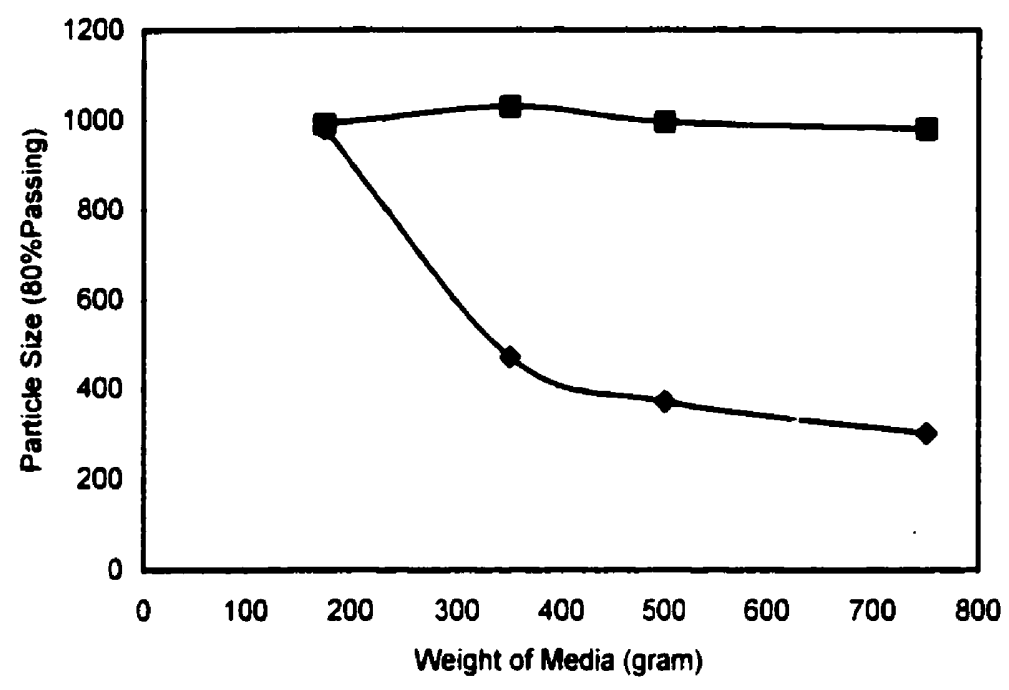

with Mag.

-without

Mag. 


\section{Appendix E1}

\section{Testing the Optimum Condition of Operation \\ Effect of Rotational Speed}

\section{Sample: 50grams}

Modia: 350grams

Time: 20 minutes

\begin{tabular}{|r|r|r|}
\hline R. Speed & 805Pass. & 80\%Pass. \\
\hline & No Mag. & Mag. \\
\hline 70 & 930 & 800 \\
\hline 80 & 840 & 510 \\
\hline 87 & 790 & 470 \\
\hline 95 & 860 & 720 \\
\hline 98 & 920 & 830 \\
\hline
\end{tabular}

Effect of Rotational Speed

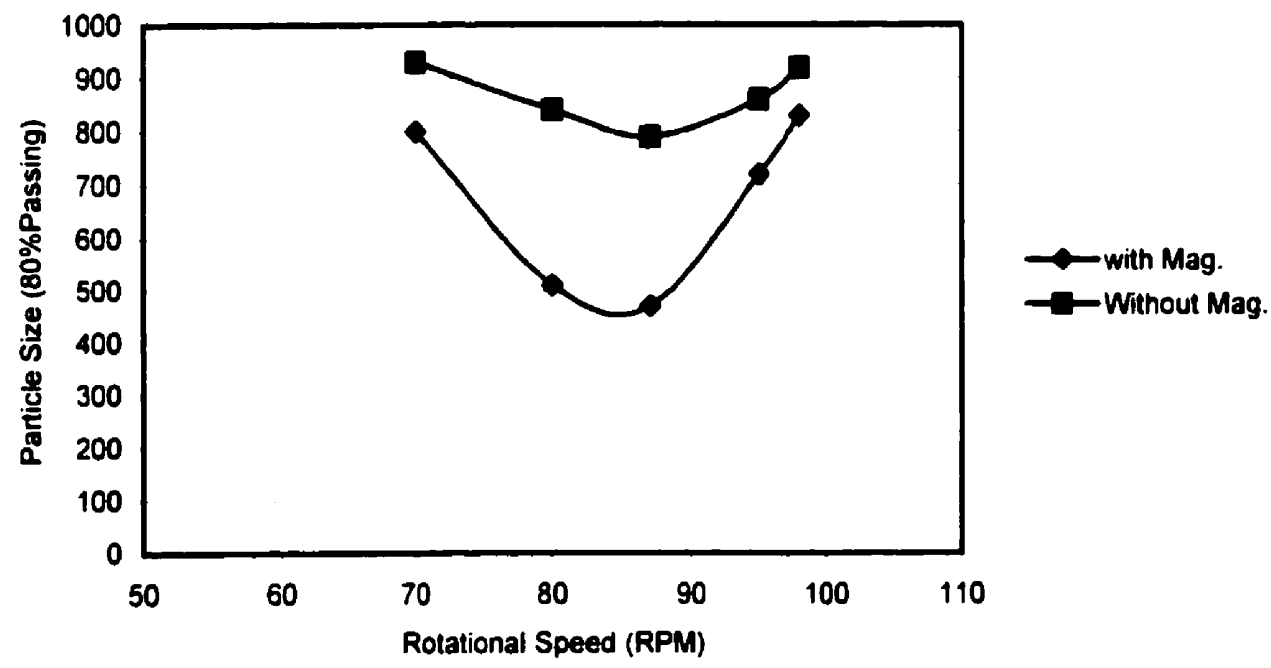




\section{Appendix E2}

\section{Improved Ball Mill \\ Effect of Modia Loading \\ Rotational Spee 80\%}

\begin{tabular}{|c|c|c|c|c|}
\hline & & $200 \mathrm{~g}$ & $350 \mathrm{~g}$ & $750 \mathrm{~g}$ \\
\hline Time & $80 \%$ Pass. & $80 \%$ Pass. & $80 \%$ Pass. & $80 \%$ Pass. \\
\hline minutes & Feed & Mag. & Mag. & Mag. \\
\hline 5 & 1200 & 1080 & 980 & 920 \\
\hline 10 & 1200 & 930 & 810 & 790 \\
\hline 15 & 1200 & 720 & 640 & 610 \\
\hline 20 & 1200 & 600 & 510 & 440 \\
\hline 25 & 1200 & 520 & 400 & 370 \\
\hline 30 & $\overline{1200}$ & 430 & 360 & 330 \\
\hline
\end{tabular}

Effect of media,80\%CS, Improved Ball Mill

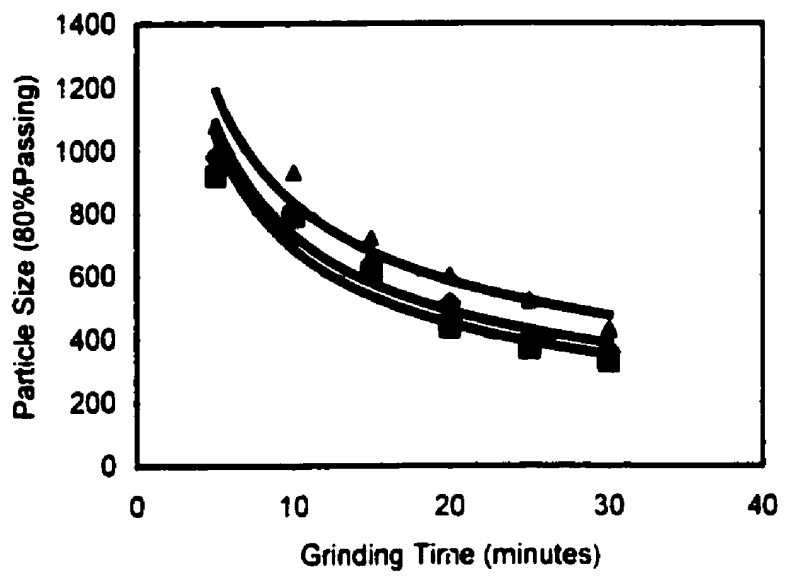

- $350 \mathrm{~g}$

d 7509

- 2009 
Appendix E2

Improved ball Mill

Effoct of Modia Loading

Rotational speed 87\%

\begin{tabular}{|r|r|l|l|l|}
\hline & & \multicolumn{1}{|l|}{$200 \mathrm{~g}$} & $350 \mathrm{~g}$ & $750 \mathrm{~g}$ \\
\hline Time & \multicolumn{1}{|l|}{$80 \%$ Pass. } & $80 \%$ Pass. & $80 \%$ Pass. & $80 \%$ Pass. \\
\hline minutes & \multicolumn{1}{|c|}{ Feed } & Mag. & Mag. & Mag. \\
\hline 5 & 1200 & 1000 & 930 & 910 \\
\hline 10 & 1200 & 820 & 720 & 700 \\
\hline 15 & 1200 & 730 & 560 & 520 \\
\hline 20 & 1200 & 640 & 470 & 420 \\
\hline 25 & 1200 & 570 & 380 & 340 \\
\hline 30 & 1200 & 460 & 310 & 290 \\
\hline
\end{tabular}

Effect of Media, $87 \%$ CS, Improved Ball Mill

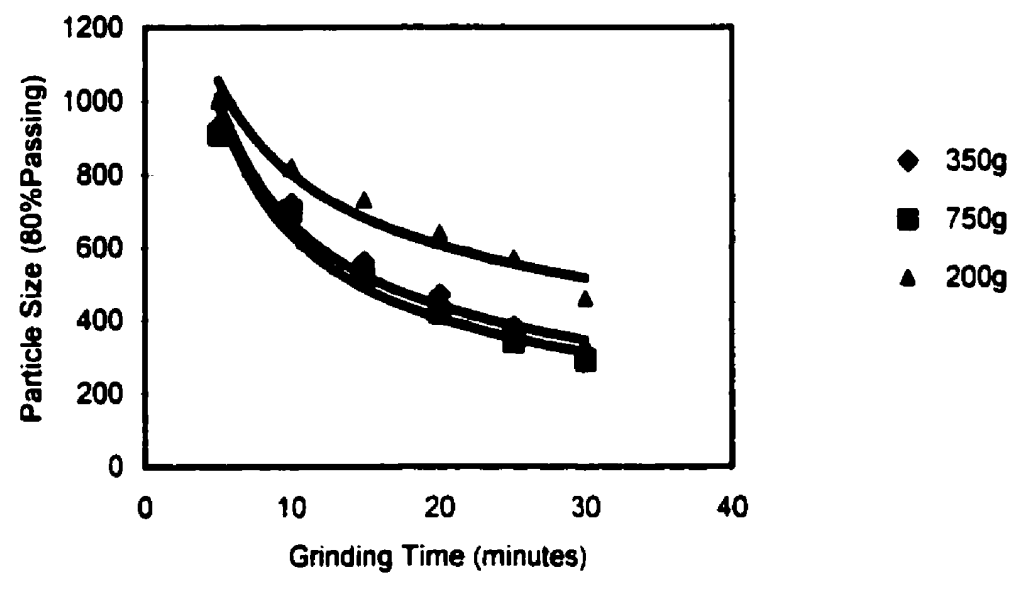




\section{Appendix E2}

\section{Conventional Ball Mill}

Effoct of Modia Loading

Rotational Spee 87\%

\begin{tabular}{|r|r|r|l|l|}
\hline & & $200 \mathrm{~g}$ & $350 \mathrm{~g}$ & $750 \mathrm{~g}$ \\
\hline Time & $80 \%$ Pass. & $80 \%$ Pass. & $80 \%$ Pass. & $80 \%$ Pass. \\
\hline minutes & \multicolumn{1}{|l|}{ Feed } & No Mag. & No Mag. & No Mag. \\
\hline 5 & 1200 & 990 & 930 & 910 \\
\hline 10 & 1200 & 840 & 720 & 700 \\
\hline 15 & 1200 & 670 & 560 & 520 \\
\hline 20 & 1200 & 580 & 470 & 420 \\
\hline 25 & 1200 & 460 & 380 & 340 \\
\hline 30 & 1200 & 410 & 310 & 290 \\
\hline
\end{tabular}

Effect of Media, 87\%CS,Conventional Ball Mill

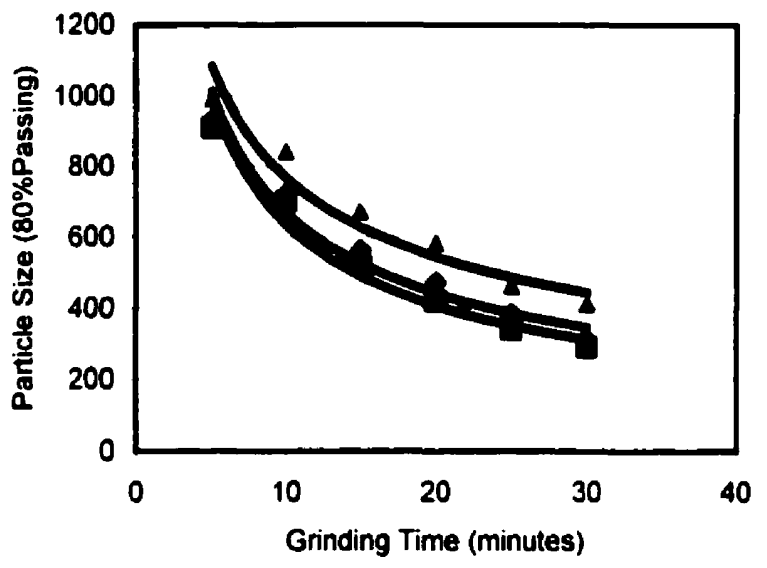

- 3509

- $750 \mathrm{~g}$

- $200 \mathrm{~g}$ 


\section{Appendix E2}

\section{Conventional Ball Mill}

Effoct of Modia Loading

Rotational Speed 80\%

\begin{tabular}{|l|r|r|r|r|}
\hline & & $200 \mathrm{~g}$ & $350 \mathrm{~g}$ & $750 \mathrm{~g}$ \\
\hline Time & $80 \%$ Pass. & $80 \%$ Pass. & $80 \%$ Pass. & $80 \%$ Pass. \\
\hline minutes & Feed & No Mag. & No Mag. & No Mag. \\
\hline 5 & 1200 & 1170 & 1100 & 1060 \\
\hline 10 & 1200 & 1090 & 1020 & 1000 \\
\hline 15 & 1200 & 1020 & 970 & 910 \\
\hline 20 & 1200 & 970 & 840 & 810 \\
\hline 25 & 1200 & 860 & 720 & 680 \\
\hline 30 & 1200 & 760 & 680 & 630 \\
\hline
\end{tabular}

Effect of Media, $80 \%$ CS, Conventional Ball Mill

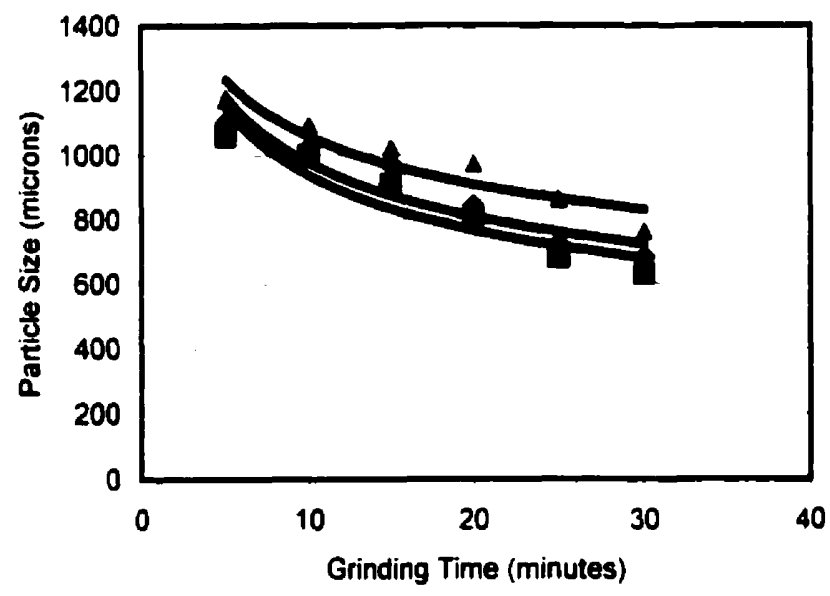

$350 \mathrm{~g}$

a $750 \mathrm{~g}$

- 2009 